\title{
THE STRUCTURE EQUATIONS OF A COMPLEX FINSLER MANIFOLD*
}

\author{
A. SPIRO ${ }^{\dagger}$
}

A mio padre

\begin{abstract}
For a strongly pseudo-convex complex Finsler manifold $M$, a bundle $U_{F}(M)$ of adapted unitary frames is canonically defined. A non-linear Hermitian connection on $U_{F}(M)$, invariant under local biholomorphic isometries, is given and it proved to be unique. By means of such connection, an absolute parallelism on $U_{F}(M)$ is determined and a new set of structure functions which generate all the isometric invariants of a Finsler metric is obtained.

A pseudo-convex complex Finsler manifolds $M$, which admits a totally geodesic complex curve with a given constant holomorphic sectional curvature through any point and any direction, is called E-manifold. Main examples of E-manifolds are the smoothly bounded, strictly convex domains in $\mathbb{C}^{n}$, endowed with the Kobayashi metric. A complete characterization of E-manifolds, using the previously defined structure functions, is given and a smaller set of generating functions for the isometric invariants of E-manifolds is determined.
\end{abstract}

1. Introduction. The main purpose of this paper is to give a complete set of invariants, which characterize a strongly pseudoconvex complex Finsler metric up to local biholomorphic isometries. Several properties of these invariants are immediately related with the intrinsic geometry of the Kobayashi metric of the smoothly bounded, strongly convex domains in $\mathbb{C}^{n}$.

Let $M$ be a complex manifold and $J$ its complex structure. The well-known infinitesimal Kobayashi pseudo-distance $k_{M}$ on $\tilde{T} M=T M \backslash$ zero section can be defined as follows ([Ko]): for any $x \in M$ and any $0 \neq v \in T_{x} M$, let $\mathcal{A}_{v}$ the set of all $r \in \mathbb{R}^{+}$such that there exists a holomorphic map $f: \Delta_{r} \rightarrow M$ from $\Delta_{r}=\{|z|<r\} \subset$ $\mathbb{C}$ into $M$ with $f(0)=x$ and $f_{*}\left(\frac{\partial}{\partial \zeta}\right) \in \mathbb{C} v$. Then

$$
k_{M}(v)=\inf _{r \in \mathcal{A}(v)} \frac{1}{r} .
$$

We consider the following class of complex manifolds.

Definition 1.1. A complex manifold $(M, J)$ is a Lempert manifold if

(1) the infinitesimal Kobayashi pseudo-distance $k_{M}$ is a strongly pseudoconvex Finsler metric, that is:

a) it is a smooth function on $\tilde{T} M$ with values in $\mathbb{R}^{+}$;

b) $k_{M}(\lambda v)=|\lambda| k_{M}(v)$ for any $\lambda \in \mathbb{C}^{*}$ and $v \in \tilde{T} M$;

c) at any point $x \in M$ the hypersurface $S_{x}=\left\{v \in T_{x} M: k_{M}(v)=1\right\}$ is strongly pseudoconvex in $T_{x} M$;

(2) for any non-vanishing complex vector $w \in T_{x}^{\mathbb{C}} M \subset T^{\mathbb{C}} M$, there exists a complex curve $\gamma_{w}: U \subset \mathbb{C} \rightarrow M$, such that $\gamma_{w}(0)=x, \gamma_{w}^{\prime}(0)=w$ and $\gamma(U)$ is a totally geodesic submanifold of $M$;

(3) the metric, which is induced by $k_{M}$ on the totally geodesic complex curve $\gamma_{w}(U)$, is Kähler and with constant holomorphic curvature equal to -4 ;

(4) the (finite) Kobayashi distance $d_{M}$, determined by $k_{M}$, is complete and the exponential map exp : $T_{x} M \rightarrow M$ is a diffeomorphism for any $x \in M$.

* Received January 17, 2000; accepted for publication May 26, 2000.

† Dipartimento di Matematica e Fisica, Università di Camerino, 62032 CAMERINO (Macerata), ITALY (spiro@campus.unicam.it). 
An immediate interest for Lempert manifolds comes from the well-known results of L. Lempert on the Kobayashi metric of strongly convex domains in $\mathbb{C}^{n}$ ([Le], [Le1]). Some of his results can be stated as follows.

TheOREM 1.2. [Le] If $M$ is a smoothly bounded, strongly convex domain in $\mathbb{C}^{n}$, then $M$ is a Lempert manifold.

Since the convexity of a domain is not a biholomorphic invariant property, Theorem 1.2 motivates the following question:

Are there some invariant properties of $k_{M}$ (to be added to (1) - (4) of Def. 1.1), which characterize the manifolds that are biholomorphic to a smoothly bounded, strongly convex domain in $\mathbb{C}^{n}$ ?

Some encouraging results have been obtained by various authors (e.g. [Fa], $[\mathrm{Pa}]$, [Le2], [BD], [AP], [AP1]). In particular, we would like to mention the following theorem by J. J. Faran (see also [Pa]).

THEOREM 1.3. [Fa] $(M, J)$ is a Lempert manifold if and only if it admits a strongly pseudoconvex Finsler metric $F$, which verifies (2), (3) and (4) of Definition 1.1. In this case $F$ coincides with the Kobayashi metric $k_{M}$.

Faran's Theorem has been improved by M. Abate and G. Patrizio in [AP] in the following sense: they proved that if $(M, J)$ has a strongly pseudoconvex Finsler metric $F$, then it admits a natural non-linear Finsler connection and if the corresponding torsion and curvature verify pointwise a certain set of conditions, then $(M, J)$ satisfies (2), (3) and (4) of Def. 1.1 and hence it is a Lempert manifold.

By Faran's theorem, our previous question has positive answer if and only if there exist some conditions, which are necessary and sufficient for the existence of a biholomorphism between a Finsler manifold $M$, verifying (2), (3) and (4) of Def. 2.1, and a strongly convex domain $D \subset \mathbb{C}^{n}$.

The general problem of determining necessary and sufficient conditions for the existence of a (local) isomorphism between two geometric structures is usually called the (local) equivalence problem for those structures. In this paper we give a new solution to the local equivalence problem for strongly pseudoconvex Finsler metrics and, by means of this solution, we obtain a new complete set of invariant functions which determine the complex Finsler metrics up to local biholomorphic isometries. We also use these invariants to give a new characterization of Lempert manifolds.

Here are the contents of the paper. In $\S 2$ we recall and prove some preliminary properties of complex Finsler metrics.

In $\S 3$ we introduce the concept of adapted unitary frames of a complex manifold $(M, J)$ with a strongly pseudoconvex Finsler metric $F$. The bundle $U_{F}(M)$ of all adapted unitary frames turns out to be a subbundle of the complex linear frame bundle $L^{\mathbb{C}}(M)$, but in general it is not a principal subbundle; this is the case if and only if there exists an Hermitian metric $g$ so that $F(v)=\sqrt{g(v, v)}$ for all $0 \neq v \in T M$.

We also use the following terminology: any distribution which is complementary to the vertical distribution and of dimension equal to $\operatorname{dim} M$ is named a non-linear connection on $U_{F}(M)$. We say that a non-linear connection is of Hermitian type if it is invariant w.r.t. the complex structure $\hat{J}$ of $L^{\mathbb{C}}(M)$.

The main result of $\S 3$ is the following (Theorem 3.9). 
TheOREM 1.4. Let $(M, J, F)$ be a strongly pseudoconvex Finsler manifold. Then the unitary frame bundle $U_{F}(M)$ has a unique non-linear connection of Hermitian type. This connection is invariant under any biholomorphic isometry of $(M, J, F)$.

This non-linear Hermitian connection on $U_{F}(M)$ defines a non-linear covariant derivation for vector fields of $M$ which is invariant under any biholomorphic isometry.

In $\S 4$ we show that any fiber $\mathbb{V}_{x}=\pi^{-1}(x)$ of $U_{F}(M)$ is identifiable with the adapted frame bundle of a pseudo-hermitian structure on the Finsler sphere $S_{x}$. Using the Webster connection for pseudo-hermitian structures (see [We]), we define an invariant absolute parallelism on each fiber $\mathbb{V}_{x}$, i.e. a set of vertical vector fields on $\mathbb{V}_{x}$, which at all points span $T_{u} \mathbb{V}_{x}$ and which is invariant under the automorphism of the pseudo-hermitian structure of $S_{x}$.

Using this absolute parallelism on the fibers and the non-linear Hermitian connection $\mathcal{H}$ of $U_{F}(M)$, we obtain an absolute parallelism $\sigma$ on $U_{F}(M)$ which verifies the following crucial property: the (local) biholomorphic isometries of $(M, J, F)$ are in 1-1 correspondence with the (local) diffeomorphisms of $U_{F}(M)$ which preserve $\sigma$.

By Kobayashi's theorem on the automorphisms of absolute parallelisms ([Kol]), we immediately obtain the following result (Proposition 4.6):

THEOREM 1.5. Let $(M, J)$ be a complex manifold of complex dimension $n$ and $F$ a strongly pseudoconvex Finsler metric on $(M, J)$.

The group of biholomorphic isometries $\operatorname{Iso}_{F}(M, J)$ is a Lie group of dimension less or equal to $n^{2}+2 n$. Moreover $\operatorname{dim}_{\mathbb{R}} I \operatorname{Iso}_{F}(M, J)=n^{2}+2 n$ if and only if $F$ is equal to $F(v)=\sqrt{g(v, v)}$ for some Kähler metric $g$ of constant holomorphic sectional curvature and $(M, J, g)$ is a simply connected complex space form, i.e. $\mathbb{C} P^{n}, \mathbb{C}^{n}$ or the unit ball $B^{n} \subset \mathbb{C}^{n}$, endowed with a Fubini-Study, flat or Poincarè-Bergmann metric, respectively

In $\S 5$ we determine the Lie brackets of all possible pairs of vector fields of the absolute parallelism $\sigma$ of $U_{F}(M)$. By Cartan-Sternberg theorem the components of these Lie brackets w.r.t. the vectors of the absolute parallelism generate a complete set of invariant functions for the Finsler manifold $(M, J, F)$ (see Proposition 4.6 and Theorem A1). At the end of $\S 5$, we also give the so-called structure equations of the Finsler manifold of $(M, J, F)$, i.e. the equations that are verified by the 1 -forms on $U_{F}(M)$ which are dual to the vector fields of the absolute parallelism.

At last, in $\S 6$, we determine the Euler-Lagrange equations for the geodesics of a complex Finsler manifold. We recall the definition of complex geodesics (see [Ve] and [AP]) and we find necessary and sufficient conditions for a complex Finsler manifold to be of constant holomorphic sectional curvature and with a complex geodesic through any point and any direction. We call such manifolds $E$-manifolds. Notice that the Lempert manifolds are complete E-manifolds with holomorphic sectional curvature equal to -4 .

For the E-manifold, we also prove that the torsion and the curvature can be expressed in terms of the other structure functions of the absolute parallelism on $U_{F}(M)$ and hence that these structure functions are the actual generators for the invariants of E-manifolds (see Theorem 6.9).

We have to mention that an alternative solution to the equivalence problem has been given by J. J. Faran in [Fa]. He determines another set of invariant functions, by pursuing the steps of a general algorithmic procedure: it is our personal opinion that, 
by this reason, it is quite cumbersome to obtain simple geometric interpretations for the invariant functions introduced by Faran.

As final remark, we want to point out that our non-linear covariant derivation on complex Finsler manifolds is strictly related (but different) with the non-linear covariant derivation invented by $\mathrm{S}$. Kobayashi in [Ko2]. In a forthcoming paper, we will discuss this relation and we will show how to use the components of the curvature and torsion tensors in order to determine the set of invariant functions given in $\S 5$.

\section{Preliminaries.}

2.1. Notation and basic definitions. In all this paper, we use greek letters $\alpha, \beta$, etc. for indices related to holomorphic vectors, barred greek letters $\bar{\alpha}, \bar{\beta}$, etc. for indices related to the conjugated vectors and latin indices $i, j, k$, etc. to denote real vectors.

We denote by $\left\{\epsilon_{0}, \epsilon_{1}, \ldots, \epsilon_{2 n-1}\right\}$ the standard real basis of $V=\mathbb{R}^{2 n}=\mathbb{C}^{n} ; J_{o}$ is the complex structure of $\mathbb{C}^{n}$. The standard basis is ordered so that $J_{o}\left(\epsilon_{2 i}\right)=\epsilon_{2 i+1}$ for any $i=0, \ldots, n$. We set $\varepsilon_{\alpha}=\frac{1}{2}\left(\epsilon_{2 \alpha}-\sqrt{-1} \epsilon_{2 \alpha+1}\right), \alpha=0, \ldots, n-1$, and $\varepsilon_{\bar{\alpha}}=\overline{\varepsilon_{\alpha}}$. We also use the notation $\left\{\epsilon^{i}\right\},\left\{\varepsilon^{\alpha}\right\}$ and $\left\{\varepsilon^{\bar{\alpha}}\right\}$ for the dual bases of $\left\{\epsilon_{i}\right\},\left\{\varepsilon_{\alpha}\right\}$ and $\left\{\varepsilon_{\bar{\alpha}}\right\}$, respectively.

$<,>$ is the standard Hermitian product of $V=\mathbb{C}^{n}$.

$W$ denotes the subspace

$$
W=\operatorname{span}_{\mathbb{C}}\left\{\varepsilon_{1}, \ldots, \varepsilon_{n-1}\right\}=\mathbb{C}^{n-1} .
$$

$(M, J)$ is always a complex manifold with complex structure $J$ and complex dimension $n$. We let $\tilde{T} M=T M \backslash\{$ zero section $\}$ and $\mathbb{P} T M=\tilde{T} M / \mathbb{C}^{*}$.

For any $v \in T_{x} M, \imath_{v}: T_{x} M \stackrel{\sim}{\longrightarrow} T_{v}\left(T_{x} M\right)$ is the natural isomorphism between $T_{x} M$ and $T_{v}\left(T_{x} M\right)$. Using the maps $\imath_{v}$, any vector $w \in T_{v_{o}}\left(T_{x} M\right) \subset T\left(T_{x} M\right)$ extends to a vector field $X^{(w)}$ on $T_{x} M$ by letting $\left.X^{(w)}\right|_{v}=\imath_{v} \circ \imath_{v_{o}}^{-1}(w)$. We call $X^{(w)}$ the trivial extension of $w$.

For any $v \in T_{x} M, J$ denotes both the complex structure on $T_{x} M$ and on $T_{v}\left(T_{x} M\right)$. The vectors $v^{10}$ and $v^{01}$ are the holomorphic and anti-holomorphic components of $v$ w.r.t. $J$

$$
v^{10}=\frac{1}{2}(v-\sqrt{-1} J v), \quad v^{01}=\frac{1}{2}(v+\sqrt{-1} J v) .
$$

The dilatation field $D$ (also called Euler vector field) is the vector field on $T M$ defined as

$$
\left.D\right|_{v}=\imath_{v}(v)
$$

A linear frame at a point $x$ of $M$ is an $\mathbb{R}$-linear isomorphism $u: \mathbb{R}^{2 n} \rightarrow T_{x} M$. A complex linear frame at a point $x$ is a $\mathbb{C}$-linear isomorphism $u: \mathbb{C}^{n} \rightarrow T_{x} M$. We always identify a linear frame $u$ with the corresponding basis $\left\{f_{i}\right\}$ in $T_{x} M$ where

$$
f_{i}=u\left(\epsilon_{i}\right) \in T_{x} M .
$$

If $u$ is complex, we denote by $u^{10}$ the corresponding holomorphic basis, that is

$$
u^{10}=\left\{e_{\alpha}=u\left(\varepsilon_{\alpha}\right)=\frac{1}{2}\left(f_{2 \alpha}-\sqrt{-1} f_{2 \alpha+1}\right)\right\} .
$$

For any linear frame $u$ on $T_{x} M$, the point $x=\pi(u)$ is called base point of $u$.

An absolute parallelism is a set of vector fields $\left\{X_{1}, \ldots, X_{2 n}\right\}$ which are linearly independent at all points and, hence, constitute a smooth field of frames on $M$. 
The collection of all linear frames on $M$ is denoted by $L(M)$; recall that it is a $G L_{2 n}(\mathbb{R})$-bundle w.r.t. the projection map $\pi$. The collection of all complex linear frames is denoted by $L^{\mathbb{C}}(M)$; it is a principal $G L_{n}(\mathbb{C})$-subbundle of $L(M)$.

It is well-known that $L^{\mathbb{C}}(M)$ admits a unique complex structure $\hat{J}$ which verifies the following two conditions:

a) the restriction of $\hat{J}$ to the vertical subspaces of $L^{\mathbb{C}}(M)$ coincides with the complex structure of $G L_{n}(\mathbb{C})$;

b) the projection $\pi: L^{\mathbb{C}}(M) \rightarrow M$ is holomorphic.

We call $\hat{J}$ the standard complex structure of $L^{\mathbb{C}}(M)$.

For any subbundle $P \subset L(M)$, we denote by $\theta$ its tautological 1 -form, which is defined as follows. For any frame $u=\left\{f_{i}\right\} \in P \subset L(M)$ and any vector $X \in T_{u} P$, the projected vector $\pi_{*}(X)$ can be written as $\pi_{*}(X)=\sum_{i=0}^{2 n-1} \theta_{u}^{i}(X) f_{i}$ for some numbers $\theta_{u}^{i}(X)$. The tautological 1 -form $\theta$ is the $\mathbb{R}^{2 n}$-valued 1 -form

$$
\theta_{u}(X)=\sum_{i=0}^{2 n-1} \theta_{u}^{i}(X) \cdot \epsilon_{i} .
$$

If $P$ is a subbundle of $L^{\mathbb{C}}(M)$, any vector $X \in T_{u} P$ admits the decomposition $X=$ $X^{10}+X^{01}=X^{10}+\overline{X^{10}}$, where $X^{10}$ is the holomorphic part of $X$ w.r.t. $\hat{J}$. We denote by $\theta_{u}^{\alpha}\left(X^{10}\right)$ and $\theta_{u}^{\bar{\alpha}}\left(X^{01}\right)$ the components of $\pi_{*}\left(X^{10}\right)$ and $\pi_{*}\left(X^{01}\right)$ w.r.t. the holomorphic and anti-holomorphic frame $u^{10}$ and $u^{01}$, respectively. In this way two sets of $\mathbb{C}$-valued 1 -forms $\theta^{\alpha}$ and $\theta^{\bar{\alpha}}$ are defined at all points of $P$. They are called holomorphic and antiholomorphic components of the tautological 1-form $\theta$.

Finally, for any $A \in \mathfrak{g l}_{2 \mathfrak{n}}(\mathbb{R})$, we denote by $A^{*}$ the associated fundamental vector field, that is the vector field on $L(M)$ whose flow is

$$
\Phi_{t}^{A^{*}}(u)=u \circ \exp (t A) .
$$

Since $G L_{n}(\mathbb{C})$ acts freely and transitively on the fibers of $L^{\mathbb{C}}(M)$, the fundamental vector fields span any vertical subspace $\mathcal{V}_{u} \subset T_{u} L^{\mathbb{C}}(M)$. Therefore if $P$ is a subbundle of $L^{\mathbb{C}}(M)$ (not necessarily a principal subbundle), we may consider the subspace $\mathfrak{g}_{u} \subset$ $\mathfrak{g l} \mathfrak{l}_{\mathfrak{n}}(\mathbb{C})$

$$
\mathfrak{g}_{u}=\left\{A \in \mathfrak{g l}_{\mathfrak{n}}(\mathbb{C}), A_{u}^{*} \in T_{u} P\right\} .
$$

We call $\mathfrak{g}_{u}$ the algebraic vertical subspace of $P$ at the point $u$. Notice that $P$ is a principal subbundle if and only if $\mathfrak{g}_{u}$ is a subalgebra of $\mathfrak{g l}_{\mathfrak{n}}(\mathbb{C})$ independent on $u \in P$. In this case $\mathfrak{g}_{u}=\mathfrak{g}=\operatorname{Lie}(G)$, where $G$ is the structure group of $P$.

\subsection{First properties of complex Finsler manifolds.}

Definition 2.1. A complex Finsler metric on $(M, J)$ is a continuous function

$$
F: T M \longrightarrow \mathbb{R}^{+}
$$

satisfying the following properties:

i) $F$ is smooth on $\tilde{T} M$;

ii) $F(u)>0$, for all $u \in \tilde{T} M$;

iii) $F(\lambda u)=|\lambda| F(u)$ for all $u \in \tilde{T} M$ and any $\lambda \in \mathbb{C}$.

A complex Finsler manifold is a complex manifold $(M, J)$ endowed with a complex Finsler metric $F$. 
A (local) biholomorphism $f: M \rightarrow N$ between two complex Finsler manifolds $(M, J, F)$ and $\left(N, J^{\prime}, F^{\prime}\right)$ is called (local) biholomorphic isometry if $F^{\prime}\left(f_{*} v\right)=F(v)$, for any $v \in T M$.

Note that any complex Finsler metric $F$ is in particular a real Finsler metric (see e.g. [Ca], [Ch], [Ch1], [BC], [AP], [Sp]).

For any complex Finsler manifold $(M, J, F)$, the Finsler pseudo-sphere at a point $x$ is the hypersurface

$$
S_{x}=\left\{v \in T_{x} M: F(v)=1\right\} \subset T_{x} M .
$$

We say that $F$ is associated with the Hermitian metric $g$ if for any $v \in T M$

$$
F(v)=\sqrt{g(v, v)} .
$$

If this is the case, for any $x \in M$ the hermitian metric $g_{x}$ is recovered from $F$ by

$$
g_{x}(v, w)=\frac{1}{2} \mathrm{~h}_{v_{o}}(v, w),
$$

where $h$ is the quadratic form defined in the following formula (2.6) and $v_{o}$ is any non zero vector of $T_{x} M$. Note that if $\operatorname{dim}_{\mathbb{C}} M=1$, then any complex Finsler metric is associated with a Kähler metric $g$.

The quadratic form $h$, the cubic form $H$ and the quartic form $\mathbf{H}$ of a complex Finsler metric $F$ are the following multilinear forms on $T(\tilde{T} M)$. Let $X, Y, Z, W \in$ $T_{v}\left(T_{x} M\right)$ and $\hat{X}, \hat{Y}, \hat{Z}$ and $\hat{W}$ be their trivial extensions. Then we set

$$
\begin{gathered}
\mathrm{h}_{v}(X, Y)=\left.\hat{X}\left[\hat{Y}\left(F^{2}\right)\right]\right|_{v} ; \quad \mathrm{H}_{v}(X, Y, Z)=\left.\hat{X}\left[\hat{Y}\left[\hat{Z}\left(F^{2}\right)\right]\right]\right|_{v} ; \\
\mathbf{H}_{v}(X, Y, Z, W)=\left.\hat{X}\left[\hat{Y}\left[\hat{Z}\left[\hat{W}\left(F^{2}\right)\right]\right]\right]\right|_{v} .
\end{gathered}
$$

Since any set of trivial extensions commute, it is immediate to realize that $\mathrm{h}, \mathrm{H}$ and $\mathbf{H}$ are multilinear and totally symmetric in their arguments.

In all the following, for any $v, w, z, t, y \in T_{x} M$, we will use the simplified notation $\mathrm{h}_{v}(w, z), \mathrm{H}_{v}(w, z, t)$ and $\mathbf{H}_{v}(w, z, t, y)$ in place of $\mathrm{h}_{v}\left(\imath_{v}(w), \imath_{v}(z)\right), \mathrm{H}_{v}\left(\imath_{v}(w)\right.$, $\left.\imath_{v}(z), \imath_{v}(t)\right)$ and $\mathbf{H}_{v}\left(\imath_{v}(w), \imath_{v}(z), \imath_{v}(t), \imath_{v}(y)\right)$, respectively.

The quadratic form $\widetilde{h}$, the cubic form $\widetilde{H}$ and the quartic form $\widetilde{\mathbf{H}}$ of a Finsler pseudo-sphere $S_{x} \subset T_{x} M$ are the the restrictions on $T\left(S_{x}\right)$ of h, $\mathrm{H}$ and $\mathbf{H}$. Note that, since a Finsler pseudo-sphere is a level set of $F^{2}$, it follows that $\widetilde{\mathrm{h}}_{v}$ is equal to

$$
\widetilde{\mathrm{h}}_{v}(X, Y)=\hat{X}^{\prime}\left(\hat{Y}^{\prime}\left(F^{2}\right)\right)
$$

where $\hat{X}^{\prime}, \hat{Y}^{\prime}$ are two arbitrary vector fields, which are tangent to $S_{x}$ and which coincide with $X$ and $Y$ at $v \in S_{x}$. A similar result holds for $\widetilde{\mathrm{H}}$ and $\widetilde{\mathbf{H}}$.

Since $\rho_{x}=\left.\left(F^{2}-1\right)\right|_{T_{x} M}$ is a defining function for the Finsler pseudo-sphere $S_{x}$, we have the following immediate Lemma .

Lemma 2.2. Let $\mathcal{D}_{v} \subset T_{v}\left(S_{x}\right)$ be the maximal $J$-invariant subspace of $T_{v}\left(S_{x}\right)$ of a Finsler pseudo-sphere $S_{x}$ and let $\mathcal{D}_{v}^{\mathbb{C}}=\mathcal{D}_{v}^{10}+\mathcal{D}_{v}^{01}$ the corresponding decomposition into holomorphic and anti-holomorphic subspaces. Let also $\mathcal{L}_{v}$ the Levi form of $S_{x} \subset T_{x} M$ given by $\rho_{x}$ (for the definition, see e.g. (3.1) in §3). Then for any $X^{10}, Y^{10} \in \mathcal{D}_{v}^{10}$,

$$
\mathcal{L}_{v}\left(X^{10}, Y^{10}\right)=\widetilde{h}_{v}\left(X^{10}, \overline{Y^{10}}\right) .
$$


Definition 2.3. A complex Finsler metric $F$ is called strongly pseudoconvex (resp. Levi non-degenerate) if for any $x \in M$ the Levi form of the Finsler pseudosphere $S_{x}$ is positive definite (resp. non-degenerate) at all points.

Note that if a complex Finsler metric $F$ is strictly convex as real Finsler metric (for the definition, see f.i. [Ch], [AP] or [Sp]), then it is also strongly pseudoconvex. The converse is obviously not true.

The following two Lemmata give some basic properties of $\mathrm{h}, \mathrm{H}$ and $\mathbf{H}$.

Lemma 2.4. Let $(M, J, F)$ be a complex Finsler manifold. For any $0 \neq v \in T_{x} M$ consider the trivial extension $\hat{v}$ of the vector $D_{v}=\imath_{v}(v)$ and let $X, Y, Z, X_{i} \in$ $T\left(T_{x} M\right), i=1, \ldots, k$, some trivially extended vector fields. Then

a) $D\left(F^{2}\right)_{v}=2 F_{v}^{2}$ and $(J D)\left(F^{2}\right)_{v}=0$;

b) $\left.D\left(X_{1}\left(X_{2}\left(\ldots X_{k}\left(F^{2}\right) \ldots\right)\right)\right)\right|_{v}=\left.(2-k) X_{1}\left(X_{2}\left(\ldots X_{k}\left(F^{2}\right) \ldots\right)\right)\right|_{v}$;

c) $\left.J X_{1}\left(X_{2}\left(\ldots X_{k}\left(F^{2}\right) \ldots\right)\right)\right|_{v}+\left.X_{1}\left(J X_{2}\left(\ldots X_{k}\left(F^{2}\right) \ldots\right)\right)\right|_{v}+\ldots$

$\cdots+\left.X_{1}\left(X_{2}\left(\ldots J X_{k}\left(F^{2}\right) \ldots\right)\right)\right|_{v}+\left.J D\left(X_{1}\left(X_{2}\left(\ldots X_{k}\left(F^{2}\right) \ldots\right)\right)\right)\right|_{v}=0$

d) $D^{10}\left(F^{2}\right)=D^{01}\left(F^{2}\right)=F^{2}$ and

$$
h_{v}\left(X^{10}, \hat{v}^{10}\right)=0, \quad h_{v}\left(X^{10}, \hat{v}^{01}\right)=X^{10}\left(F^{2}\right)_{v} ;
$$

e)

(2.10) $H_{v}\left(X^{10}, Y^{01}, v^{10}\right)=H_{v}\left(X^{10}, Y^{01}, v^{01}\right)=0$,

(2.11) $H_{v}\left(X^{10}, Y^{10}, v^{10}\right)=-h_{v}\left(X^{10}, Y^{10}\right), H_{v}\left(X^{10}, Y^{10}, v^{01}\right)=h_{v}\left(X^{10}, Y^{10}\right)$,

(2.12) $\mathbf{H}_{v}\left(\hat{v}^{01}, X^{10}, Y^{10}, Z^{01}\right)=0, \quad \mathbf{H}_{v}\left(\hat{v}^{10}, X^{10}, Y^{01}, Z^{01}\right)=0$,

(2.13) $\mathbf{H}_{v}\left(\hat{v}^{10}, X^{10}, Y^{10}, Z^{01}\right)=-H_{v}\left(X^{10}, Y^{10}, Z^{01}\right)$,

(2.14) $\mathbf{H}_{v}\left(\hat{v}^{01}, X^{10}, Y^{01}, Z^{01}\right)=-H\left(X^{10}, Y^{01}, Z^{01}\right)$.

Proof. Consider on $T_{x} M$ the flows

$$
\Phi_{t}, \Psi_{t}: T_{x} M \rightarrow T_{x} M, \quad \Phi_{t}(v)=e^{t} \cdot v, \quad \Psi_{t}(v)=e^{t J} \cdot v .
$$

Definition 2.1 (iii) is equivalent to

$$
\left(F^{2} \circ \Phi_{t}\right)(v)=e^{2 t} F^{2}(v), \quad\left(F^{2} \circ \Psi_{t}\right)(v)=F^{2}(v)
$$

for any $v \in \tilde{T} M$ and any $t \in \mathbb{R}$. If we identify any vector $X \in T_{v}\left(T_{x} M\right)$ with the corresponding element in $T_{\Phi_{t}(v)}\left(T_{x} M\right)$ and $T_{\Psi_{t}(v)}\left(T_{x} M\right)$, the differentials $\Phi_{t *}$ and $\Psi_{t *}$ can be written as

$$
\left.\Phi_{t *}\right|_{v}(X)=e^{t} \cdot X,\left.\quad \Psi_{t *}\right|_{v}(X)=e^{t J} \cdot X .
$$

Therefore for any trivially extended vector fields $X_{i} \in T\left(T_{x} M\right), i=1, \ldots k$,

$$
\begin{aligned}
& e^{k t} \cdot\left[\left.X_{1}\left(X_{2}\left(\ldots\left(X_{k}\left(F^{2}\right)\right) \ldots\right)\right)\right|_{e^{t} v}\right] \\
= & {\left.\left[\Phi_{t *}\left(X_{1}\right)\left[\Phi_{t *}\left(X_{2}\right)\left[\ldots\left[\Phi_{t *}\left(X_{k}\right)\left[F^{2}\right]\right] \ldots\right]\right]\right]\right|_{\Phi_{t}(v)} } \\
= & \left.e^{2 t}\left(X_{1}\left(X_{2}\left(\ldots X_{k}\left(F^{2}\right)\right)\right)\right)\right|_{v}
\end{aligned}
$$




$$
\begin{aligned}
& \left.e^{t J} \cdot X_{1}\left(e^{t J} \cdot X_{2}\left(\ldots\left(e^{t J} \cdot X_{k}\left(F^{2}\right)\right) \ldots\right)\right)\right|_{\exp (t J) v} \\
= & \left.\Psi_{t *}\left(X_{1}\right)\left[\Psi_{t *}\left(X_{2}\right)\left[\ldots\left[\Psi_{t *}\left(X_{k}\right)\left[F^{2}\right]\right] \ldots\right]\right]\right|_{\Psi_{t}(v)} \\
= & \left.X_{1}\left(X_{2}\left(\ldots\left(X_{k}\left(F^{2}\right)\right) \ldots\right)\right)\right|_{v} .
\end{aligned}
$$

Taking the derivative at $t=0$ of (2.16), (2.18) and (2.19), one immediately obtains a), b) and c).

d) follows from a), b).

To prove e), observe that b) and c) imply that

$$
\begin{gathered}
\mathrm{H}_{v}(V, W, v)=\hat{v}\left(V\left(W\left(F^{2}\right)\right)\right)_{v}=D\left(V\left(W\left(F^{2}\right)\right)\right)_{v}=(2-2) V\left(W\left(F^{2}\right)\right)_{v}=0, \\
\hat{v}\left(V\left(W\left(Z\left(F^{2}\right)\right)\right)\right)_{v}=(2-3) \mathrm{H}_{v}(V, W, Z)=-\mathrm{H}_{v}(V, W, Z),
\end{gathered}
$$

and

$$
\begin{gathered}
\mathrm{H}_{v}(V, W, J v)=J D\left(V\left(W\left(F^{2}\right)\right)\right)_{v}=-\mathrm{h}_{v}(J V, W)-\mathrm{h}_{v}(V, J W), \\
J D\left(V\left(W\left(Z\left(F^{2}\right)\right)\right)\right)_{v}=-\mathrm{H}_{v}(J V, W, Z)-\mathrm{H}_{v}(V, J W, Z)-\mathrm{H}_{v}(V, W, J Z) .
\end{gathered}
$$

From these identities and some straightforward computations (2.10) - (2.14) follow.

Lemma 2.5. Let $(M, J, F)$ be a strongly pseudo-convex Finsler manifold. Then $F$ is associated with an Hermitian metric $g$ if and only if one of the following two equivalent conditions are satisfied:

i) the cubic form $H$ vanishes identically;

ii) for any point $x \in M$, any vector $0 \neq v \in T_{x} M$ and any $X, Y \in T_{v}\left(T_{x} M\right)$

$$
h_{v}\left(X^{10}, Y^{10}\right)=0 \text {. }
$$

Proof. If $F$ is associated with an Hermitian metric, then (ii) is clearly satisfied. Moreover, if (ii) holds, for any three vectors $0 \neq X, Y, Z \in T_{v}\left(T_{x} M\right)$, with trivial extensions $\hat{X}, \hat{Y}$ and $\hat{Z}$, we get

$$
\begin{aligned}
& \mathrm{H}_{v}\left(X^{10}, Y^{10}, Z^{10}\right)=\left.\hat{Z}^{10}\left(\mathrm{~h}\left(X^{10}, Y^{10}\right)\right)\right|_{v}=0, \\
& \mathrm{H}_{v}\left(X^{10}, Y^{10}, Z^{01}\right)=\left.\hat{Z}^{01}\left(\mathrm{~h}\left(X^{10}, Y^{10}\right)\right)\right|_{v}=0,
\end{aligned}
$$

and this implies (i). So, in order to conclude, we just need to show that (i) implies that $F$ is associated with an Hermitian metric.

Note that if (i) holds, for any two trivially extended vector fields $\hat{X}$ and $\hat{Y}$, the value of $\mathrm{h}_{v}(\hat{X}, \hat{Y})$ is independent of $v$. Moreover, from $(2.11), \mathrm{h}_{v}\left(\hat{X}^{10}, \hat{Y}^{10}\right)=$ $\mathrm{H}_{v}\left(\hat{X}^{10}, \hat{Y}^{10}, \hat{v}^{01}\right)=0$ and hence

$$
\mathrm{h}_{v}(\hat{X}, \hat{Y})=\mathrm{h}_{v}\left(\hat{X}^{10}, \hat{Y}^{01}\right)+\mathrm{h}_{v}\left(\hat{X}^{01}, \hat{Y}^{10}\right)
$$

So the quadratic form $g_{x}$ defined by (2.5) is an Hermitian metric on $T_{x} M$ and, by Lemma $2.4 \mathrm{~d}), g_{x}(v, v)=\mathrm{h}_{v}\left(v^{10}, v^{01}\right)=D^{10}\left(F^{2}\right)_{v}=F_{v}^{2}$.

From this point on, if the opposite is not stated, by complex Finsler metric and complex Finsler manifold we will mean strongly pseudoconvex complex Finsler metric and strongly pseudoconvex complex Finsler manifold, respectively. fold.

3. The non-linear Hermitian connection of a complex Finsler mani- 


\subsection{The $\mathbf{H}$-sphere bundle of a complex Finsler manifold.}

Definition 3.1. An Hermitianized sphere bundle on $(M, J)$ (or, more shortly, $H$-sphere bundle) is a pair $(S M, \rho)$ where:

a) $S M \subset T M$ is a smooth subbundle;

b) each fiber $S_{x} \subset T_{x} M$ is a star shaped strongly pseudoconvex hypersurface of $\left(T_{x} M, J\right)$, diffeomorphic to a sphere;

c) $\rho$ is a smooth real function on $\tilde{T} M$ such that $S M=\{v \in \tilde{T} M: \rho(v)=0\}$. An H-sphere bundle $(S M, \rho)$ is called circular if $S M$ is invariant with respect to the linear group of transformations $T^{1}=\left\{e^{t J}, t \in \mathbb{R}\right\}$ and $\rho$ is $T^{1}$-invariant.

Two H-spheres bundles $(S M, \rho),\left(S M^{\prime}, \rho^{\prime}\right)$ over $(M, J)$ and $\left(M^{\prime}, J^{\prime}\right)$, respectively, are biholomorphically isometric if there exists a biholomorphism $f: M \rightarrow M^{\prime}$, such that $\rho=\left.\rho^{\prime} \circ f_{*}\right|_{\tilde{T} M}$.

The main examples of $\mathrm{H}$-sphere bundles are the Finsler sphere bundles.

Definition 3.2. The $H$-sphere bundle of a complex Finsler manifold $(M, J, F)$ is the pair $\left(S^{F} M, \rho_{F}\right)$, where $S^{F} M$ is the bundle of the Finsler spheres in $\tilde{T} M$ and $\rho_{F}=F^{2}-1$.

Notice that $\left(S^{F} M, \rho_{F}\right)$ is always circular. Moreover it is clear that two Finsler manifolds are biholomorphically isometric if and only if the corresponding $\mathrm{H}$-sphere bundles are biholomorphically isometric.

Remark 3.3. Let $S_{x} \subset T_{x} M$ be a sphere of a circular $\mathrm{H}$-sphere bundle $(S M, \rho)$ and $\rho_{x}$ the restriction $\rho_{x}=\left.\rho\right|_{T_{x} M}$, so that $S_{x}=\left\{\rho_{x}(v)=0\right\}$. Let also $\mathcal{D}_{x} \subset T S_{x}$ be the family of the maximal $J$-invariant tangent spaces of $S_{x}$ and $\mathcal{L}_{x}$ the Levi form of $S_{x}$, i.e. the collection of the Hermitian forms on the spaces $\left.\mathcal{D}_{x}\right|_{v} \subset T_{v} S_{x}, v \in S_{x}$, defined by

$$
\left.\mathcal{L}_{x}(X, Y)\right|_{v}=\left.\hat{X}\left(J \hat{Y}\left(\rho_{x}\right)\right)\right|_{v},
$$

where in the right hand side $\hat{X}$ and $\hat{Y}$ denote the trivial extensions of the vectors $X,\left.Y \in \mathcal{D}_{x}\right|_{v}$.

By definitions, $\mathcal{D}_{x}$ and $\mathcal{L}_{x}$ are $T^{1}$-invariant and each space $\left.\mathcal{D}_{x}\right|_{v}, v \in S_{x}$, projects isomorphically onto the tangent space at $[v]$ of

$$
\mathbb{P}_{x}=T_{x} M / \mathbb{C}^{*}=S_{x} / T^{1} .
$$

Hence the Levi form $\mathcal{L}_{x}$ induces an Hermitian metric $\hat{\mathcal{L}}_{x}$ on each tangent projective space $\mathbb{P}_{x}$. It is not difficult to see that $\hat{\mathcal{L}}_{x}$ is indeed a Kähler metric and that it depends smoothly on the point $x$ of $M$.

3.2. Adapted unitary frames of an $\mathbf{H}$-sphere bundle. In the next definition we introduce the concept of adapted unitary frames of an $\mathrm{H}$-sphere bundle. In all formulas, for any frame $u=\left\{f_{0}, \ldots, f_{2 n-1}\right\}$ we use the symbols $f_{1}, \ldots, f_{2 n-1}$ also to denote the vectors in $T_{f_{0}}\left(T_{x} M\right)$ which correspond to the vectors of $u$ via the natural identification map $\imath_{f_{0}}: T_{x} M \rightarrow T_{f_{0}}\left(T_{x} M\right)$.

DEFinition 3.4. We say that a complex linear frame $u=\left\{f_{0}, \ldots, f_{2 n-1}\right\}$ at $x=\pi(u)$ is adapted to the $H$-sphere bundle $(S M, \rho)$ if

a) $f_{0} \in S_{x}$ and $f_{1}=J f_{0}$;

b) the vectors $f_{2}, \ldots, f_{2 n-1}$ span the maximal $J$-invariant subspace $\mathcal{D}_{f_{0}}$ of $T_{f_{0}} S_{x}$ 
c) the holomorphic vectors $e_{1}, \ldots, e_{n-1}$ constitute a unitary basis for $\mathcal{D}_{f_{0}}$ with respect to the Levi form $\mathcal{L}_{x}$ defined by $(3.1)$.

The subbundle $U_{\rho}(S M) \subset L^{\mathbb{C}}(M)$ of all adapted unitary frames of $(S M, \rho)$ is called the unitary frame bundle of $(S M, \rho)$.

If $(S M, \rho)$ is the $\mathrm{H}$-sphere bundle of the complex Finsler manifold $(M, J, F)$, its unitary frame bundle is denoted by $U_{F}(M)$.

It is immediate that $\pi: U_{\rho}(S M) \rightarrow M$ is a subbundle of $L^{\mathbb{C}}(M)$ on which $U_{n-1}$ acts freely and fiber preserving.

Moreover, if $(S M, \rho)$ is circular, then for any fiber $\mathbb{V}_{x}=\pi^{-1}(x) \subset U_{\rho}(S M)$, the quotient $\mathbb{V}_{x} / T^{1}$ is equivalent to the unitary frame bundle $U_{\hat{\mathcal{L}}_{x}}\left(\mathbb{P}_{x}\right)$, where $\hat{\mathcal{L}}_{x}$ is the Kähler metric defined in Remark 3.3. This implies $U_{\rho}(S M)$ is a principal bundle only if all compact Kähler manifolds $\left(\mathbb{P}_{x}, \hat{\mathcal{L}}_{x}\right)$ are homogeneous spaces of a complex subgroup $G \subset G L_{n}(\mathbb{C})$ which properly contains $U_{n-1} \times T^{1}$. Since this condition is very strong, it is natural to expect that generically $U_{\rho}(S M)$ is not a principal subbundle of $L^{\mathbb{C}}(M)$.

In fact:

Proposition 3.5. The unitary frame bundle $U_{\rho}(S M) \subset L^{\mathbb{C}}(M)$ of an $H$-sphere bundle $(S M, \rho)$ is a principal subbundle if and only if it is the unitary frame bundle of an Hermitian metric $g$ on $(M, J)$.

Proof. If $U_{\rho}(S M)$ is a principal subbundle of $L^{\mathbb{C}}(M)$ with structure group $G \subset$ $G L_{n}(\mathbb{C})$, then the group $G$ verifies the following conditions:

i) it is compact;

ii) it acts transitively on each sphere $S_{x}=\mathbb{V}_{x} / U_{n-1}$;

iii) the isotropy subgroup of the $G$-action on each sphere $S_{x}=\mathbb{V}_{x} / U_{n-1}$ is $U_{n-1}$. From the list of the compact Lie groups acting transitively on a sphere ([MS], [Bo1], [Bo2]), it follows that the only group which verifies i), ii) and iii) is $G=U_{n}$. By standard arguments this implies that $U_{\rho}(S M)=U_{g}(M)$ for some Hermitian metric.

The following Lemma gives an alternative way to define the adapted unitary frames of a complex Finsler manifold.

Lemma 3.6. A frame $u=\left\{f_{i}\right\} \in L^{\mathbb{C}}(M)$ belongs to $U_{F}(M)$ if and only if the corresponding holomorphic frame $u^{10}=\left\{e_{\alpha}\right\}$ verifies

$$
h_{f_{0}}\left(e_{\alpha}, e_{\bar{\beta}}\right)=\delta_{\alpha \beta}
$$

for any $0 \leq \alpha, \beta \leq n-1$.

Proof. By definition of adapted frame, $u \in U_{F}(M)$ if and only if it verifies the follow three conditions for $1 \leq \lambda, \mu \leq n-1$ :
a) $F^{2}\left(f_{0}\right)=1$;
b) $\left.e_{\lambda}\left(F^{2}\right)\right|_{f_{0}}=0$;
c) $\left.\mathcal{L}_{x}\right|_{f_{0}}\left(e_{\lambda}, e_{\mu}\right)=\widetilde{\mathrm{h}}_{f_{0}}\left(e_{\lambda}, e_{\bar{\mu}}\right)=\delta_{\lambda \mu}$.

Since $\left.e_{0}\left(F^{2}\right)\right|_{f_{0}}=\left.D^{10}\left(F^{2}\right)\right|_{f_{0}}$, by Lemma $2.4 \mathrm{~d}$ ) and the identity between $\mathrm{h}$ and $\tilde{\mathrm{h}}$ on the tangent spaces of the Finsler spheres, the conditions a), b) and c) can be rewritten in the form

$$
\left.\left.\left.a^{\prime}\right) \mathrm{h}_{f_{0}}\left(e_{0}, e_{\overline{0}}\right)=1 ; \quad b^{\prime}\right) \mathrm{h}_{f_{0}}\left(e_{\lambda}, e_{\overline{0}}\right)=0 ; \quad c^{\prime}\right) \mathrm{h}_{f_{0}}\left(e_{\lambda}, e_{\bar{\mu}}\right)=\delta_{\lambda \mu},
$$

which is simply (3.2). 
From Lemma 3.6 and Proposition 3.5, it follows that $U_{F}(M)$ is a principal bundle if and only if $F$ is associated with an Hermitian metric $g$ and that in this case $U_{F}(M)=$ $U_{g}(M)$.

\subsection{Linear and non-linear connections of Hermitian type.}

Definition 3.7. Let $P \subset L^{\mathbb{C}}(M)$ be a subbundle of $L^{\mathbb{C}}(M), \imath: P \rightarrow L^{\mathbb{C}}(M)$ be the immersion map and $\hat{J}$ the standard complex structure of $L^{\mathbb{C}}(M)$. Then:

(1) a (non-linear) connection on $P \subseteq L^{\mathbb{C}}(M)$ is a distribution $\mathcal{H}$, of real dimension $2 n=\operatorname{dim} M$, complementary to the vertical distribution;

(2) if $P$ is a principal $G$-bundle, the connection $\mathcal{H}$ is called linear if it is $G$ invariant; if $P$ is the unitary frame bundle $U_{\rho}(S M)$ of a circular $\mathrm{H}$-sphere bundle $(S M, \rho), \mathcal{H}$ is called nice if it is $U_{n-1} \times T^{1}$-invariant;

(3) a nice (non-linear) connection $\mathcal{H}$ on $U_{\rho}(S M)$ is called isometrically invariant if for any biholomorphic isometry $f$ of $(S M, \rho)$, the lift $\hat{f}$ on $L^{\mathbb{C}}(M)$ leaves $\mathcal{H}$ invariant;

(4) a (non-linear) connection $\mathcal{H}$ is called of Hermitian type if it is $\hat{J}$-invariant, i.e. for any $u \in P$

$$
\hat{J}\left(\imath_{*}\left(\mathcal{H}_{u}\right)\right)=\imath_{*}\left(\mathcal{H}_{u}\right)
$$

(5) the connection form of a (non-linear) connection $\mathcal{H}$ is the unique $\mathfrak{g l}_{\mathfrak{n}}(\mathbb{C})$ valued 1 -form $\omega$ on $P$, which vanishes on $\mathcal{H}$ and verifies

$$
\omega\left(A_{u}^{*}\right)=A
$$

for any $u \in P$ and any $A$ in the algebraic vertical subspace $\mathfrak{g}_{u} \subset \mathfrak{g l}_{\mathfrak{n}}(\mathbb{C})$.

Remark 3.8. If $\mathcal{H}$ is a nice (non-linear) connection on $U_{\rho}(S M)$, for any curve

$$
\gamma:[a, b] \rightarrow \mathbb{P} T M=U_{\rho}(S M) / U_{n-1} \times T^{1},
$$

which projects on a given curve $\gamma_{o}$ in $M$, and any frame $u \in \pi^{-1}\left(\gamma_{a}\right) \subset U_{\rho}(S M)$, there exists a unique horizontal curve $\hat{\gamma}:[a, b] \rightarrow U_{\rho}(S M)$, which is tangent to $\mathcal{H}$, projecting onto $\gamma$ and with $\hat{\gamma}_{a}=u$. Since $\mathcal{H}$ is $U_{n-1} \times T^{1}$-invariant, if $\hat{\gamma}_{a}^{10}=u^{10}=\left\{e_{0}, \ldots, e_{n-1}\right\}$ and $\hat{\gamma}_{b}^{10}=\left\{e_{0}^{\prime}, \ldots, e_{n-1}^{\prime}\right\}$, the linear map

$$
T_{\gamma}: T_{x} M \rightarrow T_{x^{\prime}} M, \quad X=X^{i} e_{i} \stackrel{T_{\gamma}}{\mapsto} X^{i} e_{i}^{\prime}
$$

does not depend on the frame $u$, but only on the curve $\gamma$. We call it the parallel transport along $\gamma$.

Furthermore, if $U_{\rho}(S M)$ is a principal subbundle of $L^{\mathbb{C}}(M)$ and $\mathcal{H}$ is a linear connection, the parallel transport (3.5) depends just on the curve $\gamma_{o}:[a, b] \rightarrow M$ which is obtained by projecting on $M$ the curve $\gamma$ of $\mathbb{P} T M$. In particular, (3.5) is the classical parallel transport associated to a linear connection and it defines a linear covariant derivation on $M$.

If $\mathcal{H}$ is non-linear, the parallel transport (3.5) defines the following non-linear covariant derivation $\nabla$ on $M$ : let $Y$ be a local vector field on $M, \hat{X}$ a vector in $T_{[v]} \mathbb{P} T M$ and $\gamma:[a, b] \rightarrow \mathbb{P} T M$ a curve such that $\dot{\gamma}_{0}=\hat{X} ;$ then

$$
\nabla_{\hat{X}} Y=\lim _{h \rightarrow 0} \frac{1}{h}\left[T_{\left.\gamma\right|_{[0, h]} ^{-1}}\left(Y_{x_{h}}\right)-Y_{x}\right]
$$

where $x$ and $x_{h}$ are the base points of $\gamma_{0}=[v]$ and $\gamma_{h}$, respectively. If we denote by

$$
\hat{\pi}: U_{\rho}(S M) \rightarrow \mathbb{P} T M=U_{\rho}(S M) / U_{n-1} \times T^{1}
$$


the standard projection map, then $\left.\nabla_{\hat{X}} Y\right|_{v}$ is equal to

$$
\left.\nabla_{\hat{X}} Y\right|_{v}=u\left(\mathcal{X}_{u} \theta(\mathcal{Y})+\omega_{u}(\mathcal{X}) \cdot \theta_{u}(\mathcal{Y})\right)
$$

where $\omega$ is the connection form of $\mathcal{H}, u$ is any frame in $\hat{\pi}^{-1}(v) \subset U_{\rho}(S M)$ and $\mathcal{X}$ and $\mathcal{Y}$ are two vector fields on $U_{\rho}(S M)$ such that $\hat{\pi}_{*}(\mathcal{X})_{v}=\hat{X}$ and $\pi_{*}(\mathcal{Y})=Y$.

The derivation (3.7) is a linear function of the vector $\hat{X}$, not of just its projection to $T M$ : this is in clear contrast with the properties of linear connections.

\subsection{The non-linear Hermitian connection of a complex Finsler mani- fold.}

Theorem 3.9. For any complex Finsler manifold $(M, J, F)$, the unitary frame bundle $U_{F}(M)$ admits a unique non-linear connection of Hermitian type.

This non-linear connection of Hermitian type is nice and isometrically invariant.

We call the connection of Theorem 3.9 the non-linear Hermitian connection of $(M, J, F)$.

Proof. ${ }^{1}$ First of all, notice that if $f: M \rightarrow M$ is a local biholomorphism and $\hat{f}: L(M) \rightarrow L(M)$ is the lifted map on the linear frame bundle, than clearly $\hat{f}$ maps $L^{\mathbb{C}}(M)$ into itself and it is a local biholomorphism w.r.t. to the standard complex structure $\hat{J}$. Therefore any local biholomorphic isometry $f$ of $(M, J, F)$ is so that $\hat{f}\left(U_{F}(M)\right) \subset U_{F}(M)$ and $\hat{f}_{*}$ transforms any $\hat{J}$-invariant non-linear connection into another $\hat{J}$-invariant non-linear connection. Hence, if there exists a unique non-linear connection of Hermitian type, this connection is isometrically invariant.

Consider now the distribution $\mathcal{H} \subset T U_{F}(M)$ of all maximal $\hat{J}$-invariant subspaces, i.e. such that for any $u \in U_{F}(M)$

$$
\mathcal{H}_{u}=T_{u} U_{F}(M) \cap \hat{J}\left(T_{u} U_{F}(M)\right) .
$$

We want to prove that such distribution constitutes a non-linear connection, i.e. it is transversal to the vertical distribution and with $\operatorname{dim} \mathcal{H}_{u}=2 n$ at any frame $u$. Since any $\hat{J}$-invariant distribution $\mathcal{H}^{\prime}$ is included in $\mathcal{H}$, it follows immediately that $\mathcal{H}$ is a unique such non-linear connection. Furthermore, since $U_{n-1} \times T^{1} \subset G L_{n}(\mathbb{C})$ acts holomorphically on $L^{\mathbb{C}}(M)$, it follows also that $\mathcal{H}$ is $U_{n-1} \times T^{1}$-invariant and therefore is a nice non-linear connection.

To prove the claim we need the following Lemma.

Lemma 3.10. Let $x \in M$ and let $\mathbb{L}_{x}=\pi^{-1}(x) \subset L^{\mathbb{C}}(M)$ and $\mathbb{V}_{x}=\pi^{-1}(x) \subset$ $U_{F}(M)$ be the fibers of $L^{\mathbb{C}}(M)$ and $U_{F}(M)$ over $x$, respectively.

Then $\mathbb{V}_{x}$ is a maximally totally real submanifold of $\mathbb{L}_{x}$, i.e. for any $u \in \mathbb{V}_{x}$,

$$
T_{u} \mathbb{V}_{x} \cap \hat{J}\left(T_{u} \mathbb{V}_{x}\right)=\{0\} \quad \text { and } \quad T_{u} \mathbb{V}_{x} \cup \hat{J}\left(T_{u} \mathbb{V}_{x}\right)=T_{u} \mathbb{L}_{x}
$$

Proof. Let us identify the space of holomorphic vectors $T_{x}^{10} M \subset T_{x}^{\mathbb{C}} M$ with $\mathbb{C}^{n}$ and let $G r_{n-1}\left(\mathbb{C}^{n}\right)$ be the Grassmanian of $(n-1)$-dimensional complex subspaces in $T_{x}^{10} M=\mathbb{C}^{n}$. Let also denote by $J_{o}$ the standard complex structure of $\mathbb{C}^{n} \times G r_{n-1}\left(\mathbb{C}^{n}\right)$.

Consider now the holomorphic surjective map

$$
\sigma: \mathbb{L}_{x} \rightarrow \mathbb{C}^{n} \times G r_{n-1}\left(\mathbb{C}^{n}\right), \quad \sigma(u)=\left(e_{0}, \operatorname{span}\left\{e_{1}, \ldots, e_{n-1}\right\}\right)
$$

\footnotetext{
${ }^{1}$ For the following short and elegant way for proving Theorem 3.1, we are indebted to a kind suggestion of an anonymous referee
} 
where we denote by $u^{10}=\left\{e_{0}, \ldots, e_{n-1}\right\}$ the holomorphic basis determined by the frame $u$. It is clear that $\sigma: \mathbb{L}_{x} \rightarrow \mathbb{C}^{n} \times G r_{n-1}\left(\mathbb{C}^{n}\right)$ is a principle bundle with structure group $G L_{n}(\mathbb{C})$ and base $\mathbb{C}^{n} \times G r_{n-1}\left(\mathbb{C}^{n}\right)$.

Let $S_{x} \subset T_{x} M$ be the Finsler sphere at $x$ and let $Q \subset \mathbb{C}^{n} \times G r_{n-1}\left(\mathbb{C}^{n}\right)$ be the submanifold

$$
Q=\left\{\left(v^{10}, p\right) \in \mathbb{C}^{n} \times G r_{n-1}\left(\mathbb{C}^{n}\right): v \in S_{x}, p \subset T_{x}^{10} M \cap T_{v}^{\mathbb{C}} S_{x}\right\} .
$$

By definition, $\sigma\left(\mathbb{V}_{x}\right)=Q$ and the projection

$$
\left.\sigma\right|_{\mathbb{V}_{x}}: \mathbb{V}_{x} \rightarrow Q
$$

makes $\mathbb{V}_{x}$ a principle bundle over $Q$ with structure group $U_{n-1}$.

Since $S_{x}$ is strongly pseudo-convex, by a result of Webster (see [We1]), $Q$ is a maximally totally real submanifold of $\mathbb{C}^{n} \times G r_{n-1}\left(\mathbb{C}^{n}\right)$. Since also $U_{n-1}$ is a maximally totally real submanifold of $G L_{n}(\mathbb{C})$ and $\sigma$ is holomorphic, it follows that $\mathbb{V}_{x}$ is a maximally totally real submanifold as well.

Let us now prove the claim. From Lemma 3.10, it follows immediately that $\mathcal{H}$ is transversal to the vertical distribution, that is for any $u \in U_{F}(M)$

$$
\mathcal{H}_{u} \cap T_{u} \mathbb{V}_{\pi(x)}=\{0\} .
$$

Furthermore, $\operatorname{dim} \mathbb{L}_{x}=2 \operatorname{dim} \mathbb{V}_{x}$ and hence $\operatorname{dim} L^{\mathbb{C}}(M)=\operatorname{dim} M+\operatorname{dim} \mathbb{L}_{x}=2 n+$ $2 \operatorname{dim} \mathbb{V}_{x}$. Since $\operatorname{dim} U_{F}(M)=\operatorname{dim} M+\operatorname{dim} \mathbb{V}_{x}=2 n+\operatorname{dim} \mathbb{V}_{x}$ it follows that at any $u \in U_{F}(M)$

$$
2 n+2 \operatorname{dim} \mathbb{V}_{\pi(u)} \geq T_{u} U_{F}(M)+\hat{J}\left(T_{u} U_{F}(M)\right)-\operatorname{dim} H_{u}=4 n+2 \operatorname{dim} \mathbb{V}_{x}-\operatorname{dim} \mathcal{H}_{u}
$$

that is $\operatorname{dim} \mathcal{H}_{u} \geq 2 n$. Since $\mathcal{H}_{u}$ is transversal with $T_{u} \mathbb{V}_{\pi(v)}$, we conclude that $\operatorname{dim} \mathcal{H}_{u}=$ $2 n$ for any $u$ and hence that $\mathcal{H}$ is a non-linear connection.

Since the unitary frame bundle $U_{g}(M)$ of an Hermitian metric $g$ on $(M, J)$ coincides with the unitary frame bundle $U_{F}(M)$ of the Finsler metric $F(v)=\sqrt{g(v, v)}$, Theorem 3.9 gives the following classical result as an immediately corollary (see e.g. [KN], vol. II): an Hermitian manifold $(M, J, g)$ has a unique Hermitian linear connection.

\section{The absolute parallelism on $U_{F}(M)$ and Kobayashi's theorem.}

4.1. Pseudo-hermitian structures on a real hypersurface. In this subsection, we recall the definition of pseudo-hermitian structure on CR manifold of codimension one and Webster's theorem on the existence and uniqueness of an invariant linear connection for any pseudo-hermitian structures. This result is essential for the construction of an invariant absolute parallelism on the unitary frame bundle $U_{F}(M)$ of a complex Finsler manifold.

Let $S$ be a $(2 n-1)$-dimensional manifold. $A$ CR structure on $S$ is a pair $(\mathcal{D}, J)$, where $\mathcal{D} \subset T S$ is a distribution and $J$ is a smooth family of complex structures $J_{p}$ on the subspaces $\mathcal{D}_{p} \subset T_{p} M$. It is called integrable if the holomorphic distribution $\mathcal{D}^{10} \subset \mathcal{D}^{\mathbb{C}}$ defined by $J$ is closed under Lie brackets. It is called of codimension $p$ if the distribution $\mathcal{D}$ is of codimension $p$.

An CR structure $(\mathcal{D}, J)$ of codimension one is called Levi non-degenerate if $\mathcal{D}$ is a contact distribution, i.e. if for any local 1 -form $\theta$ such that $\operatorname{ker} \theta=\mathcal{D}$, then $d \theta_{p}$ is non degenerate on $\mathcal{D}_{p}$ at any point $p$ where $\theta$ is defined. 
DEFINITION 4.1. [We] A pseudo-hermitian structure on $S$ is a pair $((S, \mathcal{D}, J) ; \theta)$ where $(S, \mathcal{D}, J)$ is a codimension one Levi non-degenerate $\mathrm{CR}$ structure and $\theta$ is a 1 -form on $S$ such that $\operatorname{ker} \theta_{p}=\mathcal{D}_{p}$ for any $p \in S$.

A pseudo-hermitian transformation of $((S, \mathcal{D}, J) ; \theta)$ is a diffeomorphism $f: S \rightarrow S$ such that $f_{*}(\mathcal{D}) \subset \mathcal{D},\left.f_{*} \circ J\right|_{\mathcal{D}}=\left.J \circ f_{*}\right|_{\mathcal{D}}$ and $f^{*} \theta=\theta$.

In the following, we denote a pseudo-hermitian structure only by a pair $(S, \theta)$.

A standard example of pseudo-hermitian structure is the following. Let $S$ be a smooth real hypersurface in $\mathbb{C}^{n}$ and $(\mathcal{D}, J)$ the codimension one CR structure determined by the maximal $J_{o}$-invariant subspaces in $T S$ and the complex structures determined by the complex structure $J_{o}$ of $\mathbb{C}^{n}$. Assume also that $\rho$ is a smooth defining function for $S$, i.e. $S=\left\{p \in \mathbb{C}^{n}: \rho(p)=0\right\}$. The 1-form $\theta^{\rho}$

$$
\theta_{p}^{\rho}(v)=d \rho_{p}\left(J_{o} v\right)
$$

vanishes exactly on the vectors on $\mathcal{D}$. Hence if $(S, \mathcal{D}, J)$ is Levi non-degenerate, then $\left(S, \theta^{\rho}\right)$ is a pseudo-hermitian structure.

DeFinition 4.2. Let $(S, \theta)$ be a pseudo-hermitian structure and let $u=\left\{f_{1}\right.$, $\left.\ldots, f_{2 n-1}\right\}$ a linear frame at a point $p \in S$. The frame $u$ is called adapted to $(S, \theta)$ if

a) $\theta\left(f_{1}\right)=1$ and $\theta\left(f_{i}\right)=0$ for $2 \leq i \leq 2 n-1$;

b) $d \theta\left(f_{i}, f_{j}\right)=\delta_{i j}$ for $2 \leq i, j \leq 2 n-1$;

c) $J f_{2 i}=f_{2 i+1}$ for $1 \leq i \leq n-1$.

The collection $U_{\theta}(S)$ of all adapted frames of frames of a pseudo-hermitian structure $(S, \theta)$ is called unitary frame bundle of $(S, \theta)$.

Conditions b) and c) can be restated claiming that the vectors $e_{\alpha}=f_{2 \alpha}-i f_{2 \alpha+1}$, with $\alpha=1, \ldots, n-1$, constitute a holomorphic basis for $\mathcal{D}_{p}^{10} \subset \mathcal{D}_{p}^{\mathbb{C}}$, which is unitary w.r.t. the Levi form $\mathcal{L}(X, Y)=d \theta_{p}(X, \bar{Y})$. It can be checked that $U_{\theta}(S)$ is a principal subbundle of $L(S)$ with structure group $U_{q, q^{\prime}}$, where $\left(q, q^{\prime}\right)$ is the signature of the Levi form $\mathcal{L}$ (see also [We]).

For any linear connection $\mathcal{H}$ on $U_{\theta}(S)$ and any frame $u=\left\{f_{1}, \ldots, f_{2 n-1}\right\} \in U_{\theta}(S)$, let us denote by $p_{u}=\left.\pi_{*}\right|_{\mathcal{H}_{u}}: \mathcal{H}_{u} \rightarrow T_{\pi(u)} S$ the restriction of $\pi_{*}$ on $\mathcal{H}$. We also denote by $\left\{\tilde{f}_{i}=p_{u}^{-1}\left(f_{i}\right)\right\}$ the basis of $\mathcal{H}_{u}$ which projects onto the vectors of $u$.

Since $p_{u}$ is a linear isomorphism between $\mathcal{H}_{u}$ and $T_{\pi(u)} S$, we may always consider the subspace $\tilde{\mathcal{D}}_{u}=p_{u}^{-1}\left(\mathcal{D}_{\pi(u)}\right) \subset \mathcal{H}_{u}$ and the complex structure $\tilde{J}$ on $\tilde{\mathcal{D}}_{u}$, defined by

$$
\tilde{J} \cdot v=\left(p_{u}^{-1} \circ J \circ p_{u}\right)(v) .
$$

$(\tilde{\mathcal{D}}, \tilde{J})$ are called the horizontal lifts of the $C R$ structure $(\mathcal{D}, J)$.

Definition 4.3. Let $\mathcal{H}$ be a linear connection on $U_{\theta}(S)$ and let $(\tilde{\mathcal{D}}, \tilde{J})$ the corresponding horizontal lift of the CR structure of $S$. Let also $\tilde{e}_{\alpha}$ the holomorphic vector fields in $\tilde{\mathcal{D}}^{10}$ defined by

$$
\left.\tilde{e}_{\alpha}\right|_{u}=\left.\tilde{f}_{2 \alpha}\right|_{u}-\left.i \tilde{f}_{2 \alpha+1}\right|_{u} \in \tilde{\mathcal{D}}_{u}^{10}, \quad 1 \leq \alpha \leq n-1 .
$$

We say that $\mathcal{H}$ is of Webster type if:

a) any Lie bracket between two vector fields of the holomorphic distribution $\tilde{\mathcal{D}}^{10} \subset \tilde{\mathcal{D}}^{\mathbb{C}}$ is 0 ;

b) $\pi_{*}\left(\left[\tilde{e}_{\alpha}, \overline{\tilde{e}_{\beta}}\right]\right)=-\sqrt{-1} \delta_{\alpha \beta} \pi_{*}\left(\tilde{f}_{1}\right)$ for any $1 \leq \alpha, \beta \leq n-1$;

c) for any $1 \leq \alpha \leq n-1$ the complex vector field $T_{\alpha}=\left[\tilde{e}_{\alpha}, \tilde{f}_{1}\right]$ is so that $\pi_{*}\left(T_{\alpha}\right)$ takes values in $\mathcal{D}^{01}=\overline{\mathcal{D}^{10}}$ at all points. 
We call the vector field $T_{\alpha}=\left[\tilde{e}_{\alpha}, \tilde{f}_{1}\right]$ the $\alpha$-th component of the torsion of $\mathcal{H}$.

We conclude with the following important result by S. Webster.

THEOREM 4.4. [We] If $(S, \theta)$ is a pseudo-hermitian structure, there exists exactly one linear connection of Webster type on $U_{\theta}(S)$. This connection is invariant under the group $\operatorname{Aut}(S, \theta)$ of pseudo-hermitian transformations of $(S, \theta)$.

Moreover, the $\alpha$-th components of the torsion $T_{\alpha}=\left[\tilde{e}_{\alpha}, \tilde{f}_{1}\right]$ of the connection of Webster type vanish identically if and only if the vector field $f_{1}$, given by the first vectors of all adapted frames, is an infinitesimal $C R$ transformations of $S$.

We call such unique linear connection the Webster connection of $(S, \theta)$.

4.2. The generalized fundamental vector fields on the unitary frame bundle of an $\mathrm{H}$-sphere bundle. Consider a circular $\mathrm{H}$-sphere bundle $(S M, \rho)$. For any $x \in M$, let $\rho_{x}$ be the restriction $\rho_{x}=\left.\rho\right|_{T_{x}} M$ and $\theta_{x}=\theta_{x}^{\rho}$ the 1-form on $S_{x}$ defined by (4.1). Each pair $\left(S_{x}, \theta_{x}\right)$ is a pseudo-hermitian structure.

If $u=\left\{f_{0}, f_{1}, \ldots, f_{2 n}\right\} \subset T_{x} M$ is an adapted unitary frame of $U_{\rho}(S M)$ and if we identify the vectors $\left\{f_{1}, \ldots, f_{2 n}\right\}$ with the corresponding vectors at $T_{f_{0}} S_{x}$, we immediately see that $u$ is also an adapted frame for the pseudo-hermitian structure $\left(S_{x}, \theta_{x}\right)$. In other words, the fiber $\mathbb{V}_{x}=\pi^{-1}(x) \in U_{\rho}(S M)$ can be identified with the unitary frame bundle $U_{\theta_{x}}\left(S_{x}\right)$.

Let $\mathcal{W}_{x}$ be the Webster connection on $\mathbb{V}_{x} \simeq U_{\theta_{x}}\left(S_{x}\right)$. Notice that the flow of the vector field $f_{1}$ on $S_{x}$ coincides with 1-parameter group of transformations given by $T^{1}$. Therefore by Theorem 4.4 each component of the torsion of the Webster connection $\mathcal{W}_{x}$ vanishes identically.

Using the Webster connection $\mathcal{W}_{x}$ and the fundamental vector fields associated to the Lie algebra $\mathfrak{u}_{n-1} \oplus \mathbb{R}=\operatorname{Lie}\left(U_{n-1} \times T^{1}\right)$ we define an absolute parallelism on any fiber $\mathbb{V}_{x} \subset U_{\rho}(S M)$ as follows.

DEFINITION 4.5. Let $U_{\rho}(S M)$ be the unitary frame bundle of a circular H-sphere bundle $(S M, \rho)$. For any $x \in M$, let also $\mathcal{W}_{x}$ be the Webster connection on the fiber $\mathbb{V}_{x}=\pi^{-1}(x) \simeq U_{\theta_{x}}\left(S_{x}\right)=U_{\theta}\left(S_{x}\right)$ and $\hat{\pi}: \mathbb{V}_{x} \rightarrow S_{x}=\mathbb{V}_{x} / U_{n-1}$ the standard projection map.

For any element $X \in W \oplus\left(\mathfrak{u}_{n-1} \oplus \mathbb{R}\right)$ we associate a vertical vector field $\tilde{X}$ of $U_{\rho}(S M)$ associated as follows:

(1) if $X \in \mathfrak{u}_{n-1} \oplus \mathbb{R}$, we set $\tilde{X}=X^{*}$;

(2) if $X \in W\left(=\mathbb{C}^{n-1}\right)$, we set $\tilde{X}$ to be the vector field so that, for any frame $u$, $\tilde{X}_{u}$ is the unique vector in $\left(\mathcal{W}_{\pi(u)}\right)_{u}$, such that $\hat{\pi}_{*}\left(\tilde{X}_{u}\right)=u(X) \in T_{f_{0}} S_{x}$;

(3) for any two vectors $X, X^{\prime} \in W \oplus\left(\mathfrak{u}_{n-1} \oplus \mathbb{R}\right)$, we set $\widetilde{X+X^{\prime}}=\tilde{X}+\tilde{X}^{\prime}$. Any vertical vector field $\tilde{X}$ is called generalized fundamental vector field.

If $\left\{\epsilon_{i}, E_{j}\right\}$ is a basis for $W \oplus\left(\mathfrak{u}_{n-1} \oplus \mathbb{R}\right)$, the generalized fundamental vector fields $\left\{\tilde{\epsilon}_{i}, \tilde{E}_{i}\right\}$ are linearly independent at all points and they give an absolute parallelism on each fiber $\mathbb{V}_{x}$. By construction, the vector fields $\left\{\tilde{\epsilon}_{i}, \tilde{E}_{i}\right\}$ are mapped into themselves by any biholomorphic isometry of $(S M, \rho)$.

One may also check that if $U_{\rho}(S M)$ is a principal subbundle (and hence with structure group $U_{n}$; see Proposition 3.5), the generalized fundamental vector fields coincide with the fundamental vector fields of the elements in $\mathfrak{u}_{n}$.

4.3. The absolute parallelism on $U_{F}(M)$ and the isometry group of 
$(M, J, F)$. Let $U_{F}(M)$ be the unitary frame bundle of a complex Finsler manifold $(M, J, F)$ and $\mathcal{H}$ the non-linear Hermitian connection on $U_{F}(M)$.

At any frame $u=\left\{f_{0}, \ldots, f_{2 n-1}\right\} \in U_{F}(M)$ we denote by $\left.\hat{f}_{i}\right|_{u}$ the unique vectors in $\mathcal{H}_{u}$ which project onto $f_{i} \in T_{\pi(u)} M$. Let also $\left\{\epsilon_{i}\right\}$ the standard basis of $W=\mathbb{C}^{n-1}$, $t$ be a generator of $\mathbb{R}=\operatorname{Lie}\left(T^{1}\right)$ and $\left\{E_{j}\right\}$ a basis for $\mathfrak{u}_{n-1}$. Let also $\tilde{\epsilon}_{i}, \tilde{t}$ and $\tilde{E}_{j}$ the corresponding generalized fundamental vector fields on $U_{F}(M)$.

Then the set of vector fields

$$
\sigma^{\mathcal{H}}=\left\{\hat{f}_{i}, \tilde{\epsilon}_{j}, \tilde{t}, \tilde{E}_{k}\right\}
$$

is an absolute parallelism on $U_{F}(M)$. It is unique, up to a change of the generator $t$ and of the basis $\left\{E_{j}\right\}$ for $\mathfrak{u}_{n-1}$ and it is invariant under all local biholomorphic isometries of $(M, J, F)$.

We call $\sigma^{\mathcal{H}}$ the absolute parallelism associated to the Hermitian connection $\mathcal{H}$.

As a consequence of Kobayashi's theorem on the automorphism group of an absolute parallelism ([Ko]), the following result is easily obtained.

Proposition 4.6. Let $(M, J, F)$ be a complex Finsler manifold of complex dimension $n$ and let $\mathcal{H}$ the non-linear Hermitian connection of $(M, J, F)$.

(1) The local holomorphic isometries of $(M, J, F)$ are in 1-1 correspondence with the local diffeomorphisms of $U_{F}(M)$, defined on open saturated subsets $\pi^{-1}(\mathcal{U})$, which preserve the absolute parallelism $\sigma^{\mathcal{H}}$. For any local biholomorphic isometry $f$ the corresponding local diffeomorphism is the restriction on $U_{F}(M) \subset L^{\mathbb{C}}(M)$ of the diffeomorphism

$$
\hat{f}: L(M) \rightarrow L(M), \quad \hat{f}(u)=f_{*} \circ u .
$$

(2) The group $\operatorname{Iso}_{F}(M)$ of all biholomorphic isometries of $(M, J, F)$ is a Lie group of dimension less or equal to

$$
\operatorname{dim}_{\mathbb{R}} U_{F}(M)=\operatorname{dim}_{\mathbb{R}} V+\operatorname{dim}_{\mathbb{R}} W+\operatorname{dim}_{\mathbb{R}}\left(\mathfrak{u}_{n-1} \oplus \mathbb{R}\right)=n^{2}+2 n .
$$

(3) $\operatorname{dim}_{\mathbb{R}} I \operatorname{sos}_{F}(M)=n^{2}+2 n$ if and only if $F$ is associated with a Kähler metric $g$ and $(M, J, g)$ is $\left(\mathbb{C} P^{n}, g_{c^{2}}\right),\left(\mathbb{C}^{n}, g_{0}\right)$ or $\left(B^{n}, g_{-c^{2}}\right)$, where $g_{c^{2}}, g_{0}$ and $g_{-c^{2}}$ denote the Kähler metrics with constant holomorphic sectional curvature $c^{2}, 0$ and $-c^{2}$, respectively.

Proof. (1) It is proved with the same arguments of Proposition 3.3 in [Sp]; they are very similar to the arguments of the proof of Proposition VI. 3.1 in [KN] Vol. I.

(2) It is an immediate corollary of (1) and of Theorem I.3.2 in [Ko1].

(3) If $\operatorname{dim}_{\mathbb{R}} I s o_{F}(M)=n^{2}+2 n$, then $U_{F}(M)$ is a principal subbundle of $L^{\mathbb{C}}(M)$ and any isotropy subgroup $I s_{F}(M)_{x}$ acts transitively on any fiber $\mathbb{V}_{x}$ of $U_{F}(M)$. By Proposition 3.5 and Lemma 3.6 it follows that $I s o_{F}(M)_{x} \simeq U_{n}$ for any $x \in M$ and that $F$ is associated with an Hermitian metric $g$. Since for any $x \in M$ we have that $I_{s o_{F}}(M)_{x}=I s o_{g}(M)_{x} \simeq U_{n}$, we also have that $(M, J, g)$ is an Hermitian symmetric space, $g$ is Kähler and the holomorphic sectional curvature is constant.

We claim now that $M$ is simply connected. Suppose not and let $\pi: \tilde{M} \rightarrow M$ be its universal covering map and $\tilde{g}=\pi^{*} g$. For any $x \in M$ and any $y \in \pi^{-1}(x)$, the isotropy subgroup $I s o_{g}(M)_{x} \simeq U_{n}$ is embedded into $I s o_{\tilde{g}}(\tilde{M})_{y}$ and hence $I s o_{\tilde{g}}(M)_{y}=$ $I_{s o_{g}}(M)_{x} \simeq U_{n}$. This implies that any deck transformation $\Gamma$ belongs to the normalizer $N_{I s o_{\tilde{g}}(\tilde{M})}\left(\operatorname{Iso}_{\tilde{g}}(\tilde{M})_{y}\right)$ of the subgroup $I s o_{\tilde{g}}(M)_{y}$ in $I s o_{\tilde{g}}(\tilde{M})$. Suppose now that $\tilde{g}$ has positive holomorphic sectional curvature. By the classification of simply connected complex space forms, $\tilde{M}$ is $\mathbb{C} P^{n}, I s o_{\tilde{g}}(\tilde{M})=S U_{n+1}$ and $N_{I s o_{\tilde{g}}(\tilde{M})}\left(I s o_{\tilde{g}}(\tilde{M})_{y}\right)=$ 
$N_{S U_{n+1}}\left(U_{n}\right)=U_{n}$. This means that any deck transformation $\Gamma$ belongs to $I s o_{\tilde{g}}(M)_{y}$, and this cannot be because $\Gamma$ fixes no point. Suppose then that $\tilde{g}$ has non-positive holomorphic sectional curvature. In this case for any non-trivial deck transformation and any $h \in I \operatorname{sos}_{\tilde{g}}(M)_{y}$, there exists some $h^{\prime} \in I_{s o_{\tilde{g}}}(M)_{y}$ so that $h \circ \Gamma=\Gamma \circ h^{\prime}$ and hence $h(\Gamma(y))=\Gamma\left(h^{\prime}(y)\right)=\Gamma(y)$. Since $h$ fixes $y$ and $\Gamma(y)$, it fixes point by point the unique length minimizing geodesic between $y$ and $\Gamma(y)$. But this cannot be because $\operatorname{Iso}_{\tilde{g}}(\tilde{M})_{y}=U_{n}$ and it fixes no vector in $T_{y} \tilde{M}$.

Since $M$ is simply connected, the claim follows from the classification of simply connected complex space forms.

\section{The invariants of a complex Finsler manifolds.}

5.1. Notation. In all the following sections, the greek indices $\alpha, \beta, \gamma, \delta, \varepsilon$ always run between $0, \ldots, n-1$; the indices $\lambda, \mu, \nu, \rho, \sigma$ run between 1 and $n-1$.

We denote by $E_{\beta}^{\alpha}=\varepsilon_{\beta} \otimes \varepsilon^{\alpha}$ the elements of the standard basis of $\mathfrak{g l}_{\mathfrak{n}}(\mathbb{C})$. An element $A=A_{\beta}^{\alpha} E_{\alpha}^{\beta} \in \mathfrak{g l}_{\mathfrak{n}}(\mathbb{C})$ can be also expressed using just the complex matrix $A_{\beta}^{\alpha}$.

For any adapted unitary frame $u=\left\{f_{i}\right\}$ and corresponding holomorphic frame $u^{10}=\left\{e_{\alpha}\right\}$, we set

$$
h_{\alpha \beta}(u)=\mathrm{h}_{f_{0}}\left(e_{\alpha}, e_{\beta}\right), H_{\alpha \beta \gamma}(u)=\mathrm{H}_{f_{0}}\left(e_{\alpha}, e_{\beta}, e_{\gamma}\right), H_{\alpha \beta \gamma \delta}(u)=\mathbf{H}_{f_{0}}\left(e_{\alpha}, e_{\beta}, e_{\gamma}, e_{\delta}\right) .
$$

The symbols $h_{\bar{\alpha} \bar{\beta}}(u), H_{\alpha \beta \bar{\gamma}}(u), H_{\alpha \bar{\beta} \bar{\gamma}}(u)$, etc. have analogous meanings.

On $U_{F}(M)$ we have the following distributions and CR structures:

- $\mathcal{H}$ is the non-linear Hermitian connection;

- $\mathcal{W}=\bigcup_{x \in M} \mathcal{W}_{x}$ is the distribution obtained as union of the Webster connections $\mathcal{W}_{x}$ of the fibers $\mathbb{V}_{x}=\pi^{-1}(x)=U_{\theta_{x}}\left(S_{x}\right)$;

- $(\tilde{\mathcal{D}}, \tilde{J})$ is the CR structure given by $\tilde{\mathcal{D}}=\bigcup_{x \in M} \tilde{D}_{x}$ and $\tilde{J}=\bigcup_{x \in M} \tilde{J}_{x}$, where each $\left(\tilde{\mathcal{D}}_{x}, \tilde{J}_{x}\right)$ is the horizontal lift in $\mathcal{W}_{x}$ of the CR structure of the Finsler sphere $S_{x}$ (see Definition 4.3).

Notice that $(\mathcal{H}, \hat{J})$ and $(\tilde{\mathcal{D}}, \tilde{J})$ are both integrable CR structures on $U_{F}(M)$ (see $\S 4.1$ ).

$\omega$ is the connection form of $\mathcal{H}$ (see Def. 3.7) and we define $\omega_{\beta}^{\alpha}$ as the $\mathbb{C}$-valued 1-forms on $U_{F}(M)$ which verify

$$
\omega=\sum_{\alpha, \beta} E_{\beta}^{\alpha} \otimes \omega_{\alpha}^{\beta}
$$

We also set $\omega_{\bar{\beta}}^{\bar{\alpha}}=\overline{\omega_{\beta}^{\alpha}}$. We call $\omega_{\beta}^{\alpha}$ and $\omega_{\bar{\beta}}^{\bar{\alpha}}$ the holomorphic and anti-holomorphic components of the connection form $\omega$.

We denote by $\mathcal{E}_{\beta}^{\alpha}$ and by $\mathcal{E}_{\bar{\beta}}^{\bar{\alpha}}$ the complex vector fields on $L^{\mathbb{C}}(M)$ defined by

$$
\mathcal{E}_{\beta}^{\alpha}=\frac{1}{2}\left[\left(E_{\beta}^{\alpha}\right)^{*}-\sqrt{-1}\left(J_{o} E_{\beta}^{\alpha}\right)^{*}\right], \quad \mathcal{E}_{\bar{\beta}}^{\bar{\alpha}}=\overline{\mathcal{E}_{\beta}^{\alpha}} .
$$

Note that, if we extend $\mathbb{C}$-linearly the 1 -forms $\omega_{\beta}^{\alpha}$ and $\omega_{\bar{\beta}}^{\bar{\alpha}}$, we have that

$$
\omega_{\beta}^{\alpha}\left(\mathcal{E}_{\gamma}^{\varepsilon}\right)=\delta_{\gamma}^{\alpha} \delta_{\beta}^{\varepsilon}, \quad \omega_{\bar{\beta}}^{\bar{\alpha}}\left(\mathcal{E}_{\gamma}^{\delta}\right)=0 .
$$

Moreover, we recall that by the properties of fundamental vector fields (see [KN])

$$
\mathcal{L}_{\left(E_{\beta}^{\alpha}\right)^{*}} \theta=-\sum_{\gamma}\left[E_{\beta}^{\alpha}, \varepsilon_{\gamma}\right] \otimes \theta^{\gamma}-\sum_{\gamma}\left[E_{\beta}^{\alpha}, \varepsilon_{\bar{\gamma}}\right] \otimes \theta^{\bar{\gamma}}
$$




$$
\mathcal{L}_{\left(J_{o} E_{\beta}^{\alpha}\right)^{*}} \theta=-\sum_{\gamma} \sqrt{-1}\left[E_{\beta}^{\alpha}, \varepsilon_{\gamma}\right] \otimes \theta^{\gamma}+\sqrt{-1} \sum_{\gamma}\left[E_{\beta}^{\alpha}, \varepsilon_{\bar{\gamma}}\right] \otimes \theta^{\bar{\gamma}}
$$

This implies that

$$
\mathcal{L}_{\mathcal{E}_{\beta}^{\alpha}} \theta=\frac{1}{2}\left[\mathcal{L}_{\left(E_{\beta}^{\alpha}\right)^{*}} \theta-\sqrt{-1} \mathcal{L}_{\left(J_{o} E_{\beta}^{\alpha}\right)^{*}} \theta\right]=-\varepsilon_{\beta} \otimes \theta^{\alpha}, \quad \mathcal{L}_{\mathcal{E}_{\bar{\beta}}^{\bar{\alpha}}} \theta=\overline{\mathcal{L}_{\mathcal{E}_{\beta}^{\alpha}} \theta}=-\varepsilon_{\bar{\beta}} \otimes \theta^{\bar{\alpha}} .
$$

For any fiber $\mathbb{V}_{x}=\pi^{-1}(x) \subset \mathbb{V}_{x}$, we denote by

$$
\hat{\pi}_{x}: \mathbb{V}_{x}=U_{\theta_{x}}\left(S_{x}\right) \rightarrow S_{x}=\mathbb{V}_{x} / U_{n-1}
$$

the standard projection map of $\mathbb{V}_{x}=U_{\theta_{x}}\left(S_{x}\right)$ onto $S_{x}=\mathbb{V}_{x} / U_{n-1}$.

For any $i=0, \ldots, 2 n-1, \hat{f}_{i}$ is the vector field in $\mathcal{H}$ such that at all $u \in U_{F}(M)$

$$
\left.\pi_{*}\left(\hat{f}_{i}\right)\right|_{u}=f_{i}=u\left(\epsilon_{i}\right) \in T_{\pi(u)} M .
$$

For any $a=2, \ldots, 2 n-1, \tilde{\epsilon}_{a}$ is the generalized fundamental vector fields which corresponds to elements of the real basis $\left\{\epsilon_{2}, \ldots, \epsilon_{2 n-1}\right\}$ of $W=\mathbb{C}^{n-1}$ (see $\S 2.1$ ). By construction, any vector field $\tilde{\epsilon}_{a}$ is vertical, it takes values in $\tilde{\mathcal{D}} \subset \mathcal{W}$ and $\tilde{J} \tilde{\epsilon}_{2 i}=\tilde{\epsilon}_{2 i+1}$.

Finally, we denote by $\hat{e}_{\alpha}$ and $\tilde{e}_{\lambda}$ the holomorphic vector fields

$$
\hat{e}_{\alpha}=\frac{1}{2}\left(\hat{f}_{2 \alpha}-\sqrt{-1} \hat{f}_{2 \alpha+1}\right), \quad \tilde{e}_{\lambda}=\frac{1}{2}\left(\tilde{\epsilon}_{2 \lambda}-\sqrt{-1} \tilde{\epsilon}_{2 \lambda+1}\right) .
$$

They coincide with the complex vector fields in $\mathcal{H}^{\mathbb{C}}$ and $\tilde{\mathcal{D}}^{\mathbb{C}} \subset \mathcal{W}^{\mathbb{C}}$, which are mapped by $\pi_{*}$ and $\hat{\pi}_{x *}$ onto the holomorphic vectors of the corresponding adapted frames of $T_{x} M$ and of $T_{\hat{\pi}_{x}(u)} S$, respectively.

5.2. The algebraic vertical subspaces of $U_{F}(M)$ and the distribution $\mathcal{W}$. We want to determine the algebraic vertical subspaces of $\mathfrak{g}_{u}$ (see definition in $\S 2.1$ ). For this we give the following technical lemma, which follows directly from definitions and Lemma $2.4 \mathrm{e}$ ).

LEMMA 5.1. For any choice of the indices $A, B, C$, the functions $H_{A B C}$ are totally symmetric w.r.t. to $A, B$ and $C$. Furthermore, for any $u=\left\{f_{0}, \ldots, f_{2 n-1}\right\}$,

$$
H_{\alpha \bar{\beta} 0}(u)=H_{\bar{\alpha} \beta \overline{0}}(u)=0, \quad H_{\alpha \beta 0}(u)=-h_{\alpha \beta}(u), \quad H_{\alpha \beta \overline{0}}(u)=h_{\alpha \beta}(u) .
$$

Now recall that for any $A \in \mathfrak{g l}_{\mathfrak{n}}(\mathbb{C})$ and any $u \in L^{\mathbb{C}}(M)$, the corresponding fundamental vector field $A_{u}^{*}$ at $u$ is equal to the tangent vector at $t=0$ of the curve $a(t)=u \circ e^{t A}$. Therefore, by Lemma 3.6, the element $A$ belongs to the algebraic vertical subspace $\mathfrak{g}_{u}$ if and only if

$$
\left.\frac{d}{d t}\left[\mathrm{~h}_{e^{t A} \cdot f_{0}}\left(e^{t A} \cdot e_{\alpha}, e^{t A} \cdot e_{\bar{\beta}}\right)\right]\right|_{t=0}=0 .
$$

Representing $A$ with the associated matrix $A_{\beta}^{\alpha}$, condition (5.2) can be written as:

$$
A_{\alpha}^{\gamma} \delta_{\gamma \beta}+\delta_{\alpha \gamma} A_{\bar{\beta}}^{\bar{\gamma}}+A_{0}^{\gamma} H_{\alpha \bar{\beta} \gamma}(u)+A_{\overline{0}}^{\bar{\gamma}} H_{\alpha \bar{\beta} \bar{\gamma}}(u)=0 .
$$

Using Lemma 5.1, we immediately obtain the following. 
Proposition 5.2. For any $u \in U_{F}(M)$, the algebraic vertical subspace $\mathfrak{g}_{u} \subset$ $\mathrm{gl}_{n}(\mathbb{C})$ is defined by the following equations:

$$
\begin{gathered}
A_{0}^{0}+A_{\overline{0}}^{\overline{0}}=0, \quad A_{\lambda}^{0}+A_{\overline{0}}^{\bar{\lambda}}+A_{0}^{\nu} h_{\lambda \nu}(u)=0 \\
A_{\lambda}^{\mu}+A_{\bar{\mu}}^{\bar{\lambda}}+A_{0}^{\nu} H_{\lambda \bar{\mu} \nu}(u)+A_{\overline{0}}^{\bar{\nu}} H_{\lambda \bar{\mu} \bar{\nu}}(u)=0 .
\end{gathered}
$$

Equations (5.4) are called the defining equations of the algebraic vertical subspace $\mathfrak{g}_{u}$.

For any $u \in U_{F}(M)$, consider the following basis for $\mathfrak{g}_{u}(\lambda \geq \mu)$ :

$$
\begin{aligned}
t= & J_{o} \cdot E_{0}^{0}, \\
E_{\lambda, \mu}^{R}= & E_{\mu}^{\lambda}-E_{\lambda}^{\mu}, \quad E_{\lambda, \mu}^{I}=J_{o} \cdot\left(E_{\mu}^{\lambda}+E_{\lambda}^{\mu}\right), \\
E_{2 \lambda}(u)= & E_{\lambda}^{0}-\left[\operatorname{Re}\left\{h_{\lambda \mu}(u)\right\}+\delta_{\lambda \mu}\right] E_{0}^{\mu}-\operatorname{Im}\left\{h_{\lambda \mu}(u)\right\} J_{o} \cdot E_{0}^{\mu} \\
& -\operatorname{Re}\left\{H_{\bar{\nu} \mu \lambda}(u)\right\} E_{\nu}^{\mu}-\operatorname{Im}\left\{H_{\bar{\nu} \mu \lambda}(u)\right\} J_{o} \cdot E_{\nu}^{\mu} \\
E_{2 \lambda+1}(u)= & J_{o} \cdot E_{\lambda}^{0}-\left[\operatorname{Re}\left\{h_{\lambda \mu}(u)\right\}-\delta_{\lambda \mu}\right] J_{o} \cdot E_{0}^{\mu}+\operatorname{Im}\left\{h_{\lambda \mu}(u)\right\} E_{0}^{\mu} \\
& -\operatorname{Re}\left\{H_{\bar{\nu} \mu \lambda}(u)\right\} J_{o} \cdot E_{\nu}^{\mu}+\operatorname{Im}\left\{H_{\bar{\nu} \mu \lambda}(u)\right\} E_{\nu}^{\mu} .
\end{aligned}
$$

Consider also the complex valued vector fields $\tilde{e}_{\lambda}^{\prime}, \tilde{e}_{\bar{\lambda}}^{\prime}$ defined as

$$
\left.\tilde{e}_{\lambda}^{\prime}\right|_{u}=\frac{1}{2}\left\{\left.E_{2 \lambda}(u)^{*}\right|_{u}-\left.\sqrt{-1} E_{2 \lambda+1}(u)^{*}\right|_{u}\right\},\left.\quad \tilde{e}_{\bar{\lambda}}^{\prime}\right|_{u}=\overline{\left.\tilde{e}_{\lambda}^{\prime}\right|_{u}}
$$

If we consider the vector fields $\tilde{e}_{\lambda}^{\prime}$ and the generalized fundamental vector fields $\tilde{E}_{\lambda, \mu}^{R}$, $\tilde{E}_{\lambda, \mu}^{I}, \tilde{t}$ as vector fields of $T^{\mathbb{C}} L^{\mathbb{C}}(M)$, we may write them as linear combinations of the vector fields $\mathcal{E}_{\beta}^{\alpha}$ and $\mathcal{E}_{\bar{\beta}}^{\bar{\alpha}}$. In this way, we obtain the following expressions:

$$
\begin{aligned}
& \tilde{E}_{\lambda, \mu}^{R}=\left(\mathcal{E}_{\mu}^{\lambda}-\mathcal{E}_{\lambda}^{\mu}\right)+\left(\mathcal{E}_{\bar{\mu}}^{\bar{\lambda}}-\mathcal{E}_{\bar{\lambda}}^{\bar{\mu}}\right), \quad \tilde{E}_{\lambda, \mu}^{I}=\sqrt{-1}\left(\mathcal{E}_{\mu}^{\lambda}+\mathcal{E}_{\lambda}^{\mu}\right)-\sqrt{-1}\left(\mathcal{E}_{\bar{\mu}}^{\bar{\lambda}}+\mathcal{E}_{\bar{\lambda}}^{\bar{\mu}}\right) \\
& \tilde{t}=\sqrt{-1} \mathcal{E}_{0}^{0}-\sqrt{-1} \mathcal{E}_{\overline{0}}^{\overline{0}}, \quad \tilde{e}_{\lambda}^{\prime}=\left.\mathcal{E}_{\lambda}^{0}\right|_{u}-\left.\mathcal{E}_{\overline{0}}^{\bar{\lambda}}\right|_{u}-\left.h_{\mu \lambda}(u) \mathcal{E}_{0}^{\mu}\right|_{u}-\left.H_{\bar{\nu} \mu \lambda}(u) \mathcal{E}_{\nu}^{\mu}\right|_{u}
\end{aligned}
$$

Notice that the vectors $E_{\lambda, \mu}^{R}, E_{\lambda, \mu}^{I}$ constitute a basis for $\mathfrak{u}_{n-1}$; hence any complex vector $X \in T_{u}^{\mathbb{C}} \mathbb{V}_{x}$, which is vertical w.r.t. the projection $\hat{\pi}_{\pi(x)}: \mathbb{V}_{\pi(x)} \rightarrow S_{\pi(x)}=$ $\mathbb{V}_{\pi(x)} / U_{n-1}$, is linear combination of the vectors $\tilde{E}_{\lambda, \mu}^{R}(u), \tilde{E}_{\lambda, \mu}^{I}(u)$ and hence, by (5.10), it is of the form

$$
X=C_{\nu}^{\mu}\left(\left.\mathcal{E}_{\mu}^{\nu}\right|_{u}-\left.\mathcal{E}_{\bar{\nu}}^{\bar{\mu}}\right|_{u}\right)
$$

for some uniquely determined $C_{\nu}^{\mu} \in \mathbb{C}$.

At the same time, for any $u \in U_{F}(M)$, the generalized fundamental vector fields $\tilde{t}$ and $\widetilde{E_{i}(u)}, i=2, \ldots, 2 n-1$, span a subspace of $T_{u} \mathbb{V}_{\pi(u)}$, which is of dimension $2 n-1$ and which is complementary to the vertical distribution. More precisely we have the following:

Lemma 5.3. For any $u \in U_{F}(M)$

$$
\begin{aligned}
& \left.\tilde{\epsilon}_{1}\right|_{u}=\left.\tilde{t}\right|_{u} \bmod \operatorname{span}_{\mathbb{R}}\left\{A_{u}^{*}, A \in \mathfrak{u}_{n-1}\right\}, \\
& \left.\tilde{\epsilon}_{2 \lambda}\right|_{u}=\left.\left(E_{2 \lambda}(u)\right)^{*}\right|_{u} \bmod \operatorname{span}_{\mathbb{R}}\left\{A_{u}^{*}, A \in \mathfrak{u}_{n-1}\right\}, \\
& \left.\tilde{\epsilon}_{2 \lambda+1}\right|_{u}=\left.\left(E_{2 \lambda+1}(u)\right)^{*}\right|_{u} \bmod \operatorname{span}_{\mathbb{R}}\left\{A_{u}^{*}, A \in \mathfrak{u}_{n-1}\right\} .
\end{aligned}
$$


Moreover, there exist some complex valued functions $S_{\sigma}^{\rho}$ and $S_{\sigma \lambda}^{\rho}$ such that

$$
\begin{aligned}
& \left.\tilde{\epsilon}_{1}\right|_{u}=\left.\tilde{t}\right|_{u}-S_{\sigma}^{\rho}(u)\left(\left.\mathcal{E}_{\rho}^{\sigma}\right|_{u}-\left.\mathcal{E}_{\bar{\sigma}}^{\bar{\rho}}\right|_{u}\right) \\
& \left.\tilde{e}_{\lambda}\right|_{u}=\left.\mathcal{E}_{\lambda}^{0}\right|_{u}-\left.\mathcal{E}_{\overline{0}}^{\bar{\lambda}}\right|_{u}-\left.h_{\sigma \lambda}(u) \mathcal{E}_{0}^{\sigma}\right|_{u}-\left.H_{\bar{\rho} \sigma \lambda}(u) \mathcal{E}_{\rho}^{\sigma}\right|_{u}-S_{\sigma \lambda}^{\rho}(u)\left(\left.\mathcal{E}_{\rho}^{\sigma}\right|_{u}-\left.\mathcal{E}_{\bar{\sigma}}^{\bar{\rho}}\right|_{u}\right) .
\end{aligned}
$$

Proof. In order to prove the claim, observe that, for any fiber $\mathbb{V}_{x}$ and any frame $u=\left\{f_{0}, \ldots, f_{2 n-1}\right\} \in \mathbb{V}_{x}$, the element $y=[u]=\hat{\pi}_{x}(u) \in \mathbb{V}_{x} / U_{n-1}=S^{2 n-1}$ may be identified with the first element $f_{0}=u\left(\epsilon_{0}\right)$ of the frame. Therefore the vector $\left.t^{*}\right|_{u}$ is projected by $\hat{\pi}_{x}$ onto the vector of $T_{[u]=f_{0}} S_{x}$ given by

$$
\begin{aligned}
\hat{\pi}_{x *}\left(\left.t^{*}\right|_{u}\right) & =\left.\frac{d}{d s}\left(\hat{\pi}_{x}\left(u \circ e^{s J_{o} E_{0}^{0}}\right)\right)\right|_{s=0}=\left.\frac{d}{d s}\left(u \circ e^{s J_{o} E_{0}^{0}}\left(\epsilon_{0}\right)\right)\right|_{s=0} \\
& =u \circ J_{o} E_{0}^{0}\left(\epsilon_{0}\right)=u \circ J_{o} E_{0}^{0}\left(\varepsilon_{0}+\varepsilon_{\overline{0}}\right)=u\left(i \varepsilon_{0}-i \varepsilon_{\overline{0}}\right)=f_{1} .
\end{aligned}
$$

By a similar argument one can check that $\left.\left(E_{2 \lambda}(u)\right)^{*}\right|_{u}$ and $\left(E_{2 \lambda+1}(u)\right)_{u}^{*}$ are mapped by $\hat{\pi}_{x}$ onto the vectors $f_{2 \lambda}, f_{2 \lambda+1}=J f_{2 \lambda}$, respectively. This proves (5.13).

From (5.13) and (5.12), the formulae (5.14) and (5.15) follow.

In the following, in order to have more symmetry in some formulas, we will often write $H_{\overline{0} \sigma \lambda}(u)$ in place of $h_{\sigma \lambda}(u)$, since they coincide by Lemma 5.1 .

5.3. The structure functions of the absolute parallelism of $U_{F}(M)$. Cartan-Sternberg theorem (see [St]; we recall the complete statement - which is indeed quite long - in the Appendix) implies that a complete set of invariant functions for an absolute parallelism $\left\{X_{1}, \ldots, X_{m}\right\}$ is given by the structure functions $c_{j k}^{i}$, defined by $\left[X_{j}, X_{k}\right]=c_{j k}^{i} X_{i}$, and by their derivatives $X_{i_{1}}\left(\ldots X_{i_{p}}\left(c_{j k}^{i}\right) \ldots\right)$, with $p$ less or equal to some finite order $r$. The order $r$ in general depends on the absolute parallelism, but, in case of real analytic data, there exists an upper bound for $r$ which depends only on the dimension of the manifold (this is a consequence of Cartan-Kähler theorem; see [BCG]).

From this remarks and Proposition 4.6 (1), we conclude that the structure functions $c_{j k}^{i}$ and the derivatives $X_{i_{1}}\left(\ldots X_{i_{p}}\left(c_{j k}^{i}\right) \ldots\right)$ of the absolute parallelism (4.3) are a complete system of invariant functions for the complex Finsler manifold $(M, J, F)$.

In this section we want to describe these structure functions.

The structure functions $c_{j k}^{i}$ corresponding to Lie brackets of two generalized fundamental vector fields $\tilde{X}, \tilde{Y}$, with $X$ and $Y$ in $\left\{t, E_{\lambda, \mu}^{R}, E_{\lambda, \mu}^{I}\right\}$, are computed by the Lie brackets in $\mathfrak{u}_{n-1} \oplus \mathbb{R}$. In fact, $\tilde{X}$ and $\tilde{Y}$ are the fundamental vector fields in the usual sense and hence $[\tilde{X}, \tilde{Y}]=\widetilde{[X, Y]}$. In particular, for those Lie brackets, the corresponding structure functions $c_{j k}^{i}$ are the structure constants of the Lie algebra $\mathfrak{u}_{n-1} \oplus \mathbb{R}$.

The structure functions $c_{j k}^{i}$ corresponding to Lie brackets between a fundamental vector fields $\tilde{X}$, with $X \in\left\{E_{\lambda, \mu}^{R}, E_{\lambda, \mu}^{I}\right\}$ and a vector field $Y$ in the set $\left\{\hat{\epsilon}_{i}, \tilde{\epsilon}_{j}\right\}$ can be evaluated recalling that $\tilde{X}$ is a fundamental vector field associated to an element in $\mathfrak{u}_{n-1}$ and that $Y$ belongs either to $\mathcal{H}$ or to $\mathcal{W}$, which are both invariant under the action of $U_{n-1}$. This implies that the action of $\tilde{X}$ on the set $\left\{\hat{\epsilon}_{i}, \tilde{\epsilon}_{j}\right\}$ is equal to the standard action of $X \in \mathfrak{u}_{n-1}$ on the basis $\left\{\epsilon_{i}, \epsilon_{j}\right\}$ of $V \oplus W=\mathbb{C}^{n} \oplus \mathbb{C}^{n-1}$ (see e.g. Prop. 2.3 in $[\mathrm{KN}]$, vol. I). Therefore, also for these Lie brackets, the corresponding structure functions are constant. 
The structure functions corresponding to the Lie brackets of two vector fields in the set $\left\{\hat{\epsilon}_{i}, \tilde{\epsilon}_{j}, \tilde{t}\right\}$ are given by the real and imaginary parts of the Lie brackets described in the following Propositions 5.4, 5.5 and 5.6.

Proposition 5.4. A Lie bracket of a pair of vector fields in the set $\left\{\hat{e}_{\beta}, \hat{e}_{\bar{\gamma}}, \tilde{t}\right\}$ or of a pair in the set $\left\{\tilde{e}_{\lambda}, \tilde{e}_{\bar{\lambda}}, \tilde{t}\right\}$ has one of the following forms:

$$
\begin{aligned}
& {\left[\hat{e}_{\beta}, \hat{e}_{\gamma}\right]=-T_{\beta \gamma}^{\alpha} \hat{e}_{\alpha}} \\
& {\left[\hat{e}_{\beta}, \hat{e}_{\bar{\gamma}}\right]=-R_{0 \beta \bar{\gamma}}^{\lambda} \tilde{e}_{\lambda}+R_{\lambda \beta \bar{\gamma}}^{0} \tilde{e}_{\bar{\lambda}}+\sqrt{-1} R_{0 \beta \bar{\gamma}}^{0} \tilde{t}-R_{\sigma \beta \bar{\gamma}}^{\rho}\left(\mathcal{E}_{\rho}^{\sigma}-\mathcal{E}_{\bar{\rho}}^{\bar{\sigma}}\right),} \\
& {\left[\tilde{t}, \hat{e}_{\beta}\right]=\sqrt{-1} \delta_{\beta 0} \hat{e}_{0}, \quad\left[\tilde{t}, \hat{e}_{\bar{\beta}}\right]=-\sqrt{-1} \delta_{\beta 0} \hat{e}_{\overline{0}}} \\
& {\left[\tilde{e}_{\mu}, \tilde{e}_{\nu}\right]=0, \quad\left[\tilde{e}_{\mu}, \tilde{e}_{\bar{\nu}}\right]=-\sqrt{-1} \delta_{\mu \nu} \tilde{t}-\left(\delta_{\mu}^{\rho} \delta_{\sigma \nu}-Q_{\sigma \mu \bar{\nu}}^{\rho}\right)\left(\mathcal{E}_{\rho}^{\sigma}-\mathcal{E}_{\bar{\sigma}}^{\bar{\rho}}\right)} \\
& {\left[\tilde{t}, \tilde{e}_{\nu}\right]_{u}=-\sqrt{-1} \tilde{e}_{\nu}-\sqrt{-1} Q_{\sigma 0 \nu}^{\rho}\left(\mathcal{E}_{\rho}^{\sigma}-\mathcal{E}_{\bar{\sigma}}^{\bar{\rho}}\right)} \\
& {\left[\tilde{t}, \tilde{e}_{\bar{\nu}}\right]=\sqrt{-1} \tilde{e}_{\bar{\nu}}-\sqrt{-1 Q_{\rho 0 \nu}^{\sigma}}\left(\mathcal{E}_{\rho}^{\sigma}-\mathcal{E}_{\bar{\sigma}}^{\bar{\rho}}\right)}
\end{aligned}
$$

where $T_{\beta \gamma}^{\alpha}, R_{\beta \gamma \bar{\delta}}^{\alpha}, Q_{\sigma \mu \bar{\nu}}^{\rho}, Q_{\sigma 0 \nu}^{\rho}$ and $Q_{\bar{\sigma} 0 \nu}^{\bar{\rho}}$ are some uniquely determined $\mathbb{C}$-valued functions.

Proof. Recall that the vector fields $\hat{e}_{\alpha}$ are holomorphic vector fields in $\mathcal{H}^{10} \subset \mathcal{H}^{\mathbb{C}}$. Since the CR structure $(\mathcal{H}, \hat{J})$ is integrable, $\left[\hat{e}_{\beta}, \hat{e}_{\gamma}\right]$ takes values in $\mathcal{H}^{10}$. From this (5.16) follows.

To prove (5.17), we first claim that it is a complex vertical vector. In fact, for a given $u \in U_{F}(M)$, consider a local holomorphic embedding $\sigma: \mathcal{U} \subset M \rightarrow U_{F}(M)$ with $u \in \sigma(\mathcal{U})$. Since $\pi: L^{\mathbb{C}}(M) \rightarrow M$ is holomorphic, $\hat{e}_{\beta}^{\prime}=(\sigma \circ \pi)_{*}\left(\left.\hat{e}_{\beta}\right|_{\sigma(\mathcal{U})}\right)$ and $\hat{e}_{\bar{\gamma}}^{\prime}=(\sigma \circ \pi)_{*}\left(\left.\hat{e}_{\bar{\gamma}}\right|_{\sigma(\mathcal{U})}\right)$ are holomorphic and antiholomorphic vector fields on $\sigma(\mathcal{U})$, respectively, and hence $\left[\hat{e}_{\beta}^{\prime}, \hat{e}_{\bar{\gamma}}^{\prime}\right]=0$. Since at any $u^{\prime}=\left.A \cdot \sigma(x) \in U_{F}(M)\right|_{\mathcal{U}}$ we may write $\hat{e}_{\beta}$ and $\hat{e}_{\bar{\gamma}}$ in the form $\hat{e}_{\beta}\left(u^{\prime}\right)=\left.A_{\beta}^{\delta} \cdot \hat{e}_{\delta}^{\prime}\right|_{\sigma(x)}+\left.\Gamma_{\varepsilon \beta}^{\delta}\left(u^{\prime}\right)\left(E_{\delta}^{\varepsilon}\right)^{*}\right|_{u^{\prime}}$ and $\hat{e}_{\bar{\gamma}}=\left.A_{\bar{\gamma}}^{\bar{\delta}} \cdot \hat{e}_{\bar{\delta}}^{\prime}\right|_{\sigma(u)}+\left.\Gamma_{\bar{\varepsilon} \bar{\gamma}}^{\bar{\delta}}\left(u^{\prime}\right)\left(E_{\bar{\delta}}^{\bar{\varepsilon}}\right)^{*}\right|_{u^{\prime}}$, a simple computation shows that $\pi_{*}\left(\left[e_{\beta}, e_{\bar{\gamma}}\right]_{u}\right)=0$, i.e. that it is a complex vertical vector. Since at any $u$, the vectors $\left.\tilde{e}_{\lambda}\right|_{u},\left.\tilde{e}_{\bar{\lambda}}\right|_{u}, \sqrt{-1} \tilde{t}_{u}$ and $\left.\mathcal{E}_{\rho}^{\sigma}\right|_{u}-\left.\mathcal{E}_{\bar{\sigma}}^{\bar{\rho}}\right|_{u}$ are linearly independent over $\mathbb{C}$ and the complexified vertical subspace $\mathcal{V}_{u}^{\mathbb{C}} \subset T_{u}^{\mathbb{C}} U_{F}(M)$ is equal to their span, (5.17) follows.

To check (5.18), recall that $\tilde{t}$ is the fundamental vector field in $L^{\mathbb{C}}(M)$ associated to $J_{o} \cdot E_{0}^{0}$; then the formula follows from definitions and the fact that $\mathcal{H}$ is $U_{n-1} \times T^{1}$ invariant.

(5.19) is a consequence of the properties of the distribution $\mathcal{W}$ (see Definition $4.3)$, of (5.14) and (5.15).

The proof of (5.20) is the following. Pick a frame $u_{o} \in U_{F}(M)$ and let $X_{\nu}$ the complex vector field in $T^{\mathbb{C}} L^{\mathbb{C}}(M)$ defined by

$$
X_{\nu}=\mathcal{E}_{\nu}^{0}-\mathcal{E}_{\overline{0}}^{\bar{\nu}}-H_{\bar{\alpha} \sigma \nu}\left(u_{o}\right) \mathcal{E}_{\alpha}^{\sigma}-S_{\sigma \lambda}^{\rho}\left(u_{o}\right)\left(\mathcal{E}_{\rho}^{\sigma}-\mathcal{E}_{\bar{\sigma}}^{\bar{\rho}}\right) .
$$

From definitions and Lemma 5.3, one can check that

$$
\left[\tilde{t}, \tilde{e}_{\nu}\right]_{u_{o}}=\left[\tilde{t}, X_{\nu}\right]_{u_{o}} \bmod \operatorname{span}_{\mathbb{C}}\left\{\left.\mathcal{E}_{0}^{\sigma}\right|_{u_{o}},\left.\mathcal{E}_{\rho}^{\sigma}\right|_{u_{o}},\left.\mathcal{E}_{\bar{\rho}}^{\bar{\sigma}}\right|_{u_{o}}\right\}
$$

By the properties of the Lie brackets between fundamental vector fields in $L^{\mathbb{C}}(M)$, it follows that there exists some complex functions $Q_{B C D}^{A}$ such that

$$
\left[\tilde{t}, \tilde{e}_{\nu}\right]=-\sqrt{-1} \tilde{e}_{\nu}-\sqrt{-1} Q_{\sigma 0 \nu}^{0} \mathcal{E}_{0}^{\sigma}-\sqrt{-1} Q_{\sigma 0 \nu}^{\rho} \mathcal{E}_{\rho}^{\sigma}-\sqrt{-1} Q_{\bar{\sigma} 0 \nu}^{\bar{\rho}} \mathcal{E}_{\bar{\rho}}^{\bar{\sigma}}
$$


Notice that by (5.10) and (5.11) the vector

$$
\left[\tilde{t}, \tilde{e}_{\nu}\right]_{u}+\left.\sqrt{-1} \tilde{e}_{\nu}\right|_{u}=-\left.\sqrt{-1} Q_{\sigma 0 \nu}^{0}(u) \mathcal{E}_{0}^{\sigma}\right|_{u}-\sqrt{-1} Q_{\sigma 0 \nu}^{\rho}(u) \mathcal{E}_{\rho}^{\sigma}-\left.\left.\sqrt{-1}\right|_{u} Q_{\bar{\sigma} 0 \nu}^{\bar{\rho}}(u) \mathcal{E}_{\bar{\rho}}^{\bar{\sigma}}\right|_{u}
$$

belongs to $\operatorname{span}_{\mathbb{C}}\left\{\left.\tilde{e}_{\lambda}\right|_{u},\left.\tilde{e}_{\bar{\lambda}}\right|_{u},\left.\tilde{t}\right|_{u},\left.\mathcal{E}_{\rho}^{\sigma}\right|_{u}-\left.\mathcal{E}_{\bar{\sigma}}^{\bar{\rho}}\right|_{u}\right\}$ only if $Q_{\sigma 0 \nu}^{0}(u)=0$ and $Q_{\bar{\sigma} 0 \nu}^{\bar{\rho}}(u)=$ $Q_{\rho 0 \nu}^{\sigma}(u)$. This concludes the proof of (5.20). (5.21) is obtained from (5.20) by conjugation and taking into account the fact that $\overline{\tilde{t}}=\tilde{t}$.

Proposition 5.5. At any frame $u \in U_{F}(M)$ and for any $\rho, \sigma, \lambda, \mu=1, \ldots, n-$ 1 ,

(1) $S_{\sigma \lambda}^{\rho}(u)=0$; in particular $\tilde{e}_{\lambda}^{\prime} \equiv \tilde{e}_{\lambda}$;

(2) $Q_{\sigma 0 \mu}^{\rho \lambda}(u)=0$ and

$$
Q_{\sigma \lambda \bar{\mu}}^{\rho}(u)=\left.\tilde{e}_{\bar{\mu}}\left(H_{\sigma \lambda \bar{\rho}}\right)\right|_{u}=H_{\bar{\mu} \bar{\rho} \sigma \lambda}(u)-h_{\bar{\mu} \bar{\rho}}(u) h_{\sigma \lambda}(u)-H_{\nu \bar{\mu} \bar{\rho}}(u) H_{\sigma \lambda \bar{\nu}}(u) ;
$$

(3) the Lie brackets $\left[\tilde{e}_{\lambda}, \hat{e}_{0}\right],\left[\tilde{e}_{\bar{\lambda}}, \hat{e}_{0}\right],\left[\tilde{e}_{\lambda}, \hat{e}_{\mu}\right],\left[\tilde{e}_{\bar{\lambda}}, \hat{e}_{\mu}\right]$ have the following forms:

$$
\begin{aligned}
& {\left[\tilde{e}_{\lambda}, \hat{e}_{0}\right]=\hat{e}_{\lambda}+\sqrt{-1} P_{0 \lambda 0}^{0} \tilde{t}-P_{0 \lambda 0}^{\mu} \tilde{e}_{\mu}+P_{\mu \lambda 0}^{0} \tilde{e}_{\bar{\mu}}-P_{\rho \lambda 0}^{\sigma}\left(\mathcal{E}_{\sigma}^{\rho}-\mathcal{E}_{\bar{\rho}}^{\bar{\sigma}}\right)} \\
& {\left[\tilde{e}_{\lambda}, \hat{e}_{\mu}\right]=-H_{\bar{\alpha} \mu \lambda} \hat{e}_{\alpha}+\sqrt{-1} P_{0 \lambda \mu}^{0} \tilde{t}-P_{0 \lambda \mu}^{\nu} \tilde{e}_{\nu}+P_{\nu \lambda \mu}^{0} \tilde{e}_{\bar{\nu}}-P_{\rho \lambda \mu}^{\sigma}\left(\mathcal{E}_{\sigma}^{\rho}-\mathcal{E}_{\bar{\rho}}^{\bar{\sigma}}\right)} \\
& {\left[\tilde{e}_{\bar{\lambda}}, \hat{e}_{0}\right]=-\sqrt{-1} P_{0 \bar{\lambda} 0}^{0} \tilde{t}+P_{0 \bar{\lambda} 0}^{\mu} \tilde{e}_{\mu}-P_{\mu \bar{\lambda} 0}^{0} \tilde{e}_{\bar{\mu}}+P_{\rho \bar{\lambda} 0}^{\sigma}\left(\mathcal{E}_{\sigma}^{\rho}-\mathcal{E}_{\bar{\rho}}^{\bar{\sigma}}\right)} \\
& {\left[\tilde{e}_{\bar{\lambda}}, \hat{e}_{\mu}\right]=-\delta_{\lambda \mu} \hat{e}_{0}-\sqrt{-1} P_{0 \bar{\lambda} \mu}^{0} \tilde{t}+P_{0 \bar{\lambda} \mu}^{\nu} \tilde{e}_{\nu}-P_{\nu \bar{\lambda} \mu}^{0} \tilde{e}_{\bar{\nu}}+P_{\rho \bar{\lambda} \mu}^{\sigma}\left(\mathcal{E}_{\sigma}^{\rho}-\mathcal{E}_{\bar{\rho}}^{\bar{\sigma}}\right)}
\end{aligned}
$$

where $P_{B C D}^{A}$ are some uniquely determined complex valued functions.

Proof. (1) For any frame $u$, let us denote the vertical subspace of $U_{F}(M)$ by $\mathcal{V}_{u}$. We claim that

$$
\begin{aligned}
& {\left[\tilde{e}_{\lambda}, \hat{e}_{0}\right]_{u}=\hat{e}_{\lambda} \bmod \mathcal{V}_{u}^{\mathbb{C}}, \quad\left[\tilde{e}_{\lambda}, \hat{e}_{\mu}\right]_{u}=-\left(H_{\bar{\alpha} \mu \lambda}+S_{\mu \lambda}^{\alpha}\right) \hat{e}_{\alpha} \bmod \mathcal{V}_{u}^{\mathbb{C}},} \\
& {\left[\tilde{e}_{\bar{\lambda}}, \hat{e}_{0}\right]_{u}=0 \bmod \mathcal{V}_{u}^{\mathbb{C}}, \quad\left[\tilde{e}_{\bar{\lambda}}, \hat{e}_{\mu}\right]=-\delta_{\lambda \mu} \hat{e}_{0}+\overline{S_{\nu \lambda}^{\mu}} \hat{e}_{\nu} \bmod \mathcal{V}_{u}^{\mathbb{C}},}
\end{aligned}
$$

where $S_{\rho \lambda}^{\sigma}$ and $S_{\bar{\rho} \lambda}^{\bar{\sigma}}$ are the complex functions defined in Lemma 5.3 and we let $S_{\rho \lambda}^{0}=0$.

To prove (5.26), let us fix a frame $u_{o}$ and consider the complex vector field $X_{\lambda}$ in $T^{\mathbb{C}} L^{\mathbb{C}}(M)$ defined by

$$
X_{\lambda}=\mathcal{E}_{\lambda}^{0}-\mathcal{E}_{\overline{0}}^{\bar{\lambda}}-\left(H_{\bar{\alpha} \sigma \lambda}\left(u_{o}\right)+S_{\sigma \lambda}^{\alpha}\left(u_{o}\right)\right) \mathcal{E}_{\alpha}^{\sigma}+S_{\rho \lambda}^{\sigma}\left(u_{o}\right) \mathcal{E}_{\bar{\rho}}^{\bar{\sigma}}
$$

Let us also extend $\hat{e}_{0}$ to a vector field on a neighborhood $\mathcal{U} \subset L^{\mathbb{C}}(M)$ of $u_{o}$.

From (5.15) and from definitions we get

$$
\left[\tilde{e}_{\lambda}, \hat{e}_{0}\right]_{u_{o}}=\left[X_{\lambda}, \hat{e}_{0}\right]_{u_{o}} \bmod \operatorname{span}_{\mathbb{C}}\left\{\left.\mathcal{E}_{\alpha}^{\sigma}\right|_{u_{o}},\left.\mathcal{E}_{\bar{\rho}}^{\bar{\sigma}}\right|_{u_{o}}\right\}
$$

In particular, $\theta_{u_{o}}\left(\left[\tilde{e}_{\lambda}, \hat{e}_{0}\right]\right)=\theta_{u_{o}}\left(\left[X_{\lambda}, \hat{e}_{0}\right]\right)$. Moreover, $\left.X_{\lambda}\left(\theta\left(\hat{e}_{0}\right)\right)\right|_{u_{o}}=0$ : in fact $\left.X_{\lambda}\right|_{u_{o}}=\left.\tilde{e}_{\lambda}\right|_{u_{o}}$ and hence it is tangent to $U_{F}(M)$; on the other hand $\theta\left(\hat{e}_{0}\right)=e_{0}$ at all points of $U_{F}(M)$.

Therefore

$$
\begin{aligned}
& \theta_{u_{o}}\left(\left[X_{\lambda}, \hat{e}_{0}\right]\right)=-\left(\mathcal{L}_{X_{\lambda}} \theta\right)_{u_{o}}\left(\hat{e}_{0}\right)=-\left(\mathcal{L}_{\mathcal{E}_{\lambda}^{0}-\mathcal{E}_{\overline{\hat{\alpha}}}^{\bar{\lambda}}} \theta\right)_{u_{o}}\left(\hat{e}_{0}\right) \\
& \quad+\left(H_{\bar{\alpha} \rho \lambda}\left(u_{o}\right)+S_{\rho \lambda}^{\alpha}\left(u_{o}\right)\right)\left(\mathcal{L}_{\mathcal{E}_{\alpha}^{\rho} \theta} \theta\right)_{u_{o}}\left(\hat{e}_{0}\right)-S_{\sigma \lambda}^{\rho}\left(u_{o}\right)\left(\mathcal{L}_{\mathcal{E}_{\bar{\sigma}}^{\bar{\rho}}} \theta\right)_{u_{o}}\left(\hat{e}_{0}\right)=e_{\lambda} .
\end{aligned}
$$

This implies that at all points the vectors $\left[\tilde{e}_{\lambda}, \hat{e}_{0}\right]$ and $\hat{e}_{\lambda}$ differ by a complex vertical vector. This implies the first identity in (5.26). The second identity in (5.26) and the two identities of (5.27) are proved with the same arguments. 
Now, we can prove that $S_{\mu \nu}^{\lambda}=0$. Indeed from the Jacobi identities, Proposition $5.4,(5.26)$ and $(5.27)$

$$
0=\theta^{\mu}\left(\left[\tilde{e}_{\lambda},\left[\tilde{e}_{\bar{\nu}}, \hat{e}_{0}\right]\right]\right)+\theta^{\mu}\left(\left[\tilde{e}_{\bar{\nu}},\left[\hat{e}_{0}, \tilde{e}_{\lambda}\right]\right]\right)+\theta^{\mu}\left(\left[\hat{e}_{0},\left[\tilde{e}_{\lambda}, \tilde{e}_{\bar{\nu}}\right]\right]\right)=\theta^{\mu}\left(\left[\tilde{e}_{\bar{\nu}},\left[\hat{e}_{0}, \tilde{e}_{\lambda}\right]\right]\right)=-\overline{S_{\mu \nu}^{\lambda}}
$$

(2) is a consequence of (1), (5.26), (5.27), the Jacobi identities and Lemma 2.4. In fact,

$$
\begin{aligned}
0 & =\theta^{\rho}\left(\left[\tilde{e}_{\lambda},\left[\tilde{e}_{\bar{\mu}}, \hat{e}_{\sigma}\right]\right]\right)+\theta^{\rho}\left(\left[\tilde{e}_{\bar{\mu}},\left[\hat{e}_{\sigma}, \tilde{e}_{\lambda}\right]\right]\right)+\theta^{\rho}\left(\left[\hat{e}_{\sigma},\left[\tilde{e}_{\lambda}, \tilde{e}_{\bar{\mu}}\right]\right]\right) \\
& =\theta^{\rho}\left(\left[\tilde{e}_{\lambda},\left[\tilde{e}_{\bar{\mu}}, \hat{e}_{\sigma}\right]\right]\right)+\theta^{\rho}\left(\left[\tilde{e}_{\bar{\mu}},\left[\hat{e}_{\sigma}, \tilde{e}_{\lambda}\right]\right]\right)+\delta_{\lambda}^{\rho} \delta_{\sigma \mu}-Q_{\sigma \lambda \bar{\mu}}^{\rho} \\
& =-\delta_{\mu \sigma} \delta_{\lambda}^{\rho}+\tilde{e}_{\bar{\mu}}\left(H_{\bar{\rho} \sigma \lambda}\right)+\delta_{\lambda}^{\rho} \delta_{\sigma \mu}-Q_{\sigma \lambda \bar{\mu}}^{\rho}
\end{aligned}
$$

and

$$
0=\theta^{\rho}\left(\left[\tilde{t},\left[\tilde{e}_{\bar{\mu}}, \hat{e}_{\sigma}\right]\right]\right)+\theta^{\rho}\left(\left[\tilde{e}_{\bar{\mu}},\left[\hat{e}_{\sigma}, \tilde{t}\right]\right]\right)+\theta^{\rho}\left(\left[\hat{e}_{\sigma},\left[\tilde{t}, \tilde{e}_{\bar{\mu}}\right]\right]\right)=\sqrt{-1} \overline{Q_{\rho 0 \mu}^{\sigma}} .
$$

Now, using Lemma $2.4,(5.15)$ and the fact that $S_{\sigma \lambda}^{\rho}=0$, a straightforward computation shows that

$$
\tilde{e}_{\bar{\mu}}\left(H_{\bar{\rho} \sigma \lambda}\right)=-h_{\bar{\mu} \bar{\rho}} h_{\sigma \lambda}-H_{\nu \bar{\mu} \bar{\rho}} H_{\sigma \lambda \bar{\nu}}+H_{\bar{\mu} \bar{\rho} \sigma \lambda}
$$

and this concludes the proof.

(3) is an immediate consequence of (5.26), (5.27) and of claim (1).

Proposition 5.6. The structure functions $P_{B C D}^{A}$ defined in Proposition 5.5 are the following:

(1) $P_{0 \rho \gamma}^{0}=P_{0 \bar{\rho} \gamma}^{0}=P_{\lambda \rho \gamma}^{0}=P_{\lambda \bar{\rho} \gamma}^{0}=P_{0 \rho \gamma}^{\lambda}=P_{\mu \rho \gamma}^{\lambda}=0$;

(2) $P_{0 \bar{\rho} \gamma}^{\lambda}=-\hat{e}_{\gamma}\left(h_{\bar{\lambda} \bar{\rho}}\right), P_{\mu \bar{\rho} \gamma}^{\lambda}=-\hat{e}_{\gamma}\left(H_{\bar{\lambda} \mu \bar{\rho}}\right)$.

Proof. It suffices to use the Jacobi identities, Proposition 5.4 and Proposition 5.5. In fact

$$
\begin{aligned}
0 & =\theta^{\overline{0}}\left(\left[\tilde{e}_{\rho},\left[\hat{e}_{\overline{0}}, \hat{e}_{\gamma}\right]\right]\right)+\theta^{\overline{0}}\left(\left[\hat{e}_{\gamma},\left[\tilde{e}_{\rho}, \hat{e}_{\overline{0}}\right]\right]\right)+\theta^{\overline{0}}\left(\left[\hat{e}_{\overline{0}},\left[\hat{e}_{\gamma}, \tilde{e}_{\rho}\right]\right]\right)=P_{0 \rho \gamma}^{0}, \\
0 & =\theta^{\overline{0}}\left(\left[\tilde{e}_{\bar{\rho}},\left[\hat{e}_{\overline{0}}, \hat{e}_{\gamma}\right]\right]\right)+\theta^{\overline{0}}\left(\left[\hat{e}_{\gamma},\left[\tilde{e}_{\bar{\rho}}, \hat{e}_{\overline{0}}\right]\right]\right)+\theta^{\overline{0}}\left(\left[\hat{e}_{\overline{0}},\left[\hat{e}_{\gamma}, \tilde{e}_{\bar{\rho}}\right]\right]\right)=-P_{0 \bar{\rho} \gamma}^{0}, \\
0 & =\theta^{\bar{\lambda}}\left(\left[\tilde{e}_{\rho},\left[\hat{e}_{\overline{0}}, \hat{e}_{\gamma}\right]\right]\right)+\theta^{\bar{\lambda}}\left(\left[\hat{e}_{\gamma},\left[\tilde{e}_{\rho}, \hat{e}_{\overline{0}}\right]\right]\right)+\theta^{\bar{\lambda}}\left(\left[\hat{e}_{\overline{0}},\left[\hat{e}_{\gamma}, \tilde{e}_{\rho}\right]\right]\right)=P_{\lambda \rho \gamma}^{0}, \\
0 & =\theta^{\bar{\lambda}}\left(\left[\tilde{e}_{\bar{\rho}},\left[\hat{e}_{\overline{0}}, \hat{e}_{\gamma}\right]\right]\right)+\theta^{\bar{\lambda}}\left(\left[\hat{e}_{\gamma},\left[\tilde{e}_{\bar{\rho}}, \hat{e}_{\overline{0}}\right]\right]\right)+\theta^{\bar{\lambda}}\left(\left[\hat{e}_{\overline{0}},\left[\hat{e}_{\gamma}, \tilde{e}_{\bar{\rho}}\right]\right]\right)=-P_{\lambda \bar{\rho} \gamma}^{0}, \\
0 & =\theta^{\overline{0}}\left(\left[\tilde{e}_{\rho},\left[\hat{e}_{\bar{\lambda}}, \hat{e}_{\gamma}\right]\right]\right)+\theta^{\overline{0}}\left(\left[\hat{e}_{\gamma},\left[\tilde{e}_{\rho}, \hat{e}_{\bar{\lambda}}\right]\right]\right)+\theta^{\overline{0}}\left(\left[\hat{e}_{\bar{\lambda}},\left[\hat{e}_{\gamma}, \tilde{e}_{\rho}\right]\right]\right) \\
& =P_{0 \rho \gamma}^{\lambda}-\overline{H_{\overline{0} \lambda \sigma}} P_{\sigma \rho \gamma}^{0}=P_{0 \rho \gamma}^{\lambda}, \\
0 & =\theta^{\bar{\mu}}\left(\left[\tilde{e}_{\rho},\left[\hat{e}_{\bar{\lambda}}, \hat{e}_{\gamma}\right]\right]\right)+\theta^{\bar{\mu}}\left(\left[\hat{e}_{\gamma},\left[\tilde{e}_{\rho}, \hat{e}_{\bar{\lambda}}\right]\right]\right)+\theta^{\bar{\mu}}\left(\left[\hat{e}_{\bar{\lambda}},\left[\hat{e}_{\gamma}, \tilde{e}_{\rho}\right]\right]\right)=-P_{\mu \rho \gamma}^{\lambda}, \\
0 & =\theta^{\overline{0}}\left(\left[\tilde{e}_{\bar{\rho}},\left[\hat{e}_{\bar{\lambda}}, \hat{e}_{\gamma}\right]\right]\right)+\theta^{\overline{0}}\left(\left[\hat{e}_{\gamma},\left[\tilde{e}_{\bar{\rho}}, \hat{e}_{\bar{\lambda}}\right]\right]\right)+\theta^{\overline{0}}\left(\left[\hat{e}_{\bar{\lambda}},\left[\hat{e}_{\gamma}, \tilde{e}_{\bar{\rho}}\right]\right]\right) \\
& =-\hat{e}_{\gamma}\left(\bar{H}_{\overline{0} \lambda \rho}\right)-P_{0 \bar{\rho} \gamma}^{\lambda}=-\hat{e}_{\gamma}\left(h_{\bar{\lambda} \bar{\rho}}\right)-P_{0 \bar{\rho} \gamma}^{\lambda}, \\
0 & =\theta^{\bar{\mu}}\left(\left[\tilde{e}_{\bar{\rho}},\left[\hat{e}_{\bar{\lambda}}, \hat{e}_{\gamma}\right]\right]\right)+\theta^{\bar{\mu}}\left(\left[\hat{e}_{\gamma},\left[\tilde{e}_{\bar{\rho}}, \hat{e}_{\bar{\lambda}}\right]\right]\right)+\theta^{\bar{\mu}}\left(\left[\hat{e}_{\bar{\lambda}},\left[\hat{e}_{\gamma}, \tilde{e}_{\bar{\rho}}\right]\right]\right) \\
& =-\hat{e}_{\gamma}\left(\bar{H}_{\bar{\mu} \lambda \rho}\right)-P_{\mu \bar{\rho} \gamma}^{\lambda}=-\hat{e}_{\gamma}\left(H_{\mu \bar{\lambda} \bar{\rho}}\right)-P_{\mu \bar{\rho} \gamma}^{\lambda} . \quad \square
\end{aligned}
$$

By the previous remarks and Propositions 5.4, 5.5 and 5.6, we now have the complete list for the structure functions of $\sigma^{\mathcal{H}}$ and they generate a complete system of invariant functions. We summarize the results in the next corollary. For notation and indexing conventions, see $\S 5.1$. 
COROLlary 5.7. The structure functions $c_{j k}^{i}$ of the absolute parallelism $\sigma^{\mathcal{H}}$, associated to the non-linear Hermitian connection of a complex Finsler manifold $(M, J, F)$, are the following:

i) the structure constants of $\left(\mathfrak{u}_{n-1}+\mathbb{R}\right) \ltimes \mathbb{C}^{n}$, where the action of $\left(\mathfrak{u}_{n-1}+\mathbb{R}\right)$ on $\mathbb{C}^{n}$ is the one induced by the standard action of $\mathfrak{u}_{n}$ on $\mathbb{C}^{n}$;

ii) the real and the imaginary parts of the functions $h_{\lambda \mu}$ and $H_{\lambda \mu \bar{\nu}}$;

iii) the real and the imaginary parts of the functions

$$
P_{0 \bar{\rho} \gamma}^{\lambda}=-\hat{e}_{\gamma}\left(h_{\bar{\lambda} \bar{\rho}}\right), \quad P_{\mu \bar{\rho} \gamma}^{\lambda}=-\hat{e}_{\gamma}\left(H_{\bar{\lambda} \mu \bar{\rho}}\right), \quad Q_{\sigma \lambda \bar{\mu}}^{\rho}=H_{\bar{\mu} \bar{\rho} \sigma \lambda}-h_{\bar{\mu} \bar{\rho}} h_{\sigma \lambda}-H_{\nu \bar{\mu} \bar{\rho}} H_{\sigma \lambda \bar{\nu}}
$$

iv) the real and the imaginary parts of the functions $T_{\beta \gamma}^{\alpha}$ and $R_{\beta \gamma \bar{\delta}}^{\alpha}$ defined by (5.16) and (5.17).

5.4. The structure equations of a complex Finsler manifold. The structure equations of $U_{F}(M)$ consist in the identities verified by the tautological 1-form $\theta$, the connection form $\omega$ and the differentials of their components. They are direct consequences of the defining equations of the algebraic vertical subspaces and the structure functions of the absolute parallelism. The results are in the following theorem.

For notation and indexing conventions, see $\S 5.1$ and $§ 5.2$.

Theorem 5.8. Let $(M, J, F)$ be a complex Finsler space and let $\omega$ the connection 1-form on $U_{F}(M)$ associated with the non-linear Hermitian connection $\mathcal{H}$ of $(M, J, F)$.

i) The holomorphic and anti-holomorphic components of $\omega$ verify:

$$
\omega_{0}^{0}+\omega_{\overline{0}}^{\overline{0}}=0, \quad \omega_{\lambda}^{0}+\omega_{\overline{0}}^{\bar{\lambda}}+h_{\lambda \nu} \omega_{0}^{\nu}=0, \quad \omega_{\mu}^{\lambda}+\omega_{\bar{\lambda}}^{\bar{\mu}}+H_{\bar{\lambda} \mu \nu} \omega_{0}^{\nu}+H_{\bar{\lambda} \mu \bar{\nu}} \omega_{\overline{0}}^{\bar{\nu}}=0
$$

ii) Let $\varpi_{\beta}^{\alpha}$ be the $\mathbb{C}$-valued 1-forms on $U_{F}(M)$ given by

$$
\varpi_{0}^{0}=\omega_{0}^{0}, \quad \varpi_{0}^{\lambda}=\omega_{0}^{\lambda}, \quad \varpi_{\lambda}^{0}=-\omega_{\overline{0}}^{\bar{\lambda}}, \quad \varpi_{\nu}^{\mu}=\omega_{\nu}^{\mu}+H_{\bar{\mu} \nu \lambda} \omega_{0}^{\lambda}, \quad \varpi_{\bar{\beta}}^{\bar{\alpha}}=\overline{\varpi_{\beta}^{\alpha}} .
$$

Then $\varpi_{\beta}^{\alpha}$ verify:

(5.30) $\varpi_{\bar{\beta}}^{\bar{\alpha}}=-\varpi_{\alpha}^{\beta}$,

(5.31) $\varpi_{0}^{\lambda}\left(\tilde{e}_{\mu}\right)=\delta_{\lambda \mu}, \varpi_{0}^{\lambda}\left(\tilde{e}_{\bar{\mu}}\right)=0, \varpi_{\lambda}^{0}\left(\tilde{e}_{\mu}\right)=0, \varpi_{\lambda}^{0}\left(\tilde{e}_{\bar{\mu}}\right)=-\delta_{\lambda \mu}$,

(5.32) $\varpi_{\lambda}^{0}(\tilde{t})=\varpi_{\lambda}^{0}\left(\tilde{E}_{\mu, \nu}^{R}\right)=\varpi_{\lambda}^{0}\left(\tilde{E}_{\mu, \nu}^{I}\right)=0, \varpi_{0}^{\lambda}(\tilde{t})=\varpi_{0}^{\lambda}\left(\tilde{E}_{\mu, \nu}^{R}\right)=\varpi_{0}^{\lambda}\left(\tilde{E}_{\mu, \nu}^{I}\right)=0$;

iii) The differentials of the tautological 1-form $\theta$ and of the $\mathbb{C}$-valued 1-forms $\varpi_{\gamma}^{\beta}$ are given by the following identities:

$$
\begin{aligned}
& d \theta^{\alpha}+\varpi_{\beta}^{\alpha} \wedge \theta^{\beta}=\Theta^{\alpha}+\Sigma^{\alpha} \\
& d \varpi_{0}^{0}+\varpi_{\beta}^{0} \wedge \varpi_{0}^{\beta}=\Omega_{0}^{0} ; \\
& d \varpi_{0}^{\lambda}+\varpi_{\beta}^{\lambda} \wedge \varpi_{0}^{\beta}=\Omega_{0}^{\lambda}+\Pi_{0}^{\lambda}, \quad d \varpi_{\lambda}^{0}+\varpi_{\beta}^{0} \wedge \varpi_{\lambda}^{\beta}=\Omega_{\lambda}^{0}+\Pi_{\lambda}^{0} \\
& d \varpi_{\mu}^{\lambda}+\varpi_{\beta}^{\lambda} \wedge \varpi_{\mu}^{\beta}=\Omega_{\mu}^{\lambda}+\Pi_{\mu}^{\lambda}+\Phi_{\mu}^{\lambda}
\end{aligned}
$$


where $\Theta^{\alpha}, \Sigma^{\alpha}, \Omega_{\beta}^{\alpha}, \Pi_{0}^{\lambda}, \Pi_{\mu}^{0}, \Pi_{\mu}^{\lambda}$ and $\Phi_{\mu}^{\lambda}$ are the the following $\mathbb{C}$-valued 2-forms:

$$
\begin{aligned}
& \Theta^{\alpha}=\frac{1}{2} T_{\beta \gamma}^{\alpha} \theta^{\beta} \wedge \theta^{\gamma}, \quad \Sigma^{\alpha}=H_{\bar{\alpha} \mu \lambda} \varpi_{0}^{\lambda} \wedge \theta^{\mu}, \quad \Omega_{\beta}^{\alpha}=R_{\beta \gamma \bar{\delta}}^{\alpha} \theta^{\gamma} \wedge \theta^{\bar{\delta}}, \\
& \Pi_{\lambda}^{0}=-\hat{e}_{\bar{\gamma}}\left(h_{\lambda \rho}\right) \varpi_{0}^{\rho} \wedge \theta^{\bar{\gamma}}, \quad \Pi_{0}^{\lambda}=-\hat{e}_{\gamma}\left(h_{\bar{\lambda} \bar{\rho}}\right) \varpi_{\rho}^{0} \wedge \theta^{\gamma}, \\
& \Pi_{\mu}^{\lambda}=-\hat{e}_{\gamma}\left(H_{\bar{\lambda} \mu \bar{\rho}}\right) \varpi_{\rho}^{0} \wedge \theta^{\gamma}-\hat{e}_{\bar{\gamma}}\left(H_{\bar{\lambda} \mu \rho}\right) \varpi_{0}^{\rho} \wedge \theta^{\bar{\gamma}} \\
& \Phi_{\mu}^{\lambda}=\left(H_{\bar{\lambda} \bar{\sigma} \mu \rho}-h_{\bar{\lambda} \bar{\sigma}} h_{\mu \rho}-H_{\nu \bar{\lambda} \bar{\sigma}} H_{\bar{\nu} \mu \rho}\right) \varpi_{0}^{\rho} \wedge \varpi_{\sigma}^{0} .
\end{aligned}
$$

where $T_{\beta \gamma}^{\alpha}$ and $R_{\beta \gamma \bar{\delta}}^{\alpha}$ are the complex functions defined in (5.12) and (5.13).

We call the equations (5.29) and (5.33) - (5.36) the structure equations of the non-linear Hermitian connection of $(M, J, F)$.

Proof. (5.29) follows from the defining equations of the algebraic vector subspaces. To check (5.30) - (5.32), one has only to use the definitions, Lemma 5.3 and Proposition 5.5 (1). The structure equations (5.33) - (5.36) are proved by evaluating both sides on all possible pairs of vector fields of the absolute parallelism (4.3) and checking that both sides give the same result. This can done directly by using Propositions 5.4, 5.5, 5.6 and formulae (5.31) and (5.32).

Consider the following 2 -forms on $U_{F}(M)$ with values in $\mathfrak{g l}_{\mathfrak{n}}(\mathbb{C}) \oplus \mathbb{C}^{n}$ :

$$
\begin{aligned}
& \Theta=\sum_{\alpha=0}^{n-1} e_{\alpha} \otimes \Theta^{\alpha}, \quad \Sigma=\sum_{\alpha=0}^{n-1} e_{\alpha} \otimes \Sigma^{\alpha}, \quad \Omega=\sum_{\alpha, \beta=0}^{n-1} E_{\alpha}^{\beta} \otimes \Omega_{\beta}^{\alpha}, \\
& \Pi=\sum_{\lambda=1}^{n-1} E_{\lambda}^{0} \otimes \Pi_{0}^{\lambda}+\sum_{\mu=1}^{n-1} E_{0}^{\mu} \otimes \Pi_{\mu}^{0}+\sum_{\lambda, \mu=1}^{n-1} E_{\lambda}^{\mu} \otimes \Pi_{\mu}^{\lambda}, \quad \Phi=\sum_{\lambda, \mu=1}^{n-1} E_{\lambda}^{\mu} \otimes \Phi_{\mu}^{\lambda} .
\end{aligned}
$$

We call $\Theta$ the (pure) torsion form and $\Sigma$ the Finsler torsion form. The 2-form $\Omega$ is called the (pure) curvature form; finally we name $\Pi$ and $\Phi$ oblique Finsler curvature and vertical Finsler curvature, respectively.

The Finsler curvature and torsion forms are 0 if the Finsler metric is associated with an Hermitian metric. The following Proposition gives an important criterion to see when this occurs.

Proposition 5.9. A complex Finsler metric $F$ is associated with an Hermitian metric $g$ if and only if the component $\Sigma^{0}$ of the Finsler torsion form vanishes identically.

In this case, $\Sigma=0, \Pi=0$ and $\Phi=0$ and $\Theta$ and $\Omega$ coincide with the torsion form and the curvature forms of the linear Hermitian connection of $(M, J, g)$, respectively.

Proof. From definitions, $\Sigma^{0}$ vanishes if and only if for any $x \in M$, any $0 \neq v \in$ $T_{x} M$ and any two trivially extended vector fields $X, Y \in T\left(T_{x} M\right), \mathrm{h}\left(X^{10}, Y^{10}\right)=0$. By Lemma 2.5, this occurs if and only if $F^{2}$ is associated to an Hermitian metric $h$. The other part of the claim follows immediately from the identity between $U_{F}(M)$ and the unitary frame bundle corresponding to the Hermitian metric associated with $F$.

Taking the exterior differential of both sides of the structure equations, one can obtain several identities that must be satisfied by the structural functions and by the torsion and curvature forms. Some of them are given in the following Proposition. 
When $F$ is associated with an Hermitian metric, they reduce to the usual symmetry identities and to the Bianchi identities of the torsion and curvature of a linear Hermitian connection.

Proposition 5.10. Let $\Theta$ and $\Omega$ the pure curvature forms of a complex Finsler manifold $(M, J, F)$. Then the components $\Theta^{\alpha}=T_{\beta \gamma}^{\alpha} \theta^{\beta} \wedge \theta^{\gamma}$ and $\Omega_{\beta}^{\alpha}=R_{\beta \gamma \bar{\delta}}^{\alpha} \theta^{\gamma} \wedge \theta^{\bar{\delta}}$ verify the following identities:

$$
\Omega_{\beta}^{\alpha}=-\overline{\Omega_{\alpha}^{\beta}}
$$

(First Bianchi Identities)

$$
\begin{aligned}
& \hat{e}_{\beta}\left(T_{\gamma \delta}^{\alpha}\right)+\hat{e}_{\gamma}\left(T_{\delta \beta}^{\alpha}\right)+\hat{e}_{\delta}\left(T_{\beta \gamma}^{\alpha}\right)+T_{\varepsilon \beta}^{\alpha} T_{\gamma \delta}^{\varepsilon}+T_{\varepsilon \delta}^{\alpha} T_{\beta \gamma}^{\varepsilon}+T_{\varepsilon \gamma}^{\alpha} T_{\delta \beta}^{\varepsilon}=0 \\
& R_{\beta \gamma \bar{\delta}}^{\alpha}-R_{\gamma \beta \bar{\delta}}^{\alpha}-\hat{e}_{\bar{\delta}}\left(T_{\beta \gamma}^{\alpha}\right)-H_{\bar{\alpha} \lambda \beta} R_{0 \gamma \bar{\delta}}^{\lambda}+H_{\bar{\alpha} \lambda \gamma} R_{0 \beta \bar{\delta}}^{\lambda}=0
\end{aligned}
$$

(Second Bianchi Identities)

$$
\begin{aligned}
& \hat{e}_{\gamma}\left(R_{\beta \delta \bar{\varepsilon}}^{\alpha}\right)-\hat{e}_{\delta}\left(R_{\beta \gamma \bar{\varepsilon}}^{\alpha}\right)+R_{\beta \zeta \bar{\varepsilon}}^{\alpha} T_{\gamma \delta}^{\zeta}+\hat{e}_{\gamma}\left(H_{\bar{\alpha} \beta \bar{\lambda}}\right) R_{\lambda \delta \bar{\varepsilon}}^{0}-\hat{e}_{\delta}\left(H_{\bar{\alpha} \beta \bar{\lambda}}\right) R_{\lambda \gamma \bar{\varepsilon}}^{0}=0 \\
& \hat{e}_{\bar{\delta}}\left(R_{\beta \gamma \bar{\varepsilon}}^{\alpha}\right)-\hat{e}_{\bar{\varepsilon}}\left(R_{\beta \gamma \bar{\delta}}^{\alpha}\right)-R_{\beta \gamma \bar{\zeta}}^{\alpha} \overline{T_{\delta \varepsilon}}-\hat{e}_{\bar{\varepsilon}}\left(H_{\bar{\alpha} \beta \lambda}\right) R_{0 \gamma \bar{\delta}}^{\lambda}+\hat{e}_{\bar{\delta}}\left(H_{\bar{\alpha} \beta \lambda}\right) R_{0 \gamma \bar{\varepsilon}}^{\lambda}=0
\end{aligned}
$$

Proof. (1) The identity (5.30) implies that $d \varpi_{\beta}^{\alpha}=-\overline{d \varpi_{\alpha}^{\beta}}$. Then (5.40) follows directly from this and the structure equations.

Now, consider the exterior differential of both sides of the structure equations (5.33) - (5.36). It can be easily checked that the 3 -form

$$
\left(\Omega_{\beta}^{\alpha}-H_{\bar{\alpha} \lambda \beta} \Omega_{0}^{\lambda}\right) \wedge \theta^{\beta}-d \Theta^{\alpha}, \quad d \Omega_{0}^{0}, \quad d \Omega_{0}^{\lambda}+d \Pi_{0}^{\lambda}, \quad d \Omega_{\lambda}^{0}+d \Pi_{\lambda}^{0}, \quad d \Omega_{\mu}^{\lambda}+d \Pi_{\mu}^{\lambda}
$$

vanish identically on any three vectors of horizontal distribution $\mathcal{H}$. This implies that the components of these 3 -forms with respect to the forms

$$
\theta^{\beta} \wedge \theta^{\gamma} \wedge \theta^{\delta}, \theta^{\beta} \wedge \theta^{\gamma} \wedge \theta^{\bar{\delta}}, \theta^{\beta} \wedge \theta^{\bar{\gamma}} \wedge \theta^{\bar{\delta}}, \theta^{\bar{\beta}} \wedge \theta^{\bar{\gamma}} \wedge \theta^{\bar{\delta}}
$$

have to vanish identically. Such components are exactly the left hand sides of (5.41) - (5.44).

Remark 5.11. The exterior differentiation of the structure equations give several other identities for the torsion and the curvature, which are not listed in Proposition 5.10 .

When the Finsler metric is associated with an Hermitian metric, those identities express only the property that $T_{\beta \gamma}^{\alpha}(u)$ and $R_{\beta \gamma \bar{\delta}}^{\alpha}(u)$ depend on the frame $u=\left\{e_{i}\right\}$ as the components of some suitable tensor fields on $M$. But when the Finsler metric $F$ is not associated with an Hermitian metric, these identities give new (and somehow unexpected) relations between $\Theta, \Omega$ and the Finsler torsion and curvatures $\Sigma, \Pi$ and $\Phi$.

One can obtain a complete list of these identities (and avoid several tedious computations) using some symbolic manipulation computer program.

\section{The structure equations of Lempert manifolds.}

6.1. Geodesics of a complex Finsler manifold. We continue to use all conventions given in $\S 5.1, \S 5.2$ and $\S 5.4$. 
Let $\gamma:[a, b] \subset \mathbb{R} \rightarrow M$ be a smooth regular curve, that is a smooth curve such that the tangent vector $\dot{\gamma}_{t}$ is different from 0 for any $t \in[a, b]$. A smooth curve $\tilde{\gamma}:[a, b] \rightarrow U_{F}(M)$ is called lift of $\gamma$ if it satisfies:

a) $\pi \circ \tilde{\gamma}=\gamma$;

b) for any $t \in[a, b]$, the frame $\tilde{\gamma}_{t}=\left\{f_{0}(t), \ldots, f_{2 n-1}(t)\right\} \subset T_{\gamma_{t}}^{10} M$ is so that $f_{0}(t) \in \mathbb{C}^{*} \dot{\gamma}_{t}$.

Notice that a curve $\tilde{\gamma}:[a, b] \rightarrow U_{F}(M)$ is a lift of $\gamma$ if and only if it projects onto $\gamma$ and there exists a smooth map $\varphi:[a, b] \rightarrow \mathbb{R}$ such that for any frame $\tilde{\gamma}_{t}=\left\{f_{i}(t)\right\}$

$$
\dot{\gamma}_{t}=F\left(\dot{\gamma}_{t}\right) e^{\varphi_{t} J} \cdot\left(f_{0}(t)\right)=F\left(\dot{\gamma}_{t}\right)\left(e^{i \varphi_{t}} e_{0}(t)+e^{-i \varphi_{t}} e_{\overline{0}}(t)\right) .
$$

We call length of $\gamma$ and energy of $\gamma$ the integrals $L(\gamma)$ and $E(\gamma)$ defined by

$$
L(\gamma)=\int_{a}^{b} F\left(\dot{\gamma}_{t}\right) d t, \quad E(\gamma)=\int_{a}^{b} F^{2}\left(\dot{\gamma}_{t}\right) d t
$$

Note that if $\tilde{\gamma}$ is any lift of $\gamma$ on $U_{F}(M)$, then

$$
L(\gamma)=\int_{a}^{b} \sqrt{\theta^{0}\left(\dot{\tilde{\gamma}}_{t}\right) \theta^{\overline{0}}\left(\dot{\tilde{\gamma}}_{t}\right)} d t, \quad E(\gamma)=\int_{a}^{b} \theta^{0}\left(\dot{\tilde{\gamma}}_{t}\right) \theta^{\overline{0}}\left(\dot{\tilde{\gamma}}_{t}\right) d t
$$

We recall that a variation with fixed endpoints of $\gamma$ is a smooth map $V:(-\delta, \delta) \times$ $[a, b] \rightarrow M$ such that

(1) $V(0, t)=\gamma_{t}$ for all $t \in[a, b]$;

(2) for any $s \in(-\delta, \delta)$, the curve $\gamma^{(s)}=V(s, *)$ is a regular curve such that $\gamma_{a}^{(s)}=\gamma_{a}$ and $\gamma_{b}^{(s)}=\gamma_{b}$.

Definition 6.1. A regular curve $\gamma:[a, b] \rightarrow M$ is called geodesic of the complex Finsler manifold $(M, J, F)$ if for any variation $V$ with fixed endpoints, the family of curves $\gamma^{(s)}=V(s, *)$ is so that

$$
\left.\frac{d E\left(\gamma^{(s)}\right)}{d t}\right|_{s=0}=0
$$

The equations of Euler-Lagrange for a geodesic of a complex Finsler manifold are given in the following Theorem.

TheOREM 6.2. Let $\gamma:[a, b] \rightarrow M$ be a regular curve.

(1) For any lift $\tilde{\gamma}:[a, b] \rightarrow U_{F}(M)$ of $\gamma$ consider the complex functions $A(\tilde{\gamma})_{t}$, $B(\tilde{\gamma})_{t}$ and $C(\tilde{\gamma})_{t}$ defined by

$$
\begin{gathered}
A(\tilde{\gamma})_{t}=\varpi_{0}^{0}\left(\dot{\tilde{\gamma}}_{t}\right) \theta^{\overline{0}}\left(\dot{\tilde{\gamma}}_{t}\right)-\left.\frac{d \theta^{\overline{0}}\left(\dot{\tilde{\gamma}}_{s}\right)}{d s}\right|_{t}, \\
B(\tilde{\gamma})_{t}=\varpi_{\lambda}^{0}\left(\dot{\tilde{\gamma}}_{t}\right)+\left.T_{\lambda 0}^{0}\right|_{\tilde{\gamma}_{t}} \theta^{0}\left(\dot{\tilde{\gamma}}_{t}\right), \quad C(\tilde{\gamma})_{t}=\varpi_{\bar{\lambda}}^{\overline{0}}\left(\dot{\tilde{\gamma}}_{t}\right)+\left.\overline{T_{\lambda 0}^{0}}\right|_{\tilde{\gamma}_{t}} \theta^{\overline{0}}(\dot{\tilde{\gamma}}) .
\end{gathered}
$$

They vanish identically for one lift of $\gamma$ if and only if they vanish for any lift of $\gamma$.

(2) $\gamma$ is a geodesic if and only if for any lift $\tilde{\gamma}$ and any $t \in[a, b]$

$$
\begin{aligned}
& \frac{d F(\dot{\gamma})}{d t}=0, \quad \varpi_{0}^{0}\left(\dot{\tilde{\gamma}}_{t}\right) \theta^{\overline{0}}\left(\dot{\tilde{\gamma}}_{t}\right)-\left.\frac{d \theta^{0}\left(\dot{\tilde{\gamma}}_{s}\right)}{d s}\right|_{t}=0, \\
& \varpi_{\lambda}^{0}\left(\dot{\tilde{\gamma}}_{t}\right)+\left.T_{\lambda 0}^{0}\right|_{\tilde{\gamma}_{t}} \theta^{0}\left(\dot{\tilde{\gamma}}_{t}\right)=0, \quad \varpi_{\bar{\lambda}}^{\overline{0}}\left(\dot{\tilde{\gamma}}_{t}\right)+\left.\overline{T_{\lambda 0}^{0}}\right|_{\tilde{\gamma}_{t}} \theta^{\overline{0}}\left(\dot{\tilde{\gamma}}_{t}\right)=0 .
\end{aligned}
$$


Proof. (1) Let $\tilde{\gamma}$ and $\tilde{\gamma}^{\prime}$ be two lifts of $\gamma$. Let also $\varphi_{t}$ and $\varphi_{t}^{\prime}$ two real functions so that (6.1) holds for $\tilde{\gamma}$ and $\tilde{\gamma}^{\prime}$, respectively. This means that $\tilde{\gamma}_{t}^{\prime}=\tilde{\gamma}_{t} \circ\left(e^{\left(\varphi_{t}-\varphi_{t}^{\prime}\right) J}\right)$ and that $\dot{\tilde{\gamma}}_{t}^{\prime}=\left(R_{e^{\left(\varphi_{t}-\varphi_{t}^{\prime}\right) J}}\right)_{*}\left(\dot{\tilde{\gamma}}_{t}\right)$ for any $t$. From the invariance properties of $\theta, \omega$ and of the torsion 2-forms $\Theta^{\alpha}$ under the action of $T^{1}$, it follows immediately that $A\left(\tilde{\gamma}^{\prime}\right)=e^{\sqrt{-1}\left(\varphi_{t}^{\prime}-\varphi_{t}\right)} A(\tilde{\gamma}), B\left(\tilde{\gamma}^{\prime}\right)=e^{\sqrt{-1}\left(\varphi_{t}-\varphi_{t}^{\prime}\right)} B(\tilde{\gamma})$ and $C\left(\tilde{\gamma}^{\prime}\right)=e^{\sqrt{-1}\left(\varphi_{t}^{\prime}-\varphi_{t}\right)} C(\tilde{\gamma})$.

(2) Consider a variation $V:(-\delta, \delta) \times[a, b] \rightarrow M$ of $\gamma$ with fixed endpoints and let $\tilde{V}:(-\delta, \delta) \times[a, b] \rightarrow U_{F}(M)$ be a smooth map such that for any $s \in(-\delta, \delta)$, the curve $\tilde{\gamma}^{(s)}=\tilde{V}(s, *)$ is a lift of the curve $\gamma^{(s)}=V(s, *)$.

Let also $X$ and $Y$ the vector fields, which are tangent to $\tilde{V}((-\delta, \delta) \times[a, b]) \subset$ $U_{F}(M)$, defined by

$$
X=\tilde{V}_{*}\left(\frac{\partial}{\partial t}\right), \quad Y=\tilde{V}_{*}\left(\frac{\partial}{\partial t}\right) .
$$

Note that $[X, Y]=\tilde{V}_{*}\left(\left[\frac{\partial}{\partial t}, \frac{\partial}{\partial s}\right]\right)=0$.

From definitions and the commuting property of $X$ and $Y$ we get that

$$
\begin{aligned}
& \left.\frac{d E\left(\gamma^{(s)}\right)}{d s}\right|_{s=0}=\left.\int_{a}^{b} Y\left(\theta^{0}(X) \theta^{\overline{0}}(X)\right)\right|_{\tilde{\gamma}_{t}} d t=\int_{a}^{b}\left[d \theta^{0}(Y, X) \theta^{\overline{0}}(X)\right. \\
& \left.+\theta^{0}(X) d \theta^{\overline{0}}(Y, X)+X\left(\theta^{0}(Y)\right) \theta^{\overline{0}}(X)+\theta^{0}(X) X\left(\theta^{\overline{0}}(Y)\right)\right]\left.\right|_{\tilde{\gamma}_{t}} d t \\
& =\int_{a}^{b}\left[d \theta^{0}(Y, X) \theta^{\overline{0}}(X)+\theta^{0}(X) d \theta^{\overline{0}}(Y, X)-\theta^{0}(Y) X\left(\theta^{\overline{0}}(X)\right)-\right. \\
& \left.-\theta^{\overline{0}}(Y) X\left(\theta^{0}(X)\right)\right]\left.\right|_{\tilde{\gamma}_{t}} d t+\left.\int_{a}^{b} X\left(\theta^{0}(Y) \theta^{\overline{0}}(X)+\theta^{0}(X) \theta^{\overline{0}}(Y)\right)\right|_{\tilde{\gamma}_{t}} d t .
\end{aligned}
$$

Now,

(6.8)

$$
\left.\int_{a}^{b} X\left(\theta^{0}(Y) \theta^{\overline{0}}(X)+\theta^{0}(X) \theta^{\overline{0}}(Y)\right)\right|_{\tilde{\gamma}_{t}} d t=\left.\left(\theta^{0}(Y) \theta^{\overline{0}}(X)+\theta^{0}(X) \theta^{\overline{0}}(Y)\right)\right|_{\tilde{\gamma}_{a}} ^{\tilde{\gamma}_{b}}=0
$$

because $\theta^{0}(Y)_{\tilde{\gamma}_{a}}=\theta^{0}(Y)_{\tilde{\gamma}_{b}}=0$ since $V$ is a variation with fixed endpoints.

Using (6.8), the fact that $\theta^{\alpha}(X)=\delta_{0}^{\alpha} \theta^{0}(X)$ and the structure equations (5.29) and (5.33), we get

$$
\begin{aligned}
\left.(6.9) \frac{d E\left(\gamma^{(s)}\right)}{d s}\right|_{s=0}= & \int_{a}^{b}\left\{\left[-\left(\varpi_{\alpha}^{0} \wedge \theta^{\alpha}\right)(Y, X)+\Theta^{0}(Y, X)+\Sigma^{0}(Y, X)\right] \theta^{\overline{0}}(X)\right. \\
& +\theta^{0}(X)\left[-\left(\omega_{\bar{\alpha}}^{\overline{0}} \wedge \theta^{\bar{\alpha}}\right)(Y, X)+\Theta^{\overline{0}}(Y, X)+\Sigma^{\overline{0}}(Y, X)\right] \\
& \left.-\theta^{0}(Y) X\left(\theta^{\overline{0}}(X)\right)-\theta^{\overline{0}}(Y) X\left(\theta^{0}(X)\right)\right\}_{\tilde{\gamma}_{t}} d t \\
= & \int_{a}^{b}\left\{\theta^{0}(X) \theta^{\overline{0}}(X)\right\}_{\tilde{\gamma}_{t}}\left\{\theta ^ { 0 } ( Y ) \left[\frac{\varpi_{0}^{0}(X)}{\theta^{0}(X)}-\frac{X\left(\theta^{\overline{0}}(X)\right)}{\left.\theta^{0}(X) \theta^{\overline{0}}(X)\right]}\right.\right. \\
& +\theta^{\overline{0}}(Y)\left[\frac{\varpi_{\overline{0}}^{\overline{0}}(X)}{\theta^{\overline{0}}(X)}-\frac{X\left(\theta^{0}(X)\right)}{\theta^{0}(X) \theta^{\overline{0}}(X)}\right]+\theta^{\lambda}(Y)\left[\frac{\varpi_{\lambda}^{0}(X)}{\theta^{0}(X)}+T_{\lambda 0}^{0}\right] \\
& \left.+\theta^{\bar{\lambda}}(Y)\left[\frac{\varpi_{\bar{\lambda}}^{\overline{0}}(X)}{\theta^{\overline{0}}(X)}+T_{\bar{\lambda} \overline{0}}^{\overline{0}}\right]\right\}_{\tilde{\gamma}_{t}} d t .
\end{aligned}
$$


Hence $\gamma$ is a geodesic if and only if equation (6.6) and the following equations (6.10) are identically satisfied:

$$
\varpi_{0}^{0}(X) \theta^{\overline{0}}(X)-\left.X\left(\theta^{\overline{0}}(X)\right)\right|_{\tilde{\gamma}_{t}}=0, \quad \varpi_{\overline{0}}^{\overline{0}}(X) \theta^{0}(X)-\left.X\left(\theta^{0}(X)\right)\right|_{\tilde{\gamma}_{t}}=0 .
$$

Since $\varpi_{0}^{0}+\varpi_{\overline{0}}^{\overline{0}}=0$, multiplying the first and the second equation by $\theta^{0}(X)$ and $\theta^{\overline{0}}(X)$, respectively, and then adding them together we get:

$$
\frac{d\left(\theta^{0}(X) \theta^{\overline{0}}(X)\right)}{d t}=\frac{d F^{2}(\dot{\gamma})}{d t}=0 .
$$

This shows that the equations (6.10) are equivalent to the equations (6.5) and it concludes the proof.

In analogy with what happens in Riemannian geometry, it is not hard to realize that a regular curve is critical w.r.t. the length functional if and only if, up to a parameterization, it is a geodesic.

6.2. Complex geodesics, E-manifolds and Lempert manifolds. Let $\left(N, J_{N}\right)$ be a complex manifold of dimension $\operatorname{dim}_{\mathbb{C}} N \leq \operatorname{dim} M=n$ and let $\imath: N \rightarrow M$ be a holomorphic embedding. In analogy with the Riemannian and Hermitian settings, an holomorphic embedding is said to be totally geodesic whenever any geodesic $\gamma$ : $[a, b] \rightarrow N$ of the induced Finsler metric $F_{N}=F \circ \imath_{*}$ is embedded as a geodesic $\gamma^{\prime}=\imath \circ \gamma$ of $M$.

We give here the concepts of complex geodesics and complex pre-geodesics. Note that our definition of complex geodesics coincides with that of segments of complex geodesics given in [AP]. They are strongly related (but different) with the complex geodesics as defined by Vesentini in [Ve] (see remarks in [AP], p. 129).

Definition 6.3. A complex pre-geodesic of a complex Finsler manifold $(M, J, F)$ is a totally geodesic holomorphic embedding $\imath: \Gamma \rightarrow M$ of a simply connected complex curve $\left(\Gamma, J_{o}\right)$.

A complex geodesic is a complex pre-geodesic $\imath: \Gamma \rightarrow M$ such that the Kähler metric induced on $\Gamma$ by $M$ has constant holomorphic sectional curvature.

A complex Finsler manifold $(M, J, F)$ is called $E$-manifold if

i) for any $x \in M$ and any vector $v \in T_{x} M$ there exists a complex geodesic $\imath: \Gamma \rightarrow M$ passing through $x$ and tangent to $v$;

ii) all complex geodesics have the same holomorphic sectional curvature.

As we mentioned in the Introduction, the examples of E-manifolds we are mainly interested in are the Lempert manifolds (see Definition 1.1): they are E-manifolds with complex geodesics of holomorphic sectional curvature -4 . Other interesting examples of complete E-manifolds, with complex geodesics with non-negative holomorphic sectional curvature, are given by the classification of non-negatively curved Kahler-Finsler manifolds given by Abate and Patrizio in [AP1].

The goal of this subsection is to give some properties on the torsion and the curvature, which gives a complete characterization of the E-manifolds. In the following Theorem 6.7, we will show that the E-manifolds are exactly the complex Finsler manifolds, which are geodetically torsion-free and with constant holomorphic sectional curvature (see Definition 6.4 below).

Notice that what we call geodetically torsion-free Finsler manifolds coincide with the manifolds that Abate and Patrizio christened weakly-Kähler Finsler manifolds (see $[\mathrm{AP}])$. 
We begin with the equations of complex pre-geodesics and the complex geodesics of a complex Finsler manifold.

Let $\imath: \Gamma \rightarrow M$ be a holomorphic embedding of a complex curve $\Gamma$ and let $F_{\Gamma}=F \circ \imath_{*}$ the induced Finsler metric on $\Gamma$. We call adapted unitary frame of $(\Gamma, \imath)$ any frame $u=\left\{f_{0}, \ldots, f_{2 n-1}\right\} \in \pi^{-1}(\imath(\Gamma)) \subset U_{F}(M)$ with $f_{0}$ tangent to $\imath(\Gamma)$. We denote by $U_{\imath}(\Gamma)$ the bundle of adapted unitary frames. It is immediate to realize that $U_{\imath}(\Gamma) / U_{n-1}=U_{F_{\Gamma}}(\Gamma)$.

Let us denote by $\tilde{\imath}: U_{\imath}(\Gamma) \rightarrow U_{F}(M)$ the natural immersion map. Then we have the following commutative diagram.

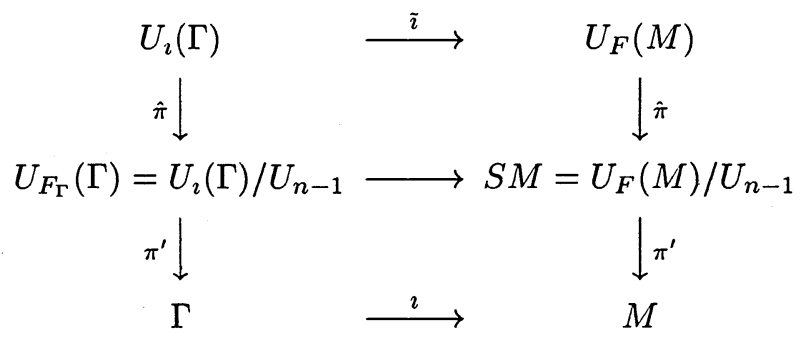

Let us also define on $U_{\imath}(\Gamma)$ the following 1-forms

$$
\vartheta^{\alpha}=\tilde{\imath}^{*} \theta^{\alpha}, \quad \pi_{\beta}^{\alpha}=\tilde{\imath}^{*} \varpi_{\beta}^{\alpha}
$$

If we denote by $\theta_{\Gamma}^{0}$ and $\theta_{\Gamma}^{\overline{0}}$ the holomorphic components of the tautological 1-form of $U_{F_{\Gamma}}(\Gamma)$, then

$$
\vartheta^{0} \equiv \hat{\pi}^{*} \theta_{\Gamma}^{0}, \quad \vartheta^{\lambda} \equiv 0
$$

LEMmA 6.4. Let $\imath: \Gamma \rightarrow M$ be an holomorphic embedding of a complex curve $\Gamma$ in $(M, J, F)$ and let $\varpi_{\beta}^{\alpha}$ the components of the Hermitian connection of the induced metric $F_{\Gamma}=F \circ \imath_{*}$ on $\Gamma$. The embedding is totally geodesic if and only if for any $\lambda=1, \ldots, n-1$ :

(1) $\pi_{0}^{0}=\hat{\pi}^{*}{\underset{\Gamma}{0}}_{0}^{0}$

(2) $\pi_{0}^{\lambda} \equiv 0$ and $\pi_{\lambda}^{0} \equiv 0$;

(3) $T_{\lambda 0}^{0} \circ \tilde{\imath} \equiv 0$.

In particular if $\imath$ is a totally geodesic holomorphic embedding, then for any $u \in U_{F_{\Gamma}}(\Gamma)$ and any $\tilde{u}$ of $\hat{\pi}^{-1}(u) \in U_{\imath}(\Gamma),\left.R_{\lambda 0 \overline{0}}^{0}\right|_{\tilde{i}(u)}=0$ and the holomorphic sectional curvature $\left.c\right|_{u}$ of $F_{\Gamma}$ is equal to

$$
\left.c\right|_{u}=R_{00 \overline{0}}^{0} \mid \tilde{\imath}(u)
$$

Proof. Let $\gamma:[a, b] \rightarrow \Gamma$ be a regular curve in $\Gamma$ and let $\tilde{\gamma}:[a, b] \rightarrow U_{\imath}(\Gamma) \subset$ $U_{F}(M)$ be a lift of the curve $\gamma_{\imath}=\imath \circ \gamma$. By (6.16) and Theorem 6.2, $\gamma$ is a geodesic for the induced metric if and only if

$$
\frac{d F_{\Gamma}(\dot{\gamma})}{d t}=\frac{d F\left(\dot{\gamma}_{\imath}\right)}{d t}=0, \quad \vartheta^{\overline{0}}(\dot{\tilde{\gamma}})\left(\hat{\pi}^{*} \varpi_{\Gamma}^{0}\right)\left(\dot{\tilde{\gamma}}_{t}\right)-\left.\frac{d \vartheta^{\overline{0}}\left(\dot{\gamma}_{s}\right)}{d s}\right|_{t}=0 .
$$

Using again Theorem $6.2, \gamma_{2}$ is a geodesic for the Finsler metric of $M$ if and only if $\tilde{\gamma}$ 
verifies also

$$
\begin{aligned}
& \vartheta^{\overline{0}}\left(\dot{\tilde{\gamma}}_{t}\right) \pi_{0}^{0}\left(\dot{\tilde{\gamma}}_{t}\right)-\left.\frac{d \vartheta^{\overline{0}}\left(\dot{\tilde{\gamma}}_{s}\right)}{d s}\right|_{t}=0 \\
& \pi_{\lambda}^{0}\left(\dot{\tilde{\gamma}}_{t}\right)+\left.T_{\lambda 0}^{0}\right|_{\tilde{\gamma}_{t}} \vartheta^{0}(\dot{\tilde{\gamma}})=0, \quad \pi_{\bar{\lambda}}^{\overline{0}}\left(\dot{\tilde{\gamma}}_{t}\right)+\left.\overline{T_{\lambda 0}^{0}}\right|_{\tilde{\gamma}_{t}} \vartheta^{\overline{0}}(\dot{\tilde{\gamma}})=0 .
\end{aligned}
$$

Therefore the embedding is totally geodesic if and only if (1) holds and

$$
\left.\pi_{\lambda}^{0}\right|_{u}=-\left.T_{\lambda 0}^{0}\right|_{u} \vartheta_{u}^{0},\left.\quad \pi_{\bar{\lambda}}^{\overline{0}}\right|_{u}=-\left.\overline{T_{\lambda 0}^{0}}\right|_{u} \vartheta_{u}^{\overline{0}}
$$

for any $u \in U_{\imath}(\Gamma)$. On the other hand, by the structure equations (5.29) and (5.30) and by (6.16)

$$
0=d \vartheta^{\lambda}=-\pi_{0}^{\lambda} \wedge \vartheta^{0}+\imath^{*} \Theta^{\lambda}=\pi_{\bar{\lambda}}^{\overline{0}} \wedge \vartheta^{0}
$$

From (6.20) and (6.21) it follows that

$$
\left(\overline{T_{\lambda 0}^{0}} \circ \tilde{\imath}\right) \vartheta^{\overline{0}} \wedge \vartheta^{0} \equiv 0
$$

which implies that $\overline{T_{\lambda 0}^{0}} \circ \tilde{\imath} \equiv 0$ since $\vartheta^{0} \wedge \vartheta^{\overline{0}}=\hat{\pi}^{*}\left(\theta_{\Gamma}^{0} \wedge \theta_{\Gamma}^{\overline{0}}\right) \neq 0$. From this, (2) and (3) are immediate.

The last claims follows from (1), (2) and the structure equations of $U_{F_{\Gamma}}(\Gamma)$.

We can now give the characterization of E-manifolds. Let us first introduce some terminology.

Definition 6.5. We say that a complex Finsler manifold $(M, J, F)$ is called geodetically torsion-free if the 2 -form $\Theta^{0}$ is of the following form

$$
\Theta^{0}=\frac{1}{2} T_{\lambda \mu}^{0} \theta^{\lambda} \wedge \theta^{\mu}
$$

(i.e. the complex functions $T_{\lambda 0}^{0}$ vanish identically).

$(M, J, F)$ is called with constant holomorphic sectional curvature if there exists a constant $c$ so that the 2 -forms $\Omega_{0}^{0}$ and $\Omega_{\lambda}^{0}$ are of the form

$$
\begin{aligned}
& \Omega_{0}^{0}=c \theta^{0} \wedge \theta^{\overline{0}}+R_{0 \rho \bar{\sigma}}^{0} \theta^{\rho} \wedge \theta^{\bar{\sigma}}+R_{0 \rho \overline{0}}^{0} \theta^{\rho} \wedge \theta^{\overline{0}}+R_{00 \bar{\sigma}}^{0} \theta^{0} \wedge \theta^{\bar{\sigma}} \\
& \Omega_{0}^{\lambda}=R_{0 \rho \bar{\sigma}}^{\lambda} \theta^{\rho} \wedge \theta^{\bar{\sigma}}+R_{0 \rho \overline{0}}^{\lambda} \theta^{\rho} \wedge \theta^{\overline{0}}+R_{00 \bar{\sigma}}^{\lambda} \theta^{0} \wedge \theta^{\bar{\sigma}} \\
& \Omega_{\lambda}^{0}=R_{\lambda \rho \bar{\sigma}}^{0} \theta^{\rho} \wedge \theta^{\bar{\sigma}}+R_{\lambda \rho \overline{0}}^{0} \theta^{\rho} \wedge \theta^{\overline{0}}+R_{\lambda 0 \bar{\sigma}}^{0} \theta^{0} \wedge \theta^{\bar{\sigma}}
\end{aligned}
$$

(i.e. $R_{00 \overline{0}}^{0} \equiv c$ and $R_{00 \overline{0}}^{\lambda} \equiv R_{\lambda 0 \overline{0}}^{0} \equiv 0$ ).

If $M$ has constant holomorphic sectional curvature, the constant $c$ is called the holomorphic sectional curvature of $M$.

Remark 6.6. Assume that $F$ is associated with an Hermitian metric $g$. In this case, using the fact that the functions $T_{\beta \gamma}^{\alpha}(u)$ depends on the frame $u$ as the components of a tensor of type $(1,2)$, it can be inferred that $F$ is geodetically torsion-free if and only if $g$ is torsion free and hence Kähler. With the same arguments, it can be shown that $F$ is of constant holomorphic sectional curvature if and only if the Hermitian metric $g$ is of constant holomorphic sectional curvature.

Notice that the notion of manifold with constant holomorphic sectional curvature is not the same as manifold with constant holomorphic curvature as defined by Abate and Patrizio in [AP], although the two notions turn out to be equivalent in 
the Hermitian case. In [AP], the conditions $R_{00 \overline{0}}^{\lambda} \equiv R_{\lambda 0 \overline{0}}^{0} \equiv 0$ are not included in the definition of manifolds with constant holomorphic curvature.

Here is the characterization we were looking for.

TheOREM 6.7. Let $(M, J, F)$ be a complex Finsler manifold.

i) There exists a complex pre-geodesic through any point $x \in M$ and tangent to any vector $v \in T_{x} M$ if and only $M$ is geodetically torsion-free.

ii) $(M, J, F)$ is an E-manifold if and only if it is geodetically torsion-free and with constant holomorphic sectional curvature.

Proof. (i) The necessity follows immediately from Lemma 6.5. Suppose now that $(M, J, F)$ is geodetically torsion-free and consider the distribution $\mathcal{C}$ on $U_{F}(M)$ given by all vectors $X \in T U_{F}(M)$ such that

$$
\theta^{\lambda}(X)=0, \quad \theta^{\bar{\lambda}}(X)=0, \quad \varpi_{0}^{\lambda}(X)=0, \quad \varpi_{\lambda}^{0}(X)=0
$$

for $\lambda=1, \ldots, n-1$. Using the structure equations, one can check that the equations (6.24) define an integrable distribution whose integral leaves of maximal dimension project onto holomorphic curves in $(M, J)$. Moreover, if $S \subset U_{F}(M)$ is an integral leaf of $\mathcal{C}$ with corresponding holomorphic curve $\Gamma=\pi(S) \subset M$ and if $\imath: \Gamma \rightarrow M$ is the standard immersion of $\Gamma$, then $S$ is equal to the adapted frame bundle $U_{2}(\Gamma)$ and the immersion $\imath: \Gamma \rightarrow M$ is a totally geodesic isometric embedding. Since there exists an integral leaf of $\mathcal{C}$ for any frame $u \in U_{F}(M)$, this concludes the proof of (i).

The proof of (ii) is analogous.

Remark 6.8. Equivalent characterizations of the E-manifolds can be also found in $[\mathrm{Fa}],[\mathrm{Pa}]$ and $[\mathrm{AP}]$.

6.3. The torsion and curvature of an E-manifold. In the following last Theorem 6.7, we prove that the torsion and the curvature of an E-manifold are uniquely determined by the Finsler torsion and the Finsler curvatures. This implies that in order to have a complete set of invariants for an E-manifold, it suffices to consider the structure functions described in Corollary $5.7 \mathrm{i}$ ), ii) and iii). We also give the explicit formulae for some components of the torsion and the curvature and an application of these formulae, which gives a short proof of an Abate and Patrizio's result on Kähler-Finsler manifolds with positive sectional curvature (Theorem 1.1 in [AP1]).

For the notation and the indexing conventions, see $\S 5.1$ and $\S 5.2$.

THEOREM 6.9. Let $(M, J, F)$ be an E-manifold with constant holomorphic sectional curvature $c$. Then:

(1) the torsion and the curvature of the non-linear Hermitian connection of $M$ are uniquely determined by the structure functions $h_{\lambda \mu}, H_{\lambda \mu \bar{\nu}}, P_{0 \bar{\mu} \gamma}^{\lambda}=\hat{e}_{\gamma}\left(h_{\bar{\lambda} \bar{\mu}}\right)$ and $Q_{\sigma \lambda \bar{\mu}}^{\rho}=H_{\bar{\mu} \bar{\rho} \sigma \lambda}-h_{\bar{\mu} \bar{\rho}} h_{\sigma \lambda}-H_{\nu \bar{\mu} \bar{\rho}} H_{\sigma \lambda \bar{\nu}}$ and their first order derivatives; in particular,

$$
\begin{aligned}
& R_{00 \overline{0}}^{0}=c, \quad R_{\lambda 0 \overline{0}}^{0}=R_{00 \overline{0}}^{\lambda}=R_{0 \lambda \overline{0}}^{0}=R_{00 \bar{\lambda}}^{0}=0, \quad R_{\lambda \mu \overline{0}}^{0}=c h_{\lambda \mu}, \quad R_{00 \bar{\mu}}^{\lambda}=c h_{\bar{\lambda} \bar{\mu}}, \\
& R_{\lambda 0 \bar{\mu}}^{0}=\frac{c}{2}\left(\delta_{\lambda \mu}+h_{\lambda \rho} h_{\bar{\rho} \bar{\mu}}\right), \quad R_{0 \mu \overline{0}}^{\lambda}=\frac{c}{2}\left(\delta_{\lambda \mu}+h_{\bar{\lambda} \bar{\rho}} h_{\rho \mu}\right), \quad R_{0 \lambda \bar{\mu}}^{0}=\frac{c}{2}\left(\delta_{\lambda \mu}-h_{\mu \rho} h_{\bar{\rho} \bar{\lambda}}\right), \\
& R_{\mu 0 \overline{0}}^{\lambda}=\frac{c}{2}\left(\delta_{\lambda \mu}-h_{\nu \mu} h_{\bar{\nu} \bar{\lambda}}\right)-\hat{e}_{\overline{0}}\left(h_{\mu \nu}\right) \hat{e}_{0}\left(h_{\bar{\lambda} \bar{\nu}}\right) .
\end{aligned}
$$

(2) if $c \neq 0$, the 0 -th component of the torsion $\Theta^{0}=T_{\beta \gamma}^{0} \theta^{\beta} \wedge \theta^{\gamma}$ vanishes identically and the whole set of components of the torsion is given by the following expres- 
sions:

$$
T_{\beta \gamma}^{0}=0, \quad T_{0 \gamma}^{\beta}=-\hat{e}_{0}\left(h_{\bar{\beta} \bar{\nu}}\right) h_{\nu \gamma}, \quad T_{\beta \gamma}^{\alpha}=\hat{e}_{\gamma}\left(h_{\bar{\alpha} \bar{\nu}}\right) h_{\nu \beta}-\hat{e}_{\beta}\left(h_{\bar{\alpha} \bar{\nu}}\right) h_{\nu \gamma} .
$$

(3) if $c>0$ and the functions $\hat{e}_{0}\left(T_{0 \lambda}^{\lambda}\right)$ vanish identically for any $\lambda=1, \ldots, n-1$, then $F$ is associated with a Kähler metric of constant holomorphic sectional curvature; in particular, if $(M, J, F)$ is also simply connected and complete, then it is biholomorphic to $\mathbb{C} P^{n}$.

Proof. (1) The proof is based on iterated use of the identities (5.36), the Bianchi identities and the Jacobi identities applied to three vector fields $v_{1}, v_{2}$ and $v_{3}$ on $U_{F}(M)$, where $v_{1}$ and $v_{2}$ are of the form $\hat{e}_{\alpha}$ or $\hat{e}_{\bar{\alpha}}$ and $v_{3}$ is a vector field of the form $\tilde{e}_{\lambda}$ or $\tilde{e}_{\bar{\lambda}}$. The arguments are simple and straightforward and we are going to show only how to determine the expressions for the components $R_{\beta \gamma \bar{\delta}}^{\alpha}$ were at least two indices are equal to 0 or $\overline{0}$. The way to determine all other components of the curvature and of the components of the torsion are analogous.

By hypotheses, for any $\lambda=1, \ldots n-1, R_{00 \overline{0}}^{0}=c, R_{\lambda 0 \overline{0}}^{0}=R_{00 \overline{0}}^{\lambda}=0$ and $T_{0 \lambda}^{0}=0$. Then, from the Bianchi identities (5.42) we get

$$
R_{0 \lambda \overline{0}}^{0}-R_{\lambda 0 \overline{0}}^{0}-\hat{e}_{\overline{0}}\left(T_{0 \lambda}^{0}\right)-H_{\overline{0} \mu 0} R_{0 \lambda \overline{0}}^{\mu}+H_{\overline{0} \mu \lambda} R_{00 \overline{0}}^{\mu}=R_{0 \lambda \overline{0}}^{0}-R_{\lambda 0 \overline{0}}^{0}=0 .
$$

On the other hand, by (5.36)

$$
R_{\beta \gamma \bar{\delta}}^{\alpha}=\overline{R_{\alpha \delta \bar{\gamma}}^{\beta}}
$$

From (6.25) and (6.26), we conclude that $R_{00 \bar{\lambda}}^{0}=R_{0 \lambda \overline{0}}^{0}=0$.

Now, using the notation of $\S 5$, by the Jacobi identities we have

$$
\begin{aligned}
& \omega_{0}^{0}\left(\left[\tilde{e}_{\lambda},\left[\hat{e}_{\mu}, \hat{e}_{\overline{0}}\right]\right]\right)+\omega_{0}^{0}\left(\left[\hat{e}_{\mu},\left[\hat{e}_{\overline{0}}, \tilde{e}_{\lambda}\right]\right]\right)+\omega_{0}^{0}\left(\left[\hat{e}_{\overline{0}},\left[\tilde{e}_{\lambda}, \hat{e}_{\mu}\right]\right]\right) \\
= & R_{\lambda \mu \overline{0}}^{0}-\tilde{e}_{\lambda}\left(R_{0 \mu \overline{0}}^{0}\right)-H_{\alpha \lambda \mu} R_{0 \alpha \overline{0}}^{0}=R_{\lambda \mu \overline{0}}^{0}-c h_{\lambda \mu}=0 .
\end{aligned}
$$

From (6.27) and (6.26), it follows also that $R_{00 \bar{\mu}}^{\lambda}=\overline{R_{\lambda \mu \overline{0}}^{0}}=c h_{\bar{\lambda} \bar{\mu}}$.

Let us use again the first Bianchi identities and the Jacobi identities:

$$
\begin{aligned}
& R_{\lambda 0 \bar{\mu}}^{0}-R_{0 \lambda \bar{\mu}}^{0}-\hat{e}_{\bar{\mu}}\left(T_{\lambda 0}^{0}\right)-H_{\overline{0} \nu \lambda} R_{00 \bar{\mu}}^{\nu}+H_{\overline{0} \nu 0} R_{0 \lambda \bar{\mu}}^{\nu} \\
= & R_{\lambda 0 \bar{\mu}}^{0}-R_{0 \lambda \bar{\mu}}^{0}-c h_{\nu \lambda} h_{\bar{\nu} \bar{\mu}}=0 \\
& \omega_{0}^{0}\left(\left[\tilde{e}_{\lambda},\left[\hat{e}_{0}, \hat{e}_{\bar{\mu}}\right]\right]\right)+\omega_{0}^{0}\left(\left[\hat{e}_{0},\left[\hat{e}_{\bar{\mu}}, \tilde{e}_{\lambda}\right]\right]\right)+\omega_{0}^{0}\left(\left[\hat{e}_{\bar{\mu}},\left[\tilde{e}_{\lambda}, \hat{e}_{0}\right]\right]\right) \\
= & -\tilde{e}_{\lambda}\left(R_{00 \bar{\mu}}^{0}\right)+R_{\lambda 0 \bar{\mu}}^{0}-R_{00 \overline{0}}^{0} \delta_{\lambda \mu}+R_{00 \lambda \bar{\mu}}^{0}=R_{\lambda 0 \bar{\mu}}^{0}+R_{0 \lambda \bar{\mu}}^{0}-c \delta_{\lambda \mu}=0 .
\end{aligned}
$$

From (6.28), (6.29) and (6.26), it follows that

$$
R_{\lambda 0 \bar{\mu}}^{0}=\overline{R_{0 \mu \overline{0}}^{\lambda}}=\frac{c}{2}\left(\delta_{\lambda \mu}+h_{\nu \lambda} h_{\bar{\nu} \bar{\mu}}\right), \quad R_{0 \lambda \bar{\mu}}^{0}=\frac{c}{2}\left(\delta_{\lambda \mu}-h_{\nu \lambda} h_{\bar{\nu} \bar{\mu}}\right) .
$$

Using again the Jacobi identities,

$$
\begin{aligned}
& \omega_{\overline{0}}^{\bar{\mu}}\left(\left[\tilde{e}_{\bar{\lambda}},\left[\hat{e}_{0}, \hat{e}_{\overline{0}}\right]\right]\right)+\omega_{\overline{\bar{\mu}}}^{\bar{\mu}}\left(\left[\hat{e}_{0},\left[\hat{e}_{\overline{0}}, \tilde{e}_{\bar{\lambda}}\right]\right]\right)+\omega_{\overline{0}}^{\bar{\mu}}\left(\left[\hat{e}_{\overline{0}},\left[\tilde{e}_{\bar{\lambda}}, \hat{e}_{0}\right]\right]\right) \\
= & R_{\mu 0 \overline{0}}^{\lambda}-c \delta_{\lambda \mu}+\frac{c}{2}\left(\delta_{\lambda \mu}+h_{\nu \mu} h_{\bar{\nu} \bar{\lambda}}\right)+\hat{e}_{\overline{0}}\left(h_{\mu \nu}\right) \hat{e}_{0}\left(h_{\bar{\lambda} \bar{\nu}}\right)=0 .
\end{aligned}
$$

(2) Assume $c \neq 0$. From the Bianchi identity (5.43) and (1),

$$
\hat{e}_{\beta}\left(R_{0 \gamma \overline{0}}^{0}\right)-\hat{e}_{\gamma}\left(R_{0 \beta \overline{0}}^{0}\right)+R_{0 \delta \overline{0}}^{0} T_{\beta \gamma}^{\delta}=c T_{\beta \gamma}^{0}=0,
$$


and this implies $T_{\beta \gamma}^{0}=0$. Then using again the Jacobi identities, by the vanishing of $\sum_{\text {cyclic perm. }} \theta^{0}\left(\left[v_{i},\left[v_{j}, v_{k}\right]\right]\right)=0$ when $v_{1}=\hat{e}_{\alpha}, v_{2}=\hat{e}_{\nu}$ and $v_{3}=\tilde{e}_{\bar{\lambda}}$, one obtains the remaining expressions for $T_{0 \nu}^{\lambda}$ and for $T_{\mu \nu}^{\lambda}$.

(3) From the Bianchi identity (5.36), we have that

$$
R_{0 \lambda \overline{0}}^{\lambda}-R_{\lambda 0 \overline{0}}^{\lambda}-\hat{e}_{\overline{0}}\left(T_{0 \lambda}^{\lambda}\right)=0 .
$$

By the expressions for the curvature components given in (1), this becomes

$$
\begin{aligned}
& \frac{c}{2}\left(1+\sum_{\rho=1}^{n-1}\left|h_{\lambda \rho}\right|^{2}\right)-\frac{c}{2}\left(1-\sum_{\rho=1}^{n-1}\left|h_{\lambda \rho}\right|^{2}\right)+\sum_{\rho=1}^{n-1}\left|\hat{e}_{\overline{0}}\left(h_{\lambda \rho}\right)\right|^{2}-\hat{e}_{\overline{0}}\left(T_{0 \lambda}^{\lambda}\right) \\
= & \sum_{\rho=1}^{n-1}\left(c\left|h_{\lambda \rho}\right|^{2}+\left|\hat{e}_{\overline{0}}\left(h_{\lambda \rho}\right)\right|^{2}\right)-\hat{e}_{\overline{0}}\left(T_{0 \lambda}^{\lambda}\right)=0 .
\end{aligned}
$$

This implies that, if $c>0$ and $\hat{e}_{\overline{0}}\left(T_{0 \lambda}^{\lambda}\right)=0$ for any $\lambda$, then $\hat{e}_{\overline{0}}\left(h_{\lambda \rho}\right)=h_{\lambda \rho}=0$ for any $\lambda$ and $\rho$. Therefore, by Lemma $2.5, F$ is associated with an Hermitian metric $g$, which is geodetically torsion free and with constant holomorphic sectional curvature. By Remark 6.6, we obtain that $(M, J, g)$ is Kähler and locally isometric to $\mathbb{C} P^{n}$. The conclusion follows from standard facts on complex space forms.

Appendix. We recall here the Cartan-Sternberg theorem on the local automorphisms of an absolute parallelism. The theorem was first proved for real analytic vector fields by $\mathrm{E}$. Cartan and in this case it is a corollary of Cartan-Kähler theorem (see e.g. [BCG]). Later it was proved by S. Sternberg for smooth vector fields ([St]).

Before stating the theorem we need some preliminaries.

Let $\sigma=\left\{X_{1}, \ldots, X_{n}\right\}$ be an absolute parallelism on a manifold $N$. The structure functions of $\sigma$ are the smooth functions $c_{j k}^{i}$ defined by

$$
\left[X_{j}, X_{k}\right]=\sum_{i=1}^{n} c_{j k}^{i} X_{i}
$$

Let us also denote by $c_{j k, m_{1} \ldots m_{r}}^{i}$ the smooth functions defined inductively on $r$ as

$$
c_{j k, m_{1}}^{i}=X_{m_{1}}\left(c_{j k}^{i}\right), \quad c_{j k, m_{1} \ldots m_{r}}^{i}=X_{m_{r}}\left(c_{j k, m_{1} \ldots m_{r-1}}^{i}\right) .
$$

Finally, for any integer $\alpha>0$ let $\mathcal{F}^{(\alpha)}$ be the family of smooth functions

$$
\mathcal{F}^{(\alpha)}=\left\{c_{j k}^{i}, c_{j k, m_{1}}^{i}, \ldots, c_{j k, m_{1} \ldots m_{\alpha}}^{i}\right\}
$$

and call $Q_{\alpha}$ the number of functions in the set $\mathcal{F}^{(\alpha)}$. We consider $\mathcal{F}^{(\alpha)}$ as the set of components of a smooth map from $M$ into $\mathbb{R}^{Q_{\alpha}}$. A point $p \in M$ is called a regular point for $\sigma$ if there exists two integers $s$ and $r$ such that $\operatorname{rank} \mathcal{F}^{(s)}=\operatorname{rank} \mathcal{F}^{(s+1)}=r$ at all points of a neighborhood $U_{p}$ of $p$.

If $s$ is the smallest integer such that this occurs, then $s$ and $r$ are called order and rank of the regular point $p$, respectively.

It can be shown that $\operatorname{rank} \mathcal{F}^{(\alpha)}=r$ for all $\alpha \geq s$ and that there exists a system of coordinates $\left\{x_{1}, \ldots, x_{n}\right\}: U_{p} \rightarrow \mathbb{R}^{n}$ such that all maps $\left.\mathcal{F}^{(\alpha)}\right|_{U_{p}}, \alpha>0$, depend only on the first $r$ coordinates $\left\{x_{1}, \ldots, x_{r}\right\}$ (see [St]). Such a system of coordinates is called adapted to the absolute parallelism. 
For any the adapted system of coordinates $\left\{x_{1}, \ldots, x_{n}\right\}$ on a neighborhood $U_{p}$, we call slice of $U_{p}$ any set of the form

$$
S_{\left(c_{1}, \ldots, c_{r}\right)}=\left\{q \in U: x_{1}(q)=c_{1}, \ldots, x_{r}(q)=c_{r}\right\}
$$

for some $\left(c_{1}, \ldots, c_{r}\right) \in \mathbb{R}^{r}$.

Theorem A.1. (Cartan -Sternberg) Let $\sigma=\left\{X_{1}, \ldots, X_{n}\right\}$ be an absolute parallelism on $M$ and let $p, p^{\prime} \in M$ be two regular point of ranks $r_{p}$ and $r_{p^{\prime}}$ and orders $s_{p}$ and $s_{p^{\prime}}$, respectively. Let also $U$ and $U^{\prime}$ be two neighborhoods of $p$ and $p^{\prime}$, respectively, which admit two adapted systems of coordinates $\left\{x_{i}\right\}$ and $\left\{x_{i}^{\prime}\right\}$.

If $q$ is a point of the slice $S_{\left(c_{1}, \ldots, c_{r}\right)} \subset U$ and $q^{\prime}$ is a point of the slice $S_{\left(c_{1}^{\prime}, \ldots, c_{r}^{\prime}\right)} \subset$ $U^{\prime}$, there exists a local diffeomorphism $f: U \rightarrow U^{\prime}$ such that $f(q)=q^{\prime}$ and $f_{*}\left(X_{i}\right)=X_{i}$ for all $i=1, \ldots, n$, if and only if $r_{p}=r_{p^{\prime}}=r, s_{p}=s_{p^{\prime}}=s$ and $\left.\mathcal{F}^{(s)}\right|_{S_{\left(c_{1}, \ldots, c_{r}\right)}} \equiv$ $\left.\mathcal{F}^{(s)}\right|_{S_{\left(c_{1}^{\prime}, \ldots, c_{r}^{\prime}\right)}}$, and the functional dependence of $\left.\mathcal{F}^{(s+1)}\right|_{U}$ on the functions $\left.\mathcal{F}^{(s)}\right|_{U}$ is the same of the functional dependence of $\left.\mathcal{F}^{(s+1)}\right|_{U^{\prime}}$ on the functions $\left.\mathcal{F}^{(s)}\right|_{U^{\prime}}$.

In particular, if $U=U^{\prime}$, there exists a local diffeomorphism $f: U \rightarrow U$ such that $f(q)=q^{\prime}$ and $f_{*}\left(X_{i}\right)=X_{i}$ for all $i=1, \ldots, n$ if and only if $q$ and $q^{\prime}$ belong to the same slice $S_{\left(c_{1}, \ldots, c_{r}\right)}$ for some $\left(c_{1}, \ldots, c_{r}\right) \in \mathbb{R}^{r}$.

\section{REFERENCES}

[AP] M. Abate And G. Patrizio, Finsler Metrics - A Global Approach, Lecture Notes in Mathematics 1591, Springer-Verlag, 1994.

[AP1] M. ABATE AND G. PATRIZIO, Kähler Finsler Manifolds of constant holomorphic curvature, Inter. Jour. of Math., 8:2 (1997), pp. 169-186.

[BC] D. BAO AND S. S. Chern, On a notable connection in Finsler geometry, Trans. Am. Math. Soc., 79 (1955), pp. 378-180.

[BCG] R. L. Bryant, S. S. Chern, R. B. Gardner, H. L. Goldshmidt, and P. A. Griffiths, Exterior Differential Systems, Springer-Verlag, 1991.

[Be] A. Besse, Einstein Manifolds, Springer-Verlag, 1987.

[Bol] A. Borel, Some remarks about Lie groups transitive on spheres and tori, Bull. Am. Math. Soc., 55 (1949), pp. 580-587.

[Bo2] A. Borel, Le plan projectif des octave et les sphères comme espaces homogènes, C. R. Acad. Sc. Paris, 230 (1950), pp. 1378-1380.

[Ch] S. S. ChERN, Local equivalence and euclidean connections in Finsler spaces, Sci. Rep. Nat. Tsing Hua Univ. (Ser. A), 5 (1948), pp. 95-121.

[Ch1] S. S. Chern, On Finsler geometry, C. R. Acad. Sc. Paris, 314 (1992), pp. 757-761.

[Fa] J. J. FARAN, V, Hermitian Finsler metrics and the Kobayashi metric, J. Diff. Geom., 31 (1990), pp. 601-625.

[Le] L. Lempert, La métrique de Kobayashi et la représentation des domaines sur la boule, Bull. Soc. Math. France, 109 (1981), pp. 427-474.

[Le1] L. Lempert, Intrinsic Distances and Holomorphic Retracts, Complex Analysis and Applications '81 (1984), Sofia.

[Le2] L. LEMPERT, Holomorphic invariants, normal forms and the moduli space of convex domains, Ann. of Math., 128 (1988), pp. 43-78.

[Ko] S. Koвayashi, Hyperbolic manifolds and holomorphic mappings, Dekker, New York, 1970.

[Kol] S. КовауаShi, Transformation Groups in Differential Geometry, Springer-Verlag, 1972.

[Ko2] S. Kobayashi, Negative vector bundles and complex Finsler structures, Nagoya Math. J., 57 (1975), pp. 153-166.

[KN] S. Ковayashi and K. Nomizu, Foundations of Differential Geometry I \& II, Interscience Publ., 1963-1969.

[MS] D. Montgomery And H. Samelson, Transformation groups of spheres, Annals of Math., 44 (1943), pp. 457-469. 
[Pa] M.-Y. PANG, Finsler metrics with properties of the Kobayashi metric on convex domains, Publicationes Matemàtiques, 36 (1992), pp. 131-155.

[Sp] A. SPIRo, Chern's orthonormal frame bundle of a Finsler space, to appear on Houston J. Math..

[St] S. Sternberg, Lectures on Differential Geometry, Prentice Hall, Englewood Cliffs, N.J., 1964.

[Ve] E. Vesentini, Complex geodesics, Comp. Math., 44 (1981), pp. 375-394.

[We] S. Webster, Pseudo-hermitian Structures on a Real Hypersurface, J. Diff. Geo., 13 (1978), pp. 25-41.

[We1] S. WEBSTER, On the reflection principle in several complex variables, Proc. Amer. Math. Soc., 71 (1978), pp. 26-28. 